University of Louisville

ThinkIR: The University of Louisville's Institutional Repository

Electronic Theses and Dissertations

$12-2012$

\title{
Regulation of vascular smooth muscle cell phenotype.
}

Joshua Kuimeta Salabei

University of Louisville

Follow this and additional works at: https://ir.library.louisville.edu/etd

\section{Recommended Citation}

Salabei, Joshua Kuimeta, "Regulation of vascular smooth muscle cell phenotype." (2012). Electronic Theses and Dissertations. Paper 1250.

https://doi.org/10.18297/etd/1250

This Doctoral Dissertation is brought to you for free and open access by ThinkIR: The University of Louisville's Institutional Repository. It has been accepted for inclusion in Electronic Theses and Dissertations by an authorized administrator of ThinkIR: The University of Louisville's Institutional Repository. This title appears here courtesy of the author, who has retained all other copyrights. For more information, please contact thinkir@louisville.edu. 


\title{
REGULATION OF VASCULAR SMOOTH MUSCLE CELL PHENOTYPE
}

\author{
By \\ Joshua Kuimeta Salabei \\ B.S, University of Buea, Cameroon, 2004 \\ M.S., University of Nottingham, England, 2007

\begin{abstract}
A Dissertation in partial Fulfillment of the Requirements

for the Degree of

Doctor of Philosophy

Department of Biochemistry and Molecular Biology

University of Louisville,

Louisville, Kentucky

December 2012
\end{abstract} \\ Submitted to the University of Louisville School of Medicine
}




\title{
REGULATION OF VASCULAR SMOOTH MUSCLE CELL PHENOTYPE
}

\author{
By \\ Joshua Kuimeta Salabei \\ B.S., University of Buea, Cameroon, 2004 \\ M.S., University of Nottingham, England, 2007 \\ A Dissertation \\ November 20, 2012 \\ By the following Dissertation Committee: \\ Aruni Bhatnagar, Ph.D. \\ Bradford G. Hill, Ph.D. \\ Steven P. Jones, Ph.D. \\ Russel A. Prough, Ph.D. \\ William Dean, Ph.D.
}




\section{ACKNOWLEDGEMENTS}

This dissertation is dedicated to my Mom, Monica, my Dad, Elias, my wife, Syntyche and my son, Josh-Eithan. It has always been fun surrounded by loved

ones. My parents never made it past elementary education but they labored hard to push all 6 kids through some university education. Their dedication to educate has motivated me to be determined to succeed come what may. I promise to do the same to my kid(s). My wife has been exceptional. She is truly an African queen, putting everything in order at home. She willingly gets up every morning to prepare breakfast, pack my lunch and make sure we have dinner every single day. This is to be greatly appreciated because her caliber is a rarity. I love you and will always do. Big shout out to my brothers, Chris, Emmanuel, Phil, and Henschel and to my sisters Rachel and Pris. We have a doctor in the family! (Not the type that directly helps patients though).

Thanks to Dr. Bhatnagar and Dr. Hill for their mentoring. Thanks for giving me the opportunity to work in your lab. Dr. Bhatnagar's dedication to science is exceptional (as well as his manner of dealing with people and his appearance). I hope to follow suit. It has been great working with the energetic Dr. Hill. I admire 
his drive for science and his candor. Thanks to my other committee members; Drs Jones, Prough and Dean for their insightful comments. Thanks to the Department of Biochemistry and Molecular biology for giving me the opportunity to study in the USA. Thanks to all lab mates, to my brothers and sisters of the Fern Valley Congregation of Jehovah's Witnesses, and to my fellow Cameroonians in Louisville. I will forever be grateful for the knowledge I have amassed and I can't wait to continue putting it in use and inspiring others. Above all, greatest thanks to the most high God whose name is Jehovah. It is because of Him that everything (even science) is existing - Revelation 4:11. 


\section{ABSTRACT \\ REGULATION OF VASCULAR SMOOTH MUSCLE CELL PHENOTYPE \\ Joshua K Salabei, \\ October 30, 2012}

Vascular injury and chronic arterial diseases such as atherosclerosis and restenosis result in exposure of vascular smooth muscle cells (VSMCs) to increased concentrations of growth factors triggering a change from a contractile to a synthetic phenotype. The mechanisms by which growth factors trigger VSMC phenotype transitions remain unclear. Because phenotype switching involves the removal of contractile proteins, we hypothesized that autophagy is an essential modulator of VSMC phenotype. Also, the conversion to the synthetic phenotype is accompanied by cellular changes that trigger metabolic changes to meet the increasing demands for cellular energy. Little is known however about the contributions of different substrates metabolized for energy production and how these metabolic changes integrate with phenotype switching and VSMC proliferation. We further hypothesize that metabolic changes induced by PDGF are required for phenotype switching and proliferation. Studies presented here show 
that: 1) Treatment of VSMCs with platelet-derived growth factor PDGF-BB results in activation of autophagy which is required for degradation of contractile proteins during phenotype switching; 2) Increased glycolytic flux caused by PDGF treatment is required for expression of the synthetic cytokine osteopontin and for proliferation; and 3) Mitochondria fragmentation and increased fatty acid oxidation are required for PDGF-induced VSMC proliferation.

These results support the overall hypothesis that autophagy and changes in intermediary metabolic pathways are modulators of PDGF-induced phenotype transition. Our findings suggest that autophagy-directed therapies may provide effective treatments against vascular diseases such as atherosclerosis and restenosis. 


\section{TABLE OF CONTENTS}

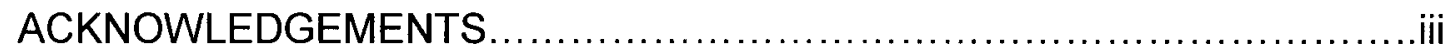
ABSTRACT. V

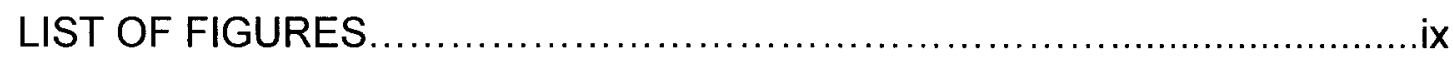

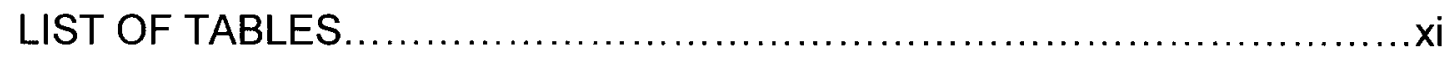

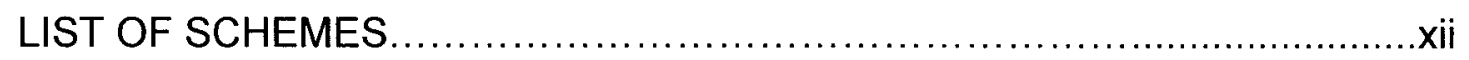

\section{CHAPTERS}

I. GENERAL INTRODUCTION........................................ ROLE OF VASCULAR SMOOTH MUSCLE CELLS IN VASCULAR

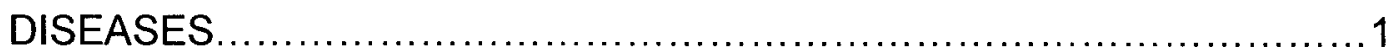

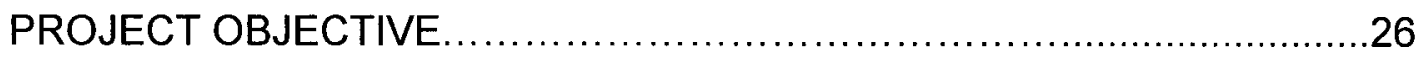

II. PDGF-MEDIATED AUTOPHAGY REGULATES VASCULAR SMOOTH MUSCLE CELL PHENOTYPE TRANSITION AND RESISTANCE TO OXIDATIVE STRESS

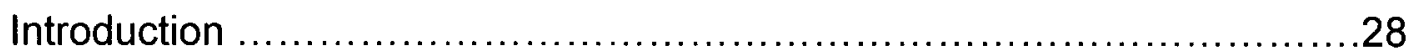

Experimental procedures.......................................... 30 
Results.

Discussion

III. REGULATION OF VASCULAR SMOOTH MUSCLE CELL PROLIFERATION AND PHENOTYPE SWITCHING BY O-GICNAC

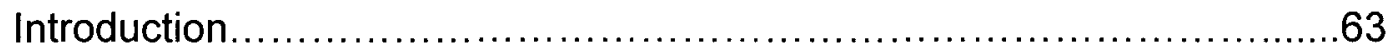

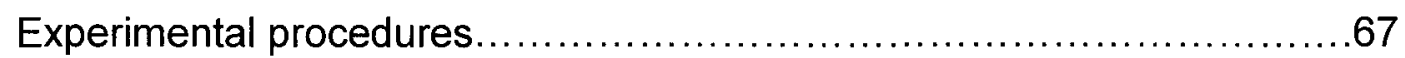

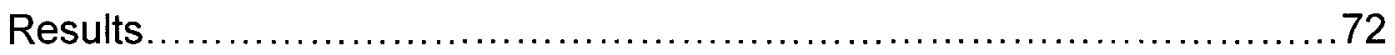

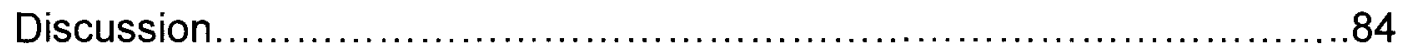

IV. MITOCHONDRIA FRAGMENTATION AND FATTY ACID OXIDATION REGULATES VSMC PROLIFERATION

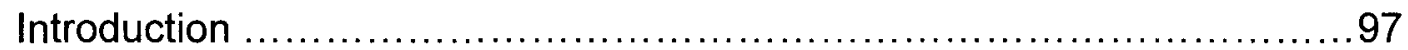

Experimental procedures.............................................100

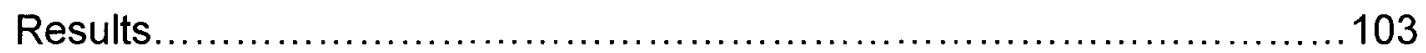

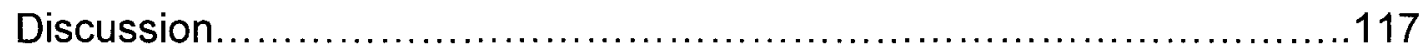

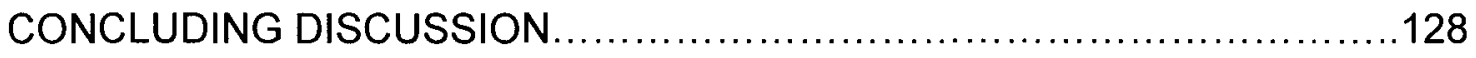

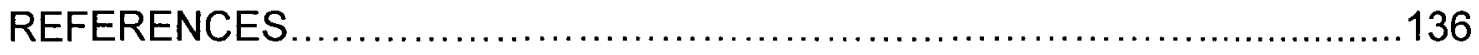

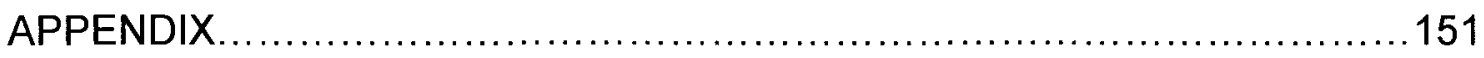

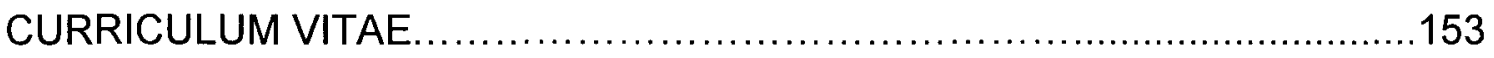




\section{LIST OF FIGURES}

FIGURE

PAGE

1. PDGF induces a contractile-to-synthetic phenotype switch in VSMCs....39

2. PDGF-BB induces autophagy in VSMCs.

3. PDGF-BB increases autophagosome formation and promotes extensive vacuolization

4. Inhibition of autophagy prevents PDGF-induced phenotype switching ....54

5. Spautin 1, an inhibitor of autophagy, prevents PDGF-induced phenotype switching 56

6. Inhibition of the proteasomal pathway does not inhibit PDGF-induced contractile protein degradation

7. Synthetic VSMCs are resistant to HNE-induced toxicity. 60

8. Spautin 1 does not interfere with PDGF-induced Akt or Erk1/2 signaling

9. PDGF-BB induces VSMC proliferation and expression of osteopontin.....74

10. PDGF-BB induces increase in glycolytic flux. .77

11. PDGF-induced glycolytic flux is required for proliferation and osteopontin expression. 
12. Inhibiting glycolytic flux increases flux to the HBP

13. Thiamet-g increases protein O-GIcNAcylation in VSMCs.

14. Increased protein O-GIcNAcylation inhibits PDGF-induced VSMC

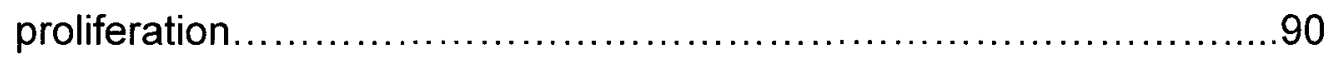

15. Thiamet-g does not inhibit PDGF signaling ..........................92

16. Increased protein O-GIcNAcylation inhibits PDGF-induced osteopontin

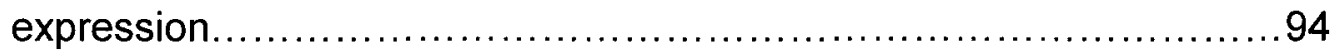

17. Thiamet-g causes delayed cell cycle progression.......................96

18. Increased fatty acid oxidation is observed in synthetic VSMCs.........107

19. Increased FAO in synthetic VSMCs is not due to increased mitochondria

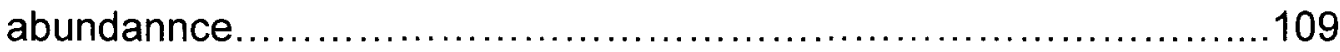

20.PDGF induces mitochondria fragmentation in VSMCs................113

21. FGF-1 does not induce mitochondria fragmentation/FAO ..............115

22. PDGF-induced mitochondria fragmentation causes increase FAO ......121

23. Inhibition of mitochondria fragmentation inhibits PDGF-induced

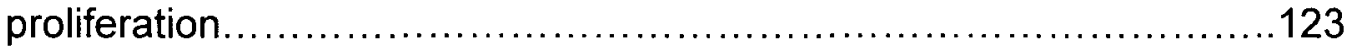

24. FAO is required for PDGF-induced proliferation..................... 125

25. Mitochondria fragmentation and FAO are not prerequisites for PDGFinduced contractile protein degradation 


\section{LIST OF TABLES}

TABLE

PAGE

1. Factors regulating VSMC phenotypes................................11 


\section{LIST OF SCHEMES}

SCHEME

PAGE

1. Cross sectional area of a human artery ..............................

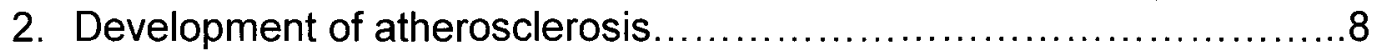

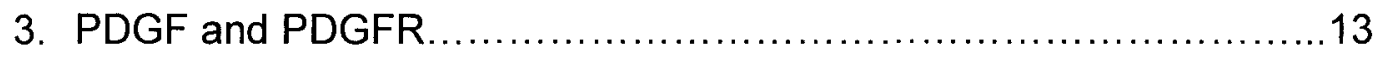

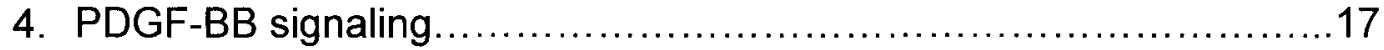

5. Measurement of oxygen consumption rates....................... 105

6. Regulation of phenotype switching ............................... 135 


\section{CHAPTER 1}

\section{GENERAL INTRODUCTION \\ ROLE OF VASCULAR SMOOTH MUSCLE CELLS IN VASCULAR DISEASES}

Despite advances in modern medicine, cardiovascular disease (CVD) remains the leading cause of mortality in the United States accounting for $35 \%$ of all deaths with about 1 in 3 American adults having one or more type of CVD [1]. According to the CDC, the total costs related to CVD in 2008 were in excess of $\$ 450$ billion, and these are expected to increase in the future [1-4]. Although CVD-related deaths have declined in high income countries, mortality have increased in low and middle income countries [5]. These differences in mortality due to CVDs are attributable to modifiable risk factors such as healthy eating, exercise, air pollution, and smoking [6].

Cardiovascular diseases encompass all diseases affecting the cardiovascular system, which include the heart and peripheral blood vessels. One of the most common CVD is atherosclerosis, i.e., the buildup of cholesterol in the artery walls of blood vessels supplying the heart (coronary heart disease) or supplying the peripheral tissues (peripheral arterial disease) [7]. The onset of atherosclerosis begins early in life from childhood whereby there is a gradual deposition of cholesterol and oxidized low density lipoprotein (LDL) in the subintima of arteries leading to the formation of an atherosclerotic plaque. Plaque formation is caused 
by macrophage-stimulated inflammatory reactions that ultimately leads to plaque rupture (or thrombosis) [8]. Thrombosis may lead to heart attack and/or stroke, which are ranked amongst the top three major causes of death in the United States [9].

Physiology of the healthy human artery. The mature healthy human artery is made up of 3 principal layers: the intima-composed of endothelial cells (ECs), the media-composed of primarily vascular smooth muscle cells (VSMCs) and the adventitia-composed of fibroblasts and connective tissues (Scheme 1). The complexity of the human artery has developed over time because the need for an efficient blood delivery system became needed as organisms became more complex. The different layers of the arteries have thus evolved to meet the needs of supplying oxygen and nutrients to distal organs and tissues. The endothelium is a thin layer of ECs allowing diffusion of important factors from blood to underlying layers and tissues. The media layer is found underneath the endothelium and it is composed of fully differentiated vascular smooth muscle cells, which express a repertoire of contractile proteins such as $\alpha$-smooth muscle actin. Thus, the principal function of the media is contraction and regulation of vessel tone and blood flow. This layer is innervated by the sympathetic and parasympathetic nervous systems and therefore the regulation of this layer is tightly controlled [10-11]. Beneath the media is the adventitia, comprised mostly of fibroblasts and connective tissues. This layer provides mechanical support and stability to the blood vessels. 
The formation of nascent blood vessels occurs via two processes; vasculogenesis, the formation of nascent blood vessels, and angiogenesis, the sprouting of new blood vessels from existing blood vessels. Expression of Vascular Endothelial Growth Factor (VEGF) is critical during vasculogenesis and angiogenesis [12]. VEGF-A stimulates the CD31+ and CD34+ VEGF receptorpositive angioblasts to initiate vessel formation and set in motion molecular events that eventually lead to mature a vascular network [13]. VEGF-A stimulates angioblasts to differentiate into endothelial cells forming nascent tubes [14]. The endothelial layer in nascent tubes and mature blood vessels is usually a single cell thick, allowing for rapid exchange of materials between blood and underlying tissues. The nascent endothelial tubes formed after VEGF stimulation are stabilized by cells of the mural compartment, particularly VSMCs. Under the influence of platelet derived growth factor (PDGF) and other growth factors such as transforming growth factor $\beta$ (TGF- $\beta$ ), VSMCs form an outer layer beneath the endothelial cells. This stabilization is followed by extracellular matrix (ECM) deposition [15]. At the level of small arteries, i.e., arterioles, smooth muscle cell organization is relatively simple with a single smooth muscle cell wrapped completely around the lumen of the nascent endothelial tube [16]. In larger arteries where there are multiple smooth muscle cell layers, VSMCs are arranged close to perpendicular with the axis of blood flow [16]. This arrangement aids lengthening of blood vessels during high pressure thus regulating vessel tone and blood distribution. Beneath the media layer of VSMCs is the adventitia layer composed of fibroblasts and other ECM components. The 
main function of the adventitia is structural because it attaches the blood vessel to the basement membrane; however, the adventitia is increasingly recognized to play non-structurally related functions because changes in this layer were amongst the earliest structural changes found in diseased vessels [17].

\section{Atherosclerosis involves the buildup of cholesterol and oxidized LDL in the}

artery wall: During atherogenesis, accumulation of cholesterol and oxLDL in the subintima space of the artery [18-19] leads to recruitment of monocytes (from the circulation) through cell junctions of the endothelial layer into the subintimal space [16-17] (Scheme 2). In the subintima, monocytes differentiate into macrophages and continuously take up excess lipids while failing to return into circulation (i.e., impaired efferocytosis). This repeated failure of the innate immune responses to clear subintimal LDL results in the deposition of lipidloaded macrophages (foam cells) which in turn induces vascular inflammation in the vessel wall [20]. Foam cell formation is also thought to occur through VSMC engulfment of subintimal lipids [21-22]. Most often, lesions are covered and stabilized by VSMCs which are thought to migrate from the media layer to form a neointima or protective cap [21-22]. However, continuous failure to clear foam cell macrophages and increased vascular inflammation beneath such caps progressively leads to weakening of the protective cap. Such advanced lesions when present in areas of high stress, for example in arterial branches, often lead to plaque rupture and thrombosis leading to heart attack or stroke. 
Scheme 1

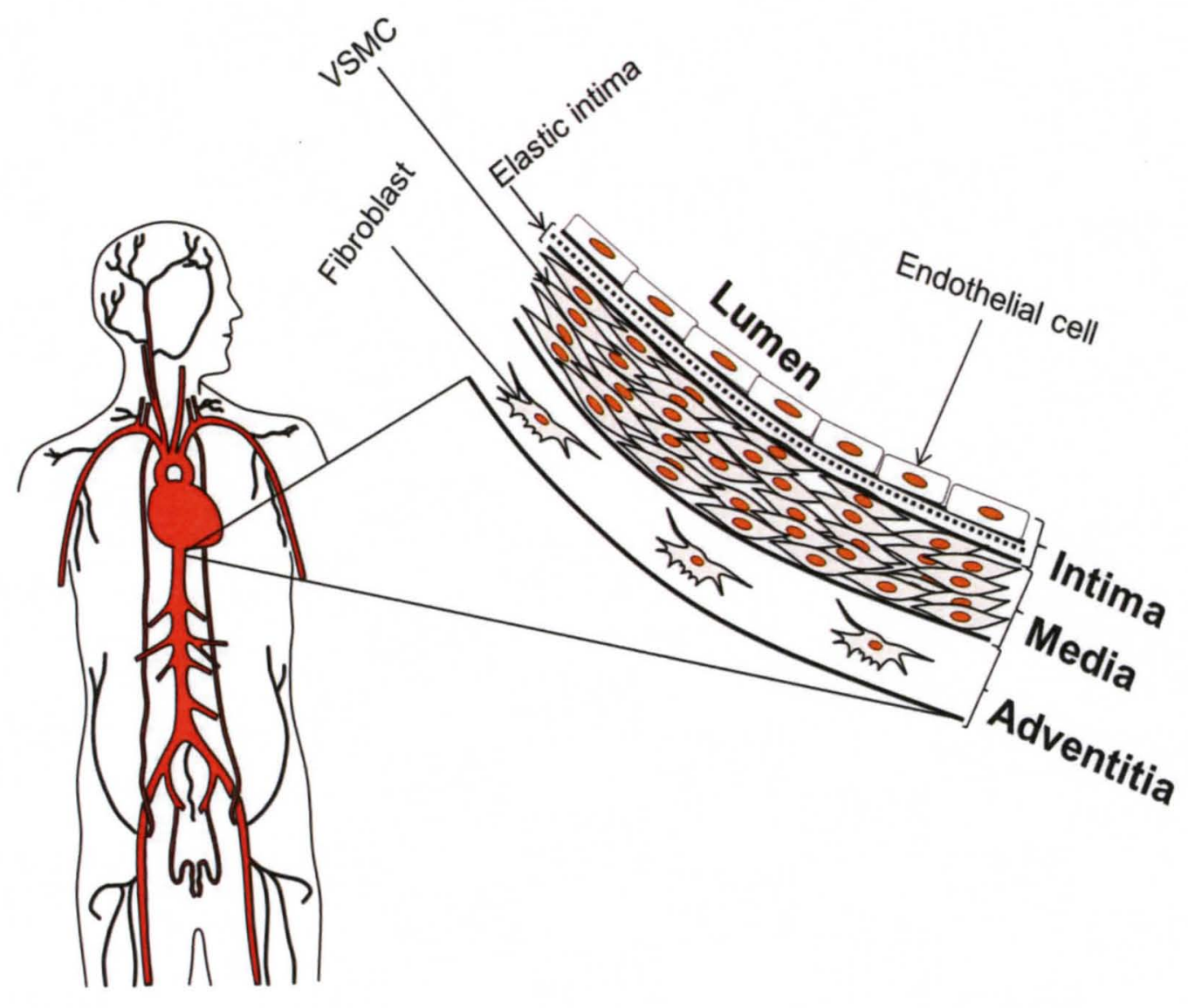

Cross sectional area of a human artery 
The majority of atherosclerotic lesions in humans go unnoticed because of the formation of the neointima that covers and stabilizes these lesions [23]. In cases where the formation of the neointima is thick enough to hinder blood flow through a vessel, slight chest pain or angina is often felt [24]. In other cases where there is severe or complete obstruction of blood flow (such as after thrombosis), angioplasty, a mechanical surgical intervention, is used to widen the obstructed artery after which a (drug eluting) stent is placed to prevent VSMC proliferation. However, re-occlusion (or restenosis) of previously opened (restored) arteries characterized by excessive proliferation of underlying VSMCs that migrate luminally and form a neointima is common [25-26]. For example, a study with a total of 1,012 patients who were randomly assigned treatment with sirolimuseluting ( $n=503$ ) or paclitaxel-eluting stents $(n=509$ ) showed that $\sim 28 \%$ of subjects needed revascularization at some later time [27]. Although the outcome of such clinical studies depends on the demographics of the population in question (such as the presence of diabetes), it highlights restenosis as an important question that continually warrants our attention. Endothelial damage caused by angioplasty or diseased conditions is thought to cause increased exposure of underlying VSMCs to growth factors such as PDGF and other cytokines resulting in their migration (from the media) and hyperproliferation in the neointimal region [28]. Currently, drug-eluting stents (DES) are often placed in arteries after the plaque has been cleared, the goal of which is to inhibit VSMC proliferation and restenosis. However, despite these interventions, restenosis continues to be problematic especially in certain classes of patients such as 
those suffering from diabetes, where the restenotic process is more severe, often leading to increased neointima formation and total vessel occlusion [29-31]. This can result in increased incidence of myocardial infarction, heart failure [32] and vasculopathy, which contribute significantly to morbidity and mortality ascribed to diabetes [33-35].

Phenotype switching in VSMCs: VSMCs in healthy arteries express a repertoire of contractile proteins, signaling molecules and ion channels required for their contractile function [36]. A number of SMC-specific contractile genes have been used to identify the contractile phenotype. These include smooth muscle isoforms of $\alpha$-actin (SM- $\alpha$-actin), SM myosin heavy chain (SM-MHC), calponin, SM22a and smoothelin [37-45]. Although these SMC marker genes are expressed in the differentiated phenotype, they are also transiently expressed in other cell types during development, disease progression and tissue repair [46]. Because of this, multiple marker genes are required for rigorous identification of fully differentiated VSMCs, and no one SMC marker gene can exclusively be used for the identification of VSMCs. 


\section{Scheme 2}

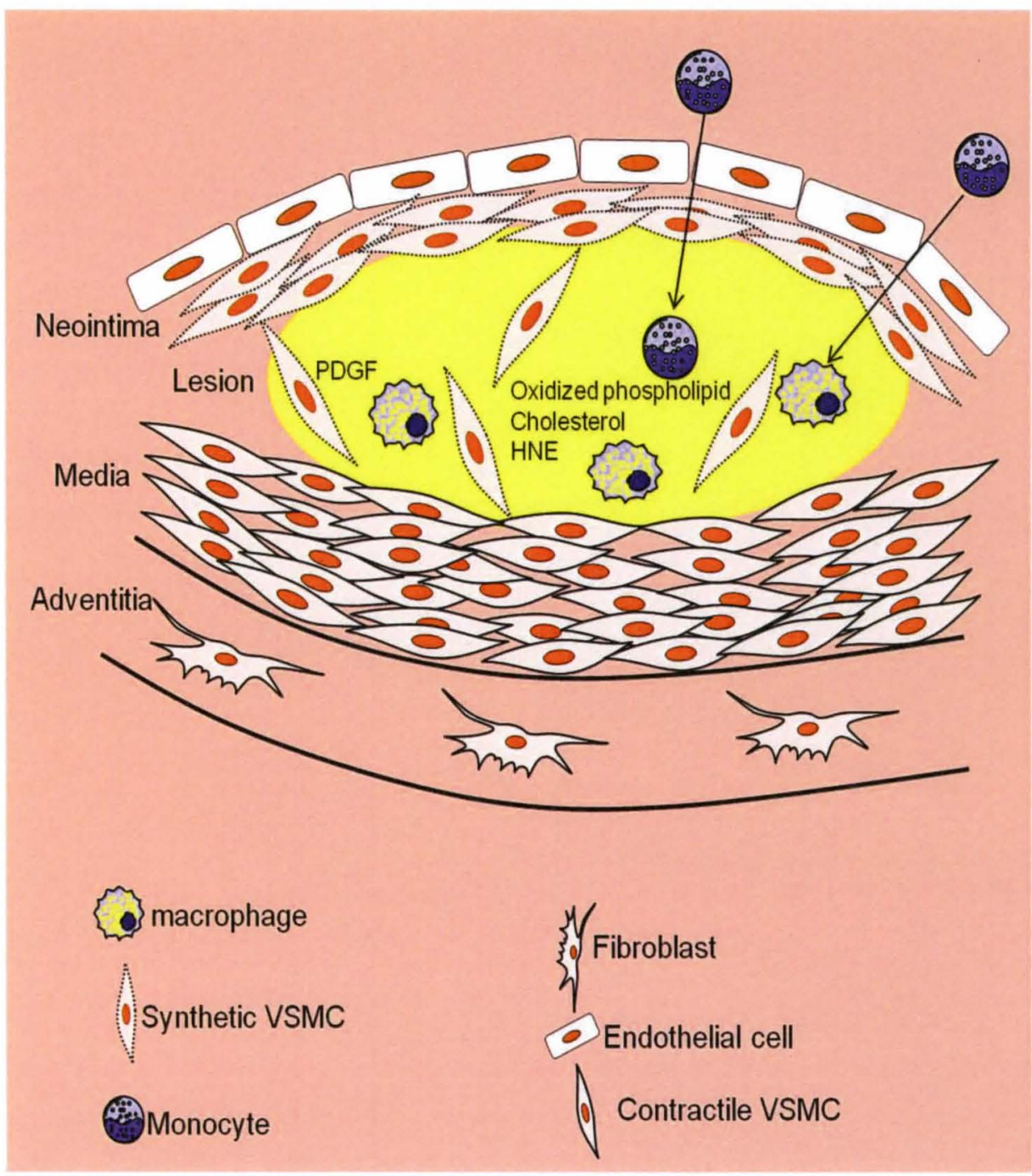

Development of atherosclerosis 
Unlike skeletal and cardiac muscle cells, which are terminally differentiated [47], VSMCs have a unique characteristic of plasticity that enables them to switch to a less contractile or synthetic phenotype (de-differentiation). The synthetic phenotype is typically characterized by loss of expression of contractile proteins and increased expression of synthetic proteins such as osteopontin [28, 48]. However, there appears to be a phenotype gradient between the contractile and synthetic phenotypes, such that, depending on environmental cues, VSMCs can develop features of both phenotypes or reverse their phenotype from a dedifferentiated to a contractile phenotype [49].

Many factors regulate the development or maintenance of the contractile and synthetic phenotypes. Some of these factors have been summarized in Table 1. Synergistic effects of signals or synergistic effects of multiple pathways on VSMC dedifferentiation have also been reported. For example, it was shown that PDGF and IL1- $\beta$ can act synergistically to promote the development of the synthetic phenotype [50]. Extracellular matrix proteins such as polymerized collagen have been shown to cause down regulation of genes such as thrombospondin and fibronectin, which are known to be upregulated in injured vessels [51]. Other in vitro studies have further confirmed that VSMCs cultured on a fibronectin coated surfaces are induced to become more synthetic, while when cultured on a laminin-rich surface, their contractile phenotype is maintained [52-53]. The effects of these different ECM proteins on VSMC phenotype is known to be mediated through integrin-cell surface receptors. Wang et al [54] showed that the ECM cartilage oligomeric matrix protein (COMP) maintained VSMCs in their 
contractile phenotype and the effects of COMP were mediated through $\alpha_{7} \beta_{1}$ intergrin, a major laminin receptor in VSMCs. The role of growth factors in regulating VSMC phenotypes has also been extensively studied. Transforming growth factor beta, insulin growth factor- 1 and angiotensin are known examples that promote the contractile phenotype (see Table 1). In contrast, basic fibroblast growth factor (bFGF) and PDGF promote the synthetic phenotype, although only PDGF has been identified as a factor that can selectively and directly promote phenotypic modulation of VSMCs [55-56].

PDGF as a mitogen and an inducer of VSMC phenotypic switching: The platelet derived growth factors (PDGFs) constitute key mitogens for cell types of mesenchymal origin including smooth muscle cells. The PDGFs comprise a family of four different polypeptides, which include PDGF-A, B, C and D. These polypeptides form homo- or hetero-dimers with five known dimeric isoforms: PDGF-AA, AB, BB, CC and DD. PDGF-A and $B$ are known to heterodimerize while PDGF-C and D only form homodimers [57] (Scheme 3). The PDGFs signal through tyrosine kinase receptors, i.e., PDGFR- $\alpha$ and PDGFR- $\beta$. Both PDGFR- $\alpha$ and $-\beta$ can form homo or heterodimers that can be activated to varying degrees by the PDGFs [57]; however, PDGF-BB has been shown to be one of the most potent forms to activate the PDGFR signaling cascade, leading to VSMC dedifferentiation and proliferation [58]. PDGF-BB, to date, is the most potent stimulator of VSMC proliferation, migration and phenotype switching [56]. 


\section{Factor}

Effects on SMC

phenotype

Growth factors

- PDGF-BB

- bFGF

- Angiotensin II

- Transforming growth factor

- Insulin and IGF-1

Injury/atherogenic stimuli

- Oxidized phospholipids

- ROS

- Cytokines

MicroRNAs

- miRs $145,143,195,25,1$

- miRs $21,221,222,126$

Basement matrices

- Monomeric collagen

- Polymeric collagen

- Fibronectin

- Matrigel

Extracellular matrix components

- ADAMST7

- Cartilage oligomeric matrix protein

- Osteopontin

Pharmocoligical inhibitors

- Rapamycin

- Lovastatin

- Spautin1
- Promotes synthetic

- Promotes synthetic

- Promotes contractile

- Promotes contractile

- Promotes contractile

- Promotes synthetic

- Promotes contractile

- Promotes synthetic

- Promotes contractile

- Promotes synthetic

- Promotescontractile

- Maintains contractile

- Promotes synthetic

- Promotes contractile

- Promotes synthetic

- Maintains contractile

- Promotes synthetic

- Promotes contractile

- Promotes contractile

- Promotes contractile
- [58-59]

- [60-61]

- [62]

- [63-64]

- [65]

- [66-67]

- [68]

- $[50,69]$

- [70-74]

- [75-76]

- [50]

- $[50]$

- [77]

- [78]

- [79]

- $[80]$

- $[81]$

- $[82-83]$

- [84]

- Unpubli shed

Table1: Factors regulating VSMC phenotypes 
PDGF signals via multiple pathways: In the VSMC, PDGF-BB signals principally through PDGFR- $\beta \beta$ and activates several biological pathways responsible for VSMC growth and dedifferentiation [85]. These pathways have also been shown to play a role in the pathogenesis of diseases such as atherosclerosis and restenosis [49]. PDGF-BB activates the Akt, Erk1/2 and p38 MAPKs, as well as the JNK pathways. Activation of the Erk1/2 and Akt pathways is known to play important roles in PDGF-induced VSMC proliferation, migration and phenotype switching [50]. The activation of the other MAPKs by PDGF also has profound effects on SMC behavior. Zhan et al [86] infected VSMCs with AdDN-p38 or Ad-DN-JNK and found that PDGF-induced SMC migration was suppressed by $81 \%$, and $77 \%$, respectively. Zhan et al also showed that Ad-DNp38 inhibited neointima formation after balloon injury of a rat carotid. This role of p38 MAPK in neointima formation was independently confirmed by Proctor et al [87]. 


\section{Scheme 3}

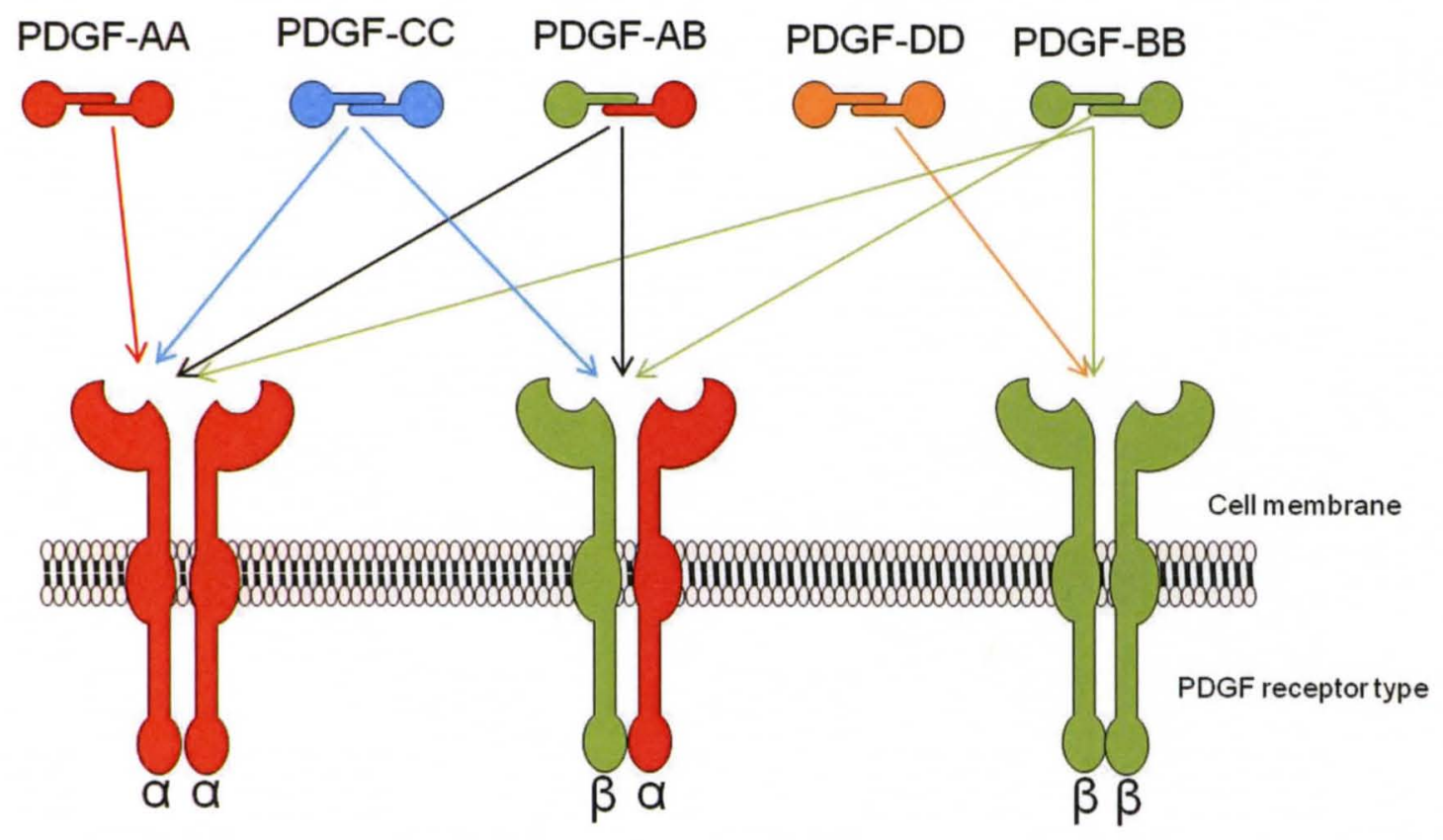

Platelet derived growth factors and platelet derived growth factor receptors 


\section{Multiple pathways and transcription factors are required for PDGF-induced}

phenotype switching and proliferation: PDGF-BB-induced phenotype switching in VSMCs occurs, in part, through transcriptional regulation of promoters upstream of SMC marker genes resulting in their down regulation. A critical transcription factor controlling expression of VSMC marker genes is serum response factor (SRF). SRF binds to $\mathrm{CC}\left(\mathrm{A} / \mathrm{T}\right.$-rich) ${ }_{6} \mathrm{GG}(\mathrm{CArG})$ boxes in the promoter regions of VSMC marker genes to control their expression [88]. CArG boxes are also present in the promoter regions of genes involved in ECM production, migration and proliferation of VSMCs [89]. This suggests that any factor that affects SRF binding to its response element is likely to affect multiple genes that regulate cell phenotype. SRF utilizes coactivators such as myocardin, which is expressed exclusively in SMCs and cardiomyocytes [90]. Generally, myocardin forms a complex with SRF on CArG boxes to promote contractile gene expression [90] (Scheme 4). In its simplest model, SRF is seen as a platform molecule that, when bound to the CArG box, recruits other molecules to form complexes. These complexes either activate or suppress expression of SMC marker genes. PDGF is known to suppress complex formation of myocardin and SRF and thus promotes the down regulation of contractile gene expression [91] (Scheme 4). Simultaneously, PDGF promotes complex formation of SRF with other transcription factors such as Elk-1, which is also known to play a role in down regulating VSMC contractile gene expression [91].

Another important transcription factor known to play a role in PDGF-induced VSMC dedifferentiation is Kruppel like factor 4 (Klf4) [92-95]. Klf4 is up-regulated 
upon PDGF stimulation, and siRNA suppression of Klf4 was shown to inhibit PDGF-induced down-regulation of contractile genes [96]. This regulation of transcription factors is one level of control regulating VSMC phenotype switching. CArG boxes in promoter regions are epigenetically marked with distinctive patterns of posttranslational modifications in the form of histone methylations and acetylations [58, 97]. These modifications regulate cis and trans DNA elements also necessary for the control of expression of smooth muscle cell marker genes [98]. In addition to regulating transcription factors, PDGF-BB is also known to alter these posttranslational modifications through decreased histone $(\mathrm{H} 4)$ acetylation [99].

\section{Phenotype switching induced by PDGF is accompanied by changes in ECM}

components: The synthetic phenotype of VSMCs is characterized by increased ECM secretion which is known to play an important role during cap formation in atherosclerosis [36]. During phenotype switching, PDGF induces changes in ECM components such as MMPs [100] and plasminogen activator inhibitor protein 1 (PAI-1) [86]. An important class of MMPs affected by PDGF and known to affect the progression of atherosclerosis and restenosis is the disintegrin and metalloproteinase with thrombospondin motifs (ADAMTS). Members of the ADAMTS family bind to and degrade other ECM components. ADAMTS-7, in particular, increases after balloon injury of the carotid artery, and its overexpression exacerbates neointima formation [101] (see Table 1). An important ECM protein degraded by ADAMTS-7 is COMP (or cartilage oligomeric matrix protein) [102]. Unlike ADAMTS-7, COMP expression decreases after 
balloon injury and its overexpression inhibits neointima formation [102]. COMP is known to interact with the integrin receptor $\alpha_{7} \beta_{1}$ thereby indicating that the intergrin receptors play important roles during PDGF-induced VSMC changes. The alteration of the ECM also serves the purpose of releasing growth factors and promoting VSMC migration during vascular remodeling under pathology.

\section{PDGF-induced osteopontin expression is important in VSMC remodeling: A} key synthetic gene in the context of vascular disease induced upon PDGF stimulation is osteopontin (OPN) [103]. OPN is also known as bone sialoprotein 1(BSP-1) or secreted phosphoprotein 1 (SPP1) and is secreted by other cell types like macrophages and endothelial cells [104]. OPN is a cytokine with autocrine effects and is thought to play important roles in VSMC proliferation during atherosclerosis [105]. Its levels are also increased during neointima formation [103, 105-106]. In fact, it has been shown that ablation of OPN in female $\mathrm{APOE}^{-1-}$ mice completely prevented the formation of atherosclerotic plaques [107]. Leali et al [108] showed that overexpressing OPN prevented reendothelization via intergrin engagement and that endothelial cells grown on an OPN-rich basement showed reduced wound healing [108]. OPN is also associated with aortic abdominal aneurysm (AAA), the weakening and bulging of blood vessels due to excessive apoptosis of VSMCs in the media. Using microarray analysis, Zheng et al [109] found that OPN was the highest expressed gene in AAA tissues collected from patients who had no atherosclerosis but underwent AAA repair operations. Zheng and colleagues found that OPN stimulated autophagy in these patients and prolonged stimulation of autophagy 


\section{Scheme 4}

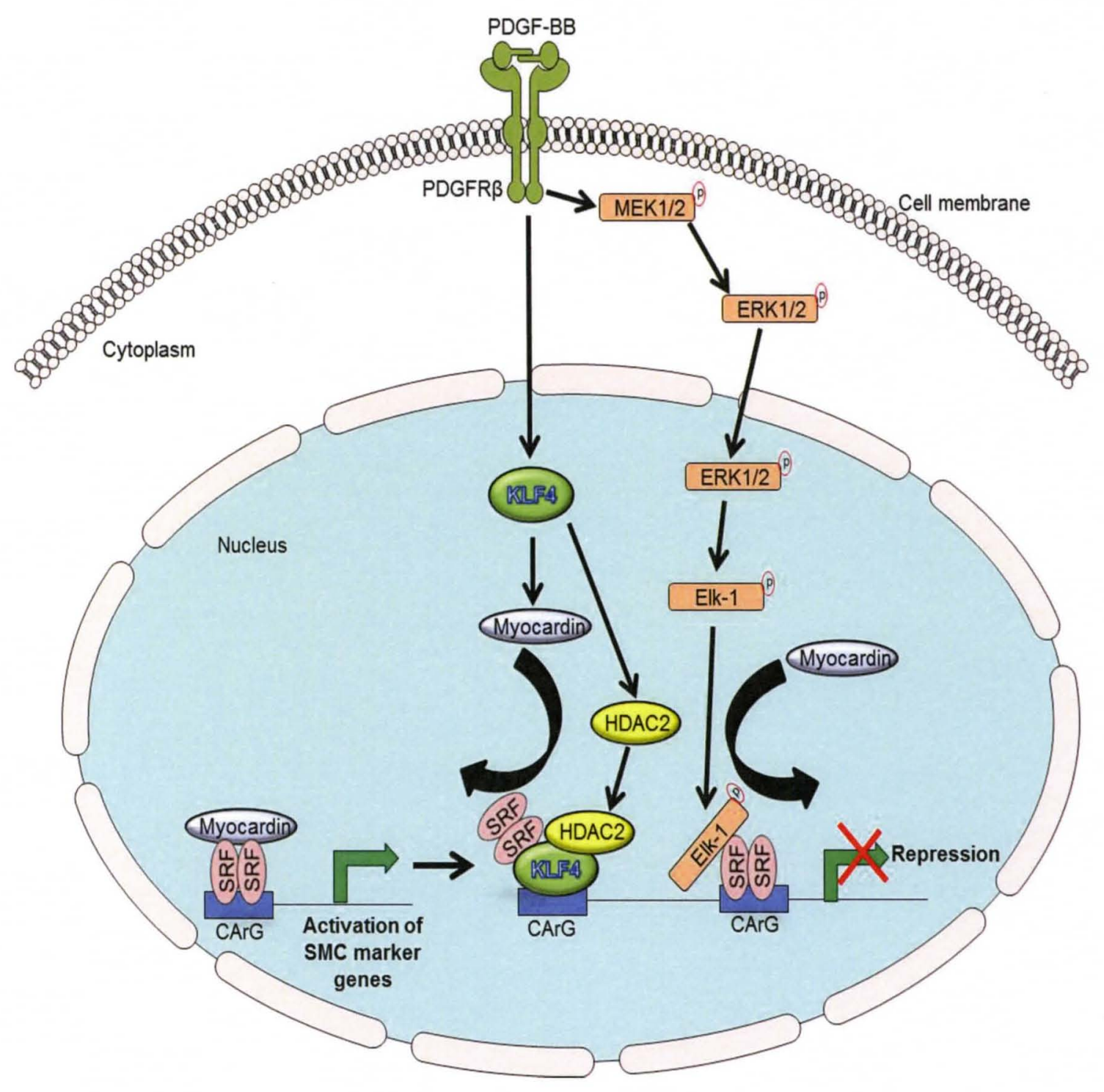

Platelet-derived growth factor-BB 
led to type II cell death of VSMCs. The autophagy induced by OPN was mediated through an integrin/CD44 and p38 MAPK-mediated pathway [110]. In patients suffering from diabetes, OPN is known to play a role in increased vascular calcification. It has been proposed that the metabolic alterations of diabetes are responsible for the increase in vascular calcification in these patients [111-112]. Because dysregulated glucose metabolism plays a key role in the development of diabetes, it was hypothesized that glucose regulates OPN expression. Based on this hypothesis and using gel mobility shift assays, Bidder et al [113] identified upstream stimulatory factor (USF) and activator protein-1 (AP1) as important regulators of OPN gene expression. The levels of USF and AP1 were in turn controlled by glucose metabolism. Mannitol and 2deoxyglucose failed to produce the same effects. A few years later, Berglund et al [114] specifically implicated the role of nuclear activator of T cell (NFAT) in hyperglycemia-dependent regulation of OPN expression in VSMCs. These studies suggest that glucose metabolism plays a key role in OPN expression and perhaps in PDGF-induced OPN gene induction. How glucose metabolism regulates OPN expression remains unclear.

Evidence of phenotype switching in atherosclerosis and restenosis: Several studies support the idea that conversion of smooth muscle cells to a synthetic phenotype plays an important role in vascular disease. For example, time dependent down regulation of $\alpha$-sm-actin was observed in autopsied tissue samples from atherectomy patients and from patients undergoing transluminal 
coronary angioplasty indicating that there was at least some change in SMC phenotype different from the contractile found in healthy blood vessels [115-116]. This finding in humans was later reproduced in experimental models of rabbit atherosclerosis. Aikawa et al [117] found that contractile genes were down regulated in the media and outermost edge of atheromas in these animals. The expression of these contractile genes showed a time dependent increase in experimental groups undergoing extensive lipid lowering treatment for 16 months after induction of atheroscelerosis. This suggests that SMCs became more contractile after lipid lowering and this corresponded to decreased atherosclerosis. A profound finding in this study is that the lipid lowering treatment caused marked reductions in ECM proteins such as MMPs and VSMCs acquired an appearance of the contractile phenotype characterized by few secretory organelles such as the golgi and endoplasmic reticulum.

Further supporting a role of phenotypic remodeling, other studies have reported that intimal SMCs undergo changes such as increased DNA synthesis, expression of proliferating markers such as PCNA and cyclins, decrease expression of contractile genes (other than a-sm-actin) and alteration of cell ultrastructure [118-120]. In 2001 it was shown that pharmacological inhibitors of PDGF receptors could inhibit VSMC proliferation, migration and neointima investment [121]. This experiment was not conclusive in providing clear evidence as to the role of PDGF in atherosclerosis because the unwanted side effects of the use of chemical inhibitors could not be rigorously ruled out. Later in 2002, using a different approach, a direct role of PDGF-induced phenotypic modulation 
began emerging clearly. Kozaki et al [122] administered PDGFR blocking antibodies to ApoE-I- mice on a Western diet and found that there was up to $67 \%$ reduction in atherosclerotic lesion size with reduced SMC investment in the neointima.

The majority of studies have thus focused on identifying signaling and transcriptional mechanisms regulating phenotype switching in diseased conditions. For example, Wamhoff et al [123] showed that mutation of a G/C repressor in the $5^{\prime}$ region of the CArG element of SM22a promoter and other SMC marker gene promoters abolished the downregulation of SMC marker genes in ApoE-/- mice on Western diet. The G/C repressor binds to the transcription factor KLF4 and interestingly PDGF-induced SMC marker gene repression is dependent on KLF4 binding to the $\mathrm{G} / \mathrm{C}$ repressor $[66,92]$. In ApoE1- animals on Western diet, KLF4 expression was markedly increased within lesions [67]. Also, KLF4 overexpression strongly inhibited the expression of myocardin, the coactivator necessary for SMC marker gene expression meanwhile conditional knockdown of KLF4 transiently delayed the repression of SMC marker genes in ApoE-/- animals on high fat diet [124-125]. These findings therefore suggest a hypothesis whereby PDGF-induced phenotype switching during atherogenesis occurs via KLF4 binding to the G/C repressor of SMC marker genes thereby causing repression (Scheme 4). Few studies have looked at postranscriptional changes such as protein degradation and how these changes could impair phenotype switching. 


\section{Protein degradation during phenotype switching and in vasculo-proliferative}

disorders: In order to maintain homeostasis, mammalian cells need to balance synthetic and degradative processes. Cellular degradation in mammalian cells is carried out by two well described mechanisms: the proteasomal and autophagic pathways [126-128].

Few studies addressing the role of the proteasomal pathway in vascular smooth muscle cell remodeling and proliferation during arterial disease have been reported. For example, the proteasomal inhibitors lactalysin and MG132 inhibited VSMC proliferation in culture and after arterial injury in an animal model of restenosis [129-130]. These inhibitors were found to stall cells at the S-phase of cell cycle probably through up-regulation of the cell cycle inhibitor p21. However, it has not been determined whether these drugs inhibited phenotype switching, which is generally thought to precede VSMC proliferation.

The role of autophagy in atherosclerosis has also been documented. For example, it was recently reported that inhibiting autophagy in macrophages by silencing ATG5 or other autophagy mediators led to both increased apoptosis and plaque instability in advanced lesions [131-132], which increases the risk of MI. Perhaps, ablation of autophagy in these macrophages led to the accumulation of damaged materials (e.g., misfolded proteins, damaged mitochondria) that triggered apoptosis. Such a finding indicates a beneficial role of autophagy in atheroclerosis. Autophagy is known to play a protective role in the VSMC in lesions. For example autophagy is known to be protective against cholesterol and HNE-induced toxicity [133-134]. Autophagy was found to be responsible for the degradation of protein- 
HNE adducts thus increasing the survival of VSMCs. However, the response to HNE-induced toxicity or HNE-protein adduct formation in the different phenotypic states has never been thoroughly studied. Since synthetic VSMCs are the abundant phenotypes present in atherosclerosis, a prediction will be that these synthetic VSMCs are more resistant to oxidative insults, thus explaining their survival in this harsh oxidative environment. Also, since there is rapid turnover during phenotype conversion, autophagy might serve the purpose of degrading cellular components, releasing metabolic intermediates needed by the metabolically demanding synthetic cell [135].

\section{PDGF-induced phenotype switching and proliferation is accompanied by} metabolic changes: Proliferating cells require, in addition to ATP, large amounts of nucleotides, amino acids and lipids. Glucose and glutamine are principal substrates used for the production of ATP as well as intermediates critical for the synthesis of important biomolecules [136-137]. Although the hydrolysis of ATP is needed for important biochemical reactions important during proliferation, additional requirements also have to be met. For example, synthesis of fatty acids requires more equivalents of carbon and NADH than of ATP [138]. Total metabolism of a glucose molecule can generate up to 36 ATPs. This number of ATP molecules far exceeds the amount needed to synthesize 1 molecule of an amino acid or a fatty acid like palmitate. For this reason, the bulk of glucose catabolism in proliferating cells cannot be solely committed for ATP production. If this were the case, the resulting rise in ATPIADP ratio would ultimately decrease the flux of glucose into the glycolytic pathway and the production of key glycolytic 
intermediates needed for the production of other biomolecules by the proliferating cells would be severely impaired.

Thus, it is clear that some of the glucose has to be diverted for the formation of macromolecular precursors such as acetyl-CoA needed for fatty acid synthesis. For example, during fatty acid synthesis, glucose is converted to acetyl-CoA which is then used to synthesize citrate in the TCA in the mitochondria. The citrate molecule is then transported back into the cytosol where the acetyl-CoA is recaptured and used to add 2 carbons to the growing fatty acid chain. This reaction occurs under conditions of high ATPIADP ratio such as found in proliferating cells. For such reasons, during proliferation, the metabolism of glucose is tightly controlled and orchestrated to support the production of key metabolic intermediates needed for biomolecular synthesis. This control is achieved through growth factor signaling which regulates the activity of glycolytic enzymes such as pyruvate kinase and modulates flux of carbon through the later steps of glycolysis [139]. For example, signaling through the PI3K pathway is linked to protein synthesis and glucose uptake and utilization. PI3K signaling through Akt can regulate expression of glucose transporter, and has been shown to have direct effects on enzymes such as hexokinase and phosphofructokinase activity [140-142]. Small molecule inhibitors that inhibit PI3K signaling also lead to inhibition of glucose uptake and utilization and proliferation [143]. The process of inhibition growth factor signaling has been the principal mechanism employed to interrupt aberrant proliferation of cells under disease states. 
In the VSMCs, PDGF signals through the PI3K pathway to increase flux in glycolysis and induce proliferation [144]. This increase in glycolytic flux caused by PDGF depends on enhanced glucose uptake because when glucose in the media was replaced with 2-deoxyglucose, glycolysis and proliferation were inhibited [145]. These and several studies have thus focused on the inhibition of PDGF signaling and glucose utilization in order to inhibit VSMC proliferation.

PDGF-induced glycolysis has been shown to provide substrates to the mitochondria, increasing basal oxygen consumption while also enhancing mitochondria reserve capacity [145]. The induction of proliferation by PDGF was found not only to be dependent on increased glycolytic flux, but also on increased LDH activity because LDH expression was increased and because Llactate could support PDGF-induced proliferation in the absence of glucose [145]. These findings thus suggest that integrated coordination of glycolysis and mitochondrial metabolism is required for PDGF induced proliferation, although very few studies have interrogated the role of the mitochondria after PDGF stimulation with respect to proliferation. Also, since phenotype switching induced by PDGF is an energy expending process, it is of interest to determine the role glucose metabolism plays during phenotype switching. How mitochondrial metabolism and dynamics integrates with phenotype switching and proliferation after PDGF stimulation warrants our attention.

Thus, the work presented was undertaken to: 1) Determine the role of autophagy in phenotype switching induced by PDGF; 2) Determine the role of glucose metabolism in PDGF-induced phenotype switching and proliferation; and 3) To 
test whether changes in mitochondria dynamics regulates VSMC phenotype switching and proliferation. 


\section{PROJECT OBJECTIVE}

The goal of my research is to better understand the mechanisms that regulate the VSMC phenotype in the context of vascular disease. I hypothesize that controlled and synchronized changes in metabolism influence or coordinate cellular programs to elicit changes in SMC structure and function. Specifically, I posit that changes in cell function, such as those occurring during the contractileto-synthetic phenotype switch, require activation of protein degradation programs, such as autophagy, to remove contractile proteins and to re-structure the cell to perform specialized secretory functions. Specific aims to address the aforementioned hypothesis were 1) To examine protein degradation pathways involved during phenotype switching; 2) To determine the role of glucose metabolism with respect to phenotype switching and 3) To examine the role of mitochondria remodeling and substrate usage during phenotypic switching. A significant portion of my research was directed toward understanding the role of protein degradation mechanisms involved in growth factor-induced phenotype conversion (Chapter II). These studies have elucidated an exciting new role of autophagy in the regulation of the VSMC phenotype by showing that PDGF activates a unique type of autophagy that regulates the abundance of contractile proteins. 
Our studies have also addressed the role of metabolism in VSMC hyperproliferative responses, such as occurs in injured vessels, where alterations in pathways of intermediary metabolism may impact the proliferation of VSMCs upon stimulation with growth factors. As described in Chapter III, my studies identify a key role for glucose metabolism pathways in the regulation of the synthetic protein osteopontin. Furthermore, in Chapter IV, we found that conversion to the synthetic phenotype is associated with mitochondrial fragmentation and a switch to fatty acid oxidation, which appear to play critical roles in inducing the proliferative phenotype. Collectively, these cooperative changes in metabolism integrate to promote the development of the synthetic VSMC phenotype. These findings provide novel and fundamental insights that could be important for developing therapeutic approaches to treat or prevent vascular disease. 


\section{CHAPTER II}

\section{PDGF-MEDIATED AUTOPHAGY REGULATES VASCULAR SMOOTH MUSCLE CELL PHENOTYPE TRANSITION AND RESISTANCE TO OXIDATIVE STRESS INDUCED BY HNE}

\section{Introduction}

Vascular smooth muscle cells (VSMCs) are essential regulators of vascular function. In healthy arteries, VSMCs are located in the medial vascular layer, where they express a repertoire of contractile proteins that help to regulate vessel tone and blood flow [36]. During atherogenesis and arterial restenosis, VSMCs change from a contractile phenotype to a synthetic phenotype. This promotes their migration to the intima, increases their proliferative capacity, and promotes the synthesis of extracellular matrix proteins [146]. These changes in VSMC phenotype appear to be fundamental in regulating the composition and stability of vascular lesions [147].

While several factors have been suggested to mediate VSMC phenotype transitions, platelet-derived growth factor (PDGF) is among the most robust phenotype-modulating agents and has been shown 
to be a primary regulator of smooth muscle cell growth and proliferation. It has been shown that antibodies against PDGF [148-150] or PDGF receptors [151-152], antisense oligonucleotides to PDGF receptors [153-154], or PDGF aptamers [155] inhibit smooth muscle accumulation in the intima after balloon injury, and VSMCs lacking PDGFR- $\beta$ show strikingly diminished neointimal accumulation after carotid artery ligation [156]. Moreover, pharmacological inhibition of PDGF signaling reduces VSMC proliferation, migration, and occupancy in the neointima [157-159]. Hence, understanding how PDGF regulates VSMC phenotype may be essential for developing better and more targeted strategies for preventing or ameliorating vascular disease.

The switch from the contractile to the synthetic VSMC phenotype induced by PDGF is initiated upon PDGF binding to surface receptors, which activate several intracellular signaling pathways that ultimately regulate gene expression and cellular function. These signaling pathways have been shown to result in decreased abundance of contractile proteins and increased expression of synthetic proteins such as osteopontin $[36,106]$ and vimentin [160-161]. Such structural changes have been shown to be prerequisites for enhanced cellular proliferation of VSMCs in culture [162]. Although multiple studies have focused on identifying the molecular mechanisms involved in phenotype switching $[36,146]$, it remains unclear how VSMCs can rapidly change from the contractile to the synthetic phenotype. Previous work from our laboratory has shown that autophagy is activated in VSMCs to remove oxidatively damaged proteins [133], which can form 
large aggregates that are particularly difficult to digest. This suggested to us that the contractile apparatus, which must be removed during cellular transition to the synthetic phenotype, may also be degraded by autophagy and that autophagy might be an essential regulator of VSMC viability in diseased vessels. Therefore, we examined whether PDGF-induced transition from the contractile to the synthetic phenotype is accompanied by an increase in autophagy and whether stimulation of autophagy is required for phenotype switching. Our results support the notion that autophagy is essential for the conversion of VSMC from a contractile to a synthetic phenotype and that this increase in autophagy in synthetic cells could also function to prevent cell death due to oxidative stress.

\section{Experimental Procedures}

Materials: Antibodies against $\alpha$-smooth muscle cell-actin (a-SMA), calponin and $\alpha-$ tubulin were purchased from Sigma-Aldrich (St. Louis, MO, USA). Osteopontin antibody was purchased from Santa Cruz biotechnology (Santa Cruz, CA, USA). Recombinant FGF-1 was obtained from eBiosciences (San Diego, CA, USA). Antibodies against GAPDH, p-Akt/Akt, p-p70S6K/p70S6K, p-AMPK/AMPK and pmTOR/mTOR and LC3 were purchased from Cell Signaling (Danvers, MA, USA). Recombinant rat PDGF was obtained from R\&D Biosystems (Minneapolis, MN, USA). HRP conjugated rabbit and mouse IgG secondary antibodies were obtained from Cell Signaling Technologies (Danvers, MA, USA). The 3-methyladenine (3MA), epoxomicin, and bafilomycin A1 were obtained from Sigma-Aldrich. Goat anti- 
mouse IgG-Alexafluor 555 was obtained from Invitrogen (Grand Island, NY, USA). Spautin-1 was obtained from Cellagen Technology (San Diego, CA, USA). Ad-GFP and Ad-GFP-LC3 were obtained from Vector Biolabs (Philadelphia, PA, USA). All primers used for real time PCR were designed using the primer express software from Applied Biosystems (Carlsbad, CA, USA) and then ordered from Integrated DNA Technologies (Coralville, IA, USA). DAPI stain was obtained from Invitrogen. Electrophoresis supplies were purchased from Bio-Rad (Hercules, CA, USA). $E C L^{\circledR}$ reagents were purchased from GE Healthcare (Pittsburgh, PA, USA).

Vascular smooth muscle cell isolation: Up to 3 extracted aortas from 6-weekold male Sprague-Dawley rats were cleaned to remove all perivascular fat. Sterile capel blades were used to minced aortas in a culture dish containing DMEM and $1 \% \mathrm{P} / \mathrm{S}$. The minced aortas were then transferred into sterile $50 \mathrm{ml}$ tubes. Enzyme solution $(0.1 \%$ collagenase type $1 \mathrm{~A}, 0.05 \%$ elastase type $\mathrm{III}, 2 \mathrm{mg} / \mathrm{ml} \mathrm{BSA}$, and $2 \mathrm{mM}$ calcium chloride) made in DMEM containing $1 \% \mathrm{P} / \mathrm{S}$ was then added to the minced aortas and incubated for $1 \mathrm{~h}\left(\right.$ at $37^{\circ} \mathrm{C}$ containing $5 \% \mathrm{CO}_{2}$ ). The enzyme solution was mixed regularly during incubation. After digestion, supernatant was carefully removed and discarded. Chunks of tissues at the bottom of the tube were transferred into a new sterile tube and $5 \mathrm{ml}$ of fresh enzyme solution added and digestion carried out for about $3 \mathrm{~h}$ while monitoring degradation of tissue. Growth media $(5 \mathrm{ml})$ was added immediately after all tissue was degraded and the entire content centrifuged for $5 \mathrm{~min}$ at $1100 \mathrm{rpm}$ at $4^{\circ} \mathrm{C}$ to pellet cells. After centrifugation, supernatant was discarded followed by addition of more growth medium and 
another round of centrifugation. After the $2^{\text {nd }}$ centrifugation, cells were resuspended in growth media, counted and plated at a density of $0.4-0.6 \times 10^{6}$ cells per $25 \mathrm{~cm}^{2}$ flask containing $2.5 \mathrm{ml}$ DMEM $+10 \% \mathrm{FBS}+1 \% \mathrm{P} / \mathrm{S}$. Cells were split when $75 \%$ confluency was achieved.

Cell culture: Isolated VSMCs were grown in DMEM (Dulbecco's modified Eagle's medium; Life Technologies-Invitrogen) supplemented with $10 \%(\mathrm{v} / \mathrm{v})$ FBS (fetal bovine serum; Atlanta Biologicals, Atlanta, GA, USA) and $0.1 \%$ streptomycin/penicillin. Only cells that stained positive for calponin and $\alpha$-SMA were used in experiments. To ensure maintenance of the contractile phenotype, only cells between passages $2-7$ were used. Cells were maintained in a humidified atmosphere of air and $5 \% \mathrm{CO}_{2}$ at $37^{\circ} \mathrm{C}$. At $\sim 70 \%$ confluency, VSMCs were serumstarved in DMEM containing $0.1 \%$ FBS for $24 \mathrm{~h}$. After desired treatments, cells were rinsed twice with phosphate-buffered saline and then lysed in a protein lysis buffer containing $25 \mathrm{mM}$ HEPES, $1 \mathrm{mM}$ EDTA, $1 \mathrm{mM}$ EGTA, $0.1 \%$ SDS, $1 \%$ NP40, and $1 \mathrm{X}$ protease and phosphatase inhibitors. The Lowry DC assay (Biorad, City, St., USA) was used for measuring protein concentration of crude cell extracts.

Messenger RNA isolation and real-time PCR: Messenger RNA was isolated from RASMCs using TRIZOL reagent (Invitrogen) and the concentration was determined by measuring absorbance at $260 \mathrm{~nm}$ using a Nanodrop spectrophotometer (Thermo scientific). A $20 \mu \mathrm{l}$ reverse transcription reaction mixture containing $1 \mu \mathrm{g}$ mRNA, 10 units AMV reverse transcriptase, $0.4 \mu \mathrm{M}$ poly $\mathrm{T}$ 
primer (dT18), $0.2 \mathrm{mM}$ dNTPs, and 20 units RNasin (Promega) was subjected to complimentary DNA (cDNA) synthesis in a thermal cycler (BioRad). Two microliters of cDNA was then used for amplification of the gene of interest by real time PCR using SYBR green (WWR, Radnor, PA, USA).

Western blotting: Approximately $0.5-25 \mu \mathrm{g}$ of crude cell protein was applied to each lane of a $10.5-14 \%$ Bis-Tris- $\mathrm{HCl}$ gel and electroblotted onto a PVDF membrane. The membrane was then incubated overnight at $4^{\circ} \mathrm{C}$ using appropriate dilutions of primary antibodies. PVDF membranes were then incubated at room temperature with horseradish peroxidase-conjugated secondary antibodies. Immunoreactive bands were detected using a typhoon scanner (SA Biosciences, Valencia, CA, USA) after exposure to ECL detection reagent. Bands intensity was quantified by using the TotalLab TL120 software.

Measurement of protein-bound HNE: VSMCs were serum-starved for $24 \mathrm{~h}$ and then treated without or with PDGF $(20 \mathrm{ng} / \mathrm{ml})$ for $48 \mathrm{~h}$. After PDGF treatment, cells were exposed to $50 \mu \mathrm{M}$ HNE in HBSS for $30 \mathrm{~min}$ and then replaced with $10 \%$ FBS/DMEM. Cells were then harvested or left in the incubator for a further $3.5 \mathrm{~h}$. Cells were lysed using lysis buffer and $25 \mu \mathrm{g}$ used for Western blot to quantify protein-HNE adducts using an anti-protein HNE antibody [133].

LDH activity assay: VSMCs were serum starved for $24 \mathrm{~h}$ and then treated without or with PDGF (20 ng/ml) for $48 \mathrm{~h}$. After PDGF treatment, cells were exposed to 50 
$\mu \mathrm{M} \mathrm{HNE}$ in HBSS for 30 min and then replaced with $10 \%$ FBS/DMEM media. After $16 \mathrm{~h}$, the medium was collected and lysates were prepared by scraping cells in 100 $\mu \mathrm{l}$ of PBS containing $0.1 \%$ Triton $\mathrm{X}-100$. LDH activity was measured spectrophotometrically for $4 \mathrm{~min}$ at $37^{\circ} \mathrm{C}$ by monitoring decrease in $\mathrm{NADH}$ absorbance at $340 \mathrm{~nm}$. Cytotoxicity was then estimated by dividing the change in absorbance of the medium by the values for combined LDH activity in the medium and the lysate as follows; cytotoxicity $=\left[\left(\mathrm{Abs}_{340}\right.\right.$ media * dilution factor $) /\left(\mathrm{Abs}_{340}\right.$ Media * dilution factor $)+($ Abs lysate * dilution factor $)] * 100$. The values were then normalized relative to the control, which represented $100 \%$ cell viability [163].

Adeno-viral gene transfection: VSMCs were serum starved for $24 \mathrm{~h}$ and then infected with replication-deficient adenoviruses carrying either GFP (AdGFP) or GFP fused LC3 (AdGFP-LC3). Adenoviruses were used at a multiplicity of infection (MOI) of 100 and GFP expression was used to ascertain transfection efficiency. AdGFP or AdGFP-LC3 transfected cells were then stimulated with PDGF at the indicated times. Before imaging, cells were incubated with Hoechst stain for nuclear visualization.

Immunofluorescence confocal imaging: VSMCs were washed with PBS and then fixed in ice-cold $\left(-20^{\circ} \mathrm{C}\right)$ methanol for $5 \mathrm{~min}$. The cells were then incubated for $30 \mathrm{~min}$ at room temperature (RT) in a blocking solution (5\% BSA in PBS) followed by incubation for $1 \mathrm{~h}$ with a 1:500 dilution of anti-a-sm-actin primary antibody at room temperature. A 1:1000-diluted solution of goat anti-mouse IgG 
Alexa555 secondary antibody was used for incubation after treatment with the primary antibody. After three washes with PBS, the cells were counterstained with DAPI for 10 min at RT. The cells were then mounted on glass slides using the mounting medium and cover slips were added. a-SMA staining was visualized using a NIKON A1 confocal microscope. Cells plated on glass-bottom dishes were transfected with adenoviruses as described above. After loading with each dye, the cells were placed in phenol red-free DMEM supplemented with $25 \mathrm{mmol} / \mathrm{L}$ HEPES (pH 7.4). The cells were illuminated (488- and 568-nm lines of a krypton/argon laser), and images were acquired.

Transmission electron microscopy: VSMCs were grown on cover slips (Ladd Research, Williston, VT, U.S.A.), serum-starved for $24 \mathrm{~h}$, and then treated with PDGF $(20 \mathrm{ng} / \mathrm{ml})$ for $48 \mathrm{~h}$. Control cells were left untreated. After treatment, cells were washed with PBS and fixed with $3 \%$ glutaraldehyde in $0.1 \mathrm{M}$ sodium cacodylate buffer $(\mathrm{pH} 7.4)$ for $2 \mathrm{~h}$ at room temperature $\left(25^{\circ} \mathrm{C}\right)$. The cells were then post-fixed with $1 \%$ osmium tetroxide, sectioned and embedded in LX112 plastic. Ultrathin sections were stained with uranyl acetate and lead citrate, and electron micrographs were taken using a PhilipsCM10 transmission electron microscope operating at $60 \mathrm{kV}(\times 1000-72000$ magnification $)$ [133].

Statistical analysis: Data are mean \pm SEM. Multiple groups were compared using one-way ANOVA, followed by Bonferroni post-tests. Unpaired Student's $t$ test was used for direct comparisons. A $p$ value $<0.05$ was considered significant. 


\section{Results}

Platelet derived growth factor induces phenotype switching in VSMCs: To examine the mechanisms regulating the phenotypic transition of VSMCs, we first measured changes in molecular markers of contractile and synthetic VSMC phenotypes. For this, VSMCs were deprived of serum for $24 \mathrm{~h}$ to induce cell cycle arrest. The cells were then incubated with vehicle or PDGF-BB $(20 \mathrm{ng} / \mathrm{ml})$ for 24 and $48 \mathrm{~h}$. Relative mRNA and protein abundance was measured by RT-PCR and Western blotting, respectively. As shown in Fig. 1A, PDGF treatment for $24 \mathrm{~h}$ resulted in $a \sim 50 \%$ decreases in the mRNA levels of $\alpha$-sm-actin and calponin and a robust upregulation of osteopontin (12-fold) and vimentin (2-fold). After $48 \mathrm{~h}$, the levels of $\alpha$-SMA protein were decreased by $25 \%$, calponin abundance was decreased by $75 \%$, and osteopontin protein was elevated 2 -fold (Figure 1B and 1C). Immunofluorescence imaging of a-SMA filaments showed marked disorganization in PDGF-treated cells compared with control cells $48 \mathrm{~h}$ after PDGF treatment (Figure 1D). These results confirm previous findings $[36,50,162]$ showing that PDGF promotes phenotypic transition of VSMCs.

PDGF induces autophagy in VSMCs: The relatively rapid loss of contractile proteins suggested to us that proteolytic mechanisms may function to remove the contractile apparatus to aid in the transition from the contractile to the synthetic phenotype. Indeed, previous studies have shown that proteasome and calpain 
inhibitors affect VSMC phenotype [164-166]; however, these studies showed that proteasome inhibition counter intuitively decreases markers of the contractile phenotype [165] resulting in reduced cell contractility[166]. Therefore, we hypothesized that autophagy may be important for the removal of contractile proteins and protein complexes. To test this hypothesis, we first assessed the effects of PDGF on autophagy in VSMCs by examining LC3-II formation-an indicator of autophagy [167-168]. As shown in Fig. 2A-C, PDGF stimulation caused a time-dependent increase in LC3-II formation. A significant increase in LC3-II was observed $12 \mathrm{~h}$ after PDGF stimulation and maximal increases in LC3-II were obtained $48 \mathrm{~h}$ after PDGF treatment, suggesting that stimulation with PDGF increases autophagy. 
Figure 1. PDGF induces a contractile-to-synthetic phenotype switch in VSMCs. Indices of vascular smooth muscle cell phenotype: A) Quantitative real-time PCR following PDGF-BB $(20 \mathrm{ng} / \mathrm{ml})$ stimulation for $24 \mathrm{~h}$. Calponin, a-sm-actin and MHC II are markers of the contractile phenotype, osteopontin and vimentin are associated with a synthetic phenotype. ${ }^{*} p<0.05$ vs control, $n=3$ per group. B) Western analysis of contractile and synthetic proteins after PDGF stimulation: VSMCs were serum-starved for $24 \mathrm{~h}$ and then stimulated with PDGF for $48 \mathrm{~h}$ and $0.5-2 \mu \mathrm{g}$ protein was used for immunoblot analysis. C) Quantification of immunoblots from panel $B\left(n=3\right.$ per group, ${ }^{*} p<0.05$ vs control). D) Confocal images of $\alpha$-sm-actin (red) distribution in VSMCs treated without and with PDGF $(20 \mathrm{ng} / \mathrm{ml})$ for $48 \mathrm{~h}$. Blue staining indicates nuclear staining with DAPI (Scale bar $=20 \mu \mathrm{m}$ ). 
Figure 1
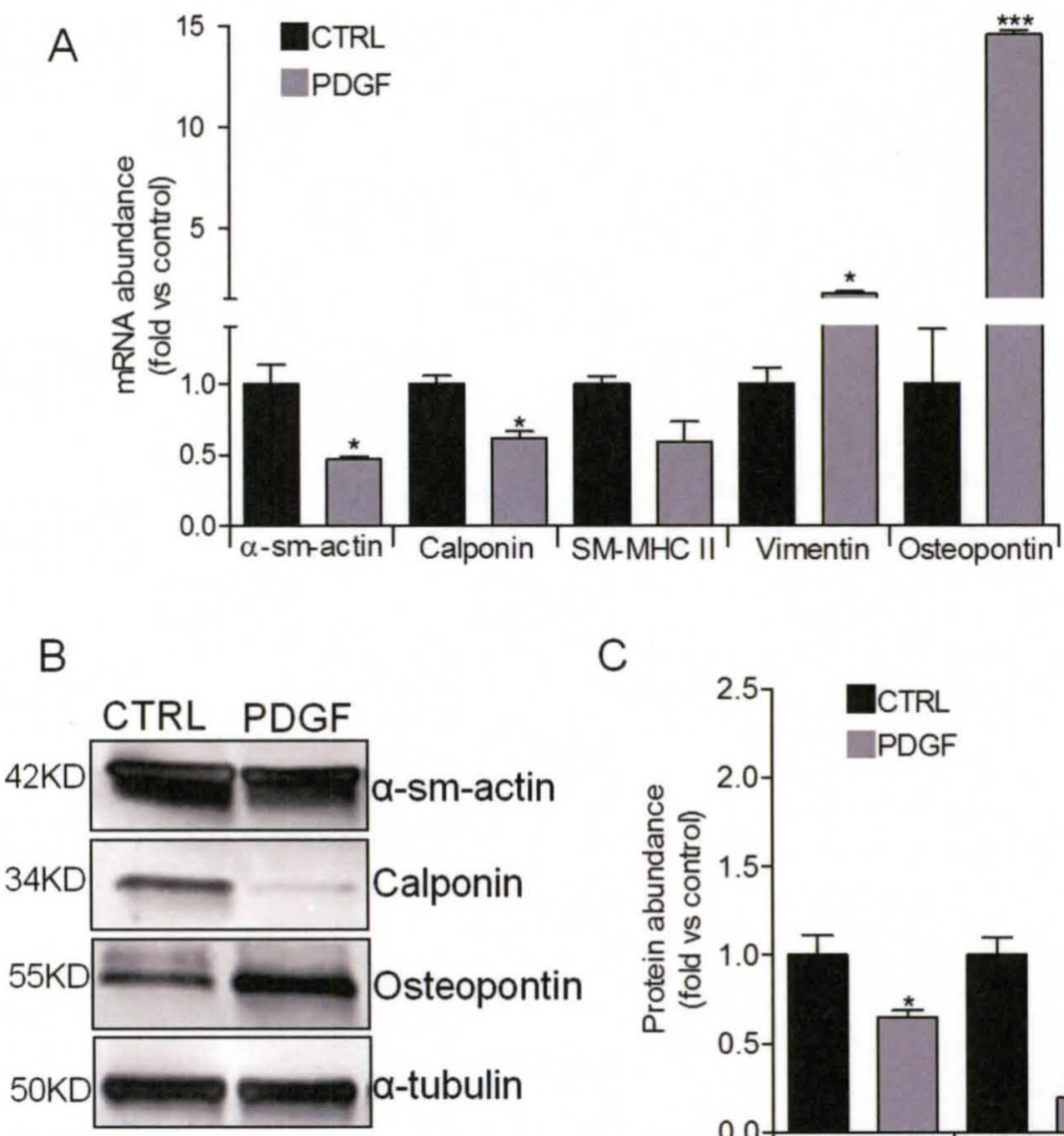

C
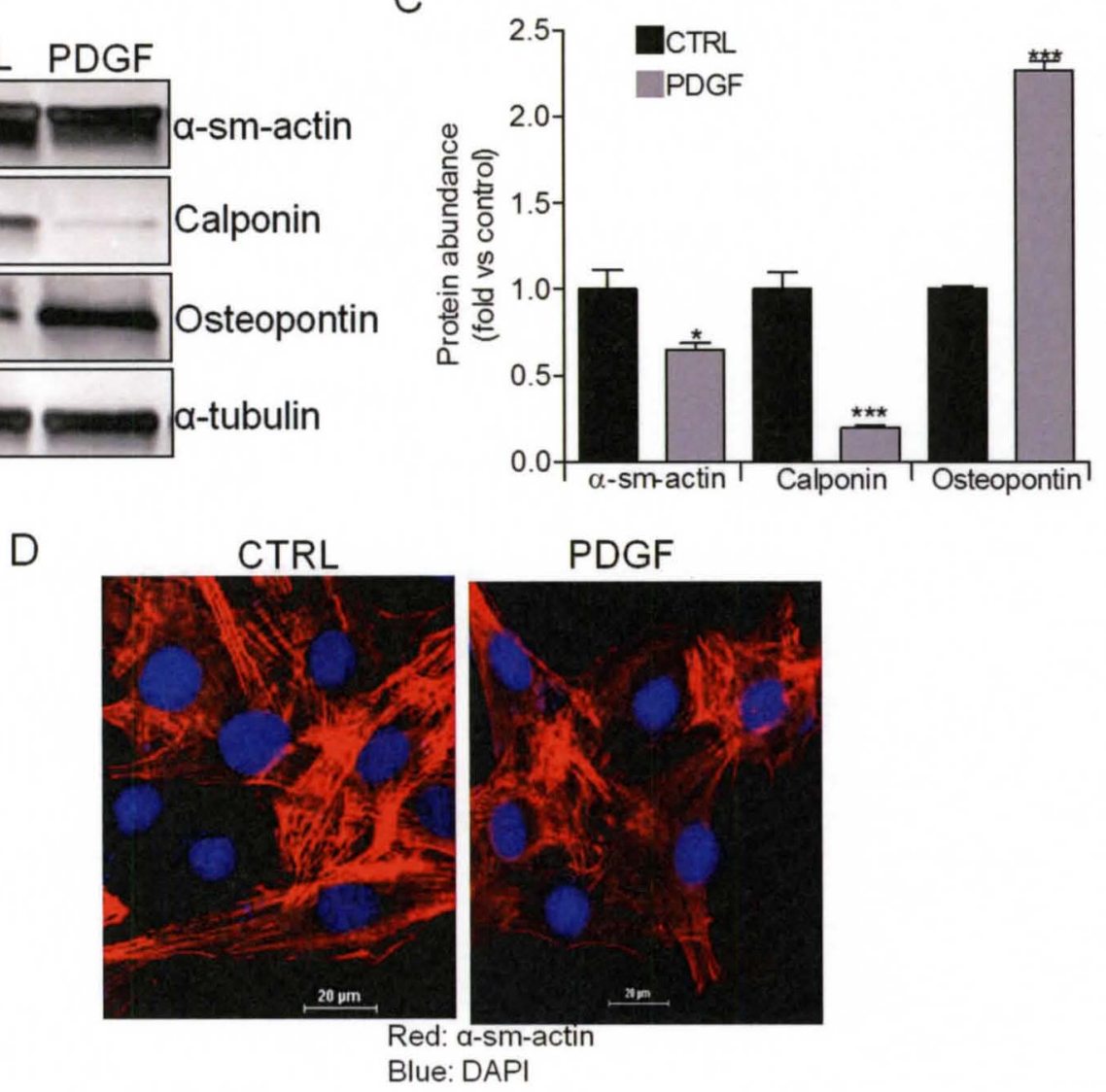
Because the formation of LC3-II is transient and the protein is rapidly degraded in the lysosome, an increase in LC3-II could also paradoxically indicate a decrease in autophagy. Hence, to determine total LC3-II formation, we inhibited its lysosomal degradation by treating cells with bafilomycin $\mathrm{A} 1$, which inhibits vacuolar-type $\mathrm{H}+-$ ATPase [168-169]. As shown in Fig. 2D-F, treatment of PDGF-stimulated cells with bafilomycin led to a greater increase in LC3-II abundance than was observed in untreated cells, indicating a net increase in LC3-II formation and upregulation of autophagic machinery in PDGF-treated cells (Fig. 2D-F). These effects appear to be specific to PDGF because treatment with fibroblast growth factor 1 (FGF-1), a known VSMC mitogen [170], did not affect the abundance of contractile proteins or increase LC3-II formation, despite increasing cell proliferation to levels similar to that of PDGF (Fig. 2G-J). To obtain further evidence for the induction of autophagy by PDGF, we transduced VSMCs with a GFP-LC3 adenovirus and assessed the formation of punctate LC3, which is indicative of its autophagosomal localization [168]. As shown in Fig. 3A-B, there was a 2-fold increase in fluorescent puncta in PDGF-treated cells, and this was further increased by bafilomycin $\mathrm{A} 1$ treatment.

PDGF induces ultrastructural changes and extensive vacuolization in VSMCs: Although LC3-II formation is a validated marker of autophagy, changes in the ultrastructure showing the formation of autophagosomes is considered the "gold-standard" for documenting autophagy. Hence, to examine ultrastructural changes, VSMCs were treated with PDGF for $48 \mathrm{~h}$ and then visualized by 
transmission electron microscopy. Transmission electron micrographs of these cells showed that treatment with PDGF $(20 \mathrm{ng} / \mathrm{ml})$ for $48 \mathrm{~h}$ caused a $\sim 3$ fold increase in autophagosomes (indicated with red arrows, Fig. 3D, E) when compared with untreated cells (Fig. 3C). In addition, PDGF treatment resulted in extensive vacuolization and consistent formation of a singular, large phagosome per cell, which may serve as a designated compartment for the digestion of intracellular materials. Collectively, these structural changes are in concordance with our immunological and fluorescence-imaging data and suggest that PDGF is a robust inducer of the autophagic program. 
Figure 2. PDGF-BB induces autophagy in VSMCs. Quantification of LC3 abundance and localization to puncta: A) Immunoblot analysis of LC3. After $24 \mathrm{~h}$ of serum deprivation, VSMCs were stimulated with PDGF for $24 \mathrm{~h}$ and abundance of LC3 was detected by Western blotting; B, C) Quantification of LC3 from panel A. ${ }^{*} \mathrm{p}<0.05$ vs control group, $\mathrm{n}=3$ per group; D) Measurements of autophagic flux: Bafilomycin A1 $(2 \mu \mathrm{M})$ was added to control or PDGF-stimulated cells $4 \mathrm{~h}$ prior to harvest. Accumulation of LC3-II was measured by Western blotting; E, F) Quantification of LC3 from panel D. ${ }^{*} p<0.05$ vs. the indicated group, $n=3$ per group. ( $n=3$ per group, ${ }^{\#} p<0.05$ vs baf or vs PDGF+baf, ${ }^{*} p<0.05$ vs Ctrl); and G-J) Effects of FGF-1 on proliferation, contractile protein abundance, and autophagic flux in VSMCs: panel J, comparison of VSMC proliferation when treated with FGF$1(25 \mathrm{ng} / \mathrm{ml})$ and PDGF-BB $(25 \mathrm{ng} / \mathrm{ml})$ for the indicated time. Cells were counted at the end of the treatment period using a hemocytometer. Panel $\mathbf{H}$, representative western blots of contractile proteins in cells treated with FGF-1 (0-50 ng/ml); Panel I, representative western blot of autophagic flux in untreated and FGF-1-treated cells. Note that bafilomycin $(2 \mu \mathrm{M})$ was added $4 \mathrm{~h}$ prior to harvesting cells. Panel $\mathrm{J}$, densitometry measurements of LC3 from panel $\mathrm{H}$. ${ }^{*} \mathrm{p}<0.05$ vs. control group, $n=$ 3 per group. 
Figure 2
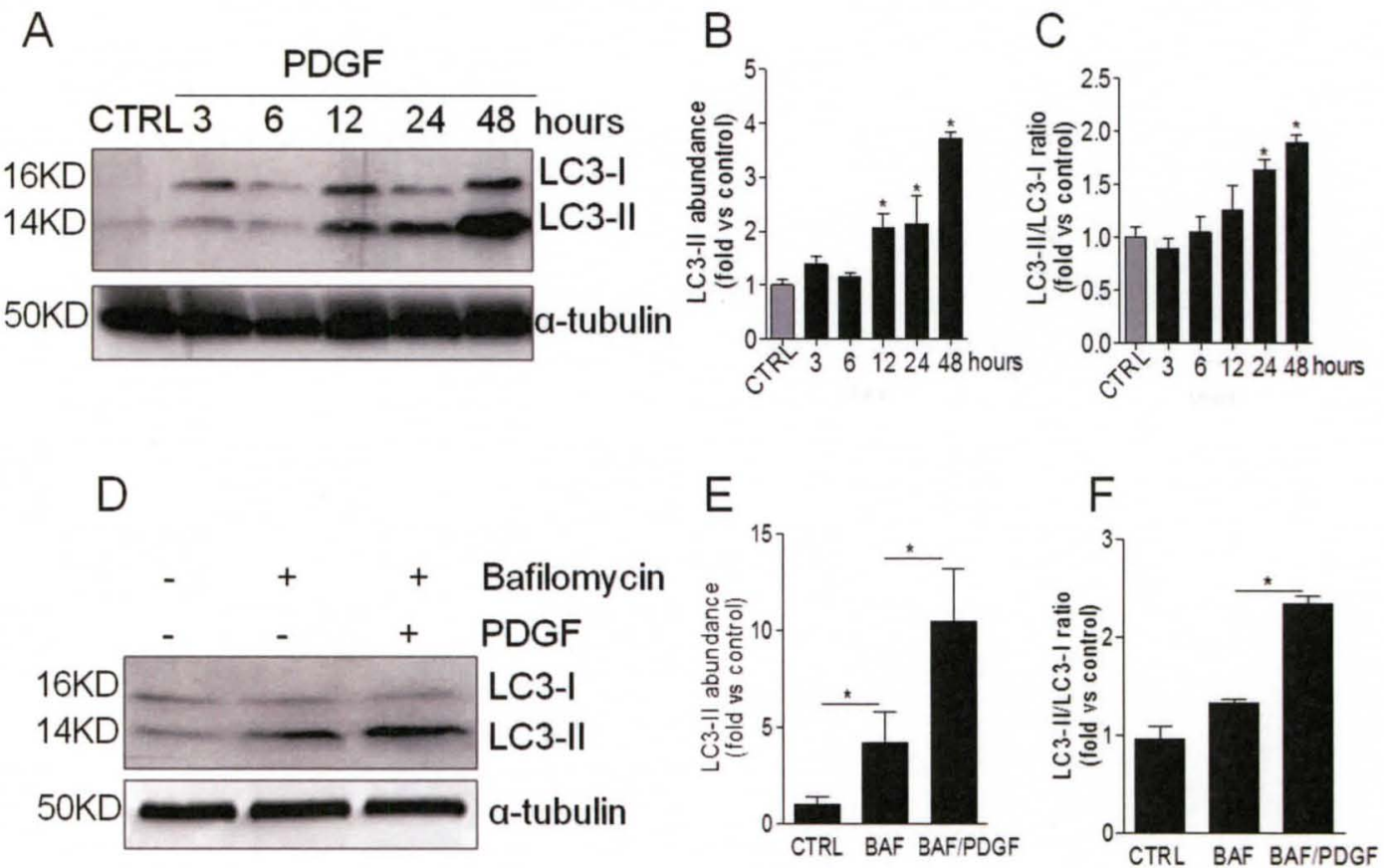

G

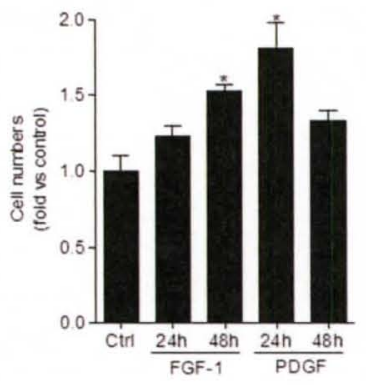

I

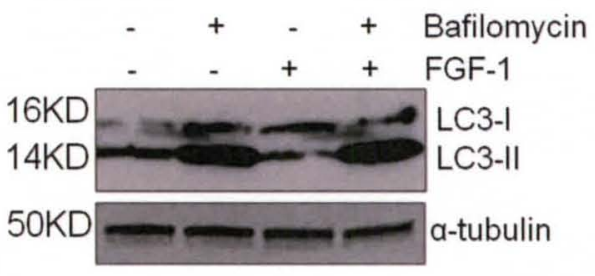

$\mathrm{H}$

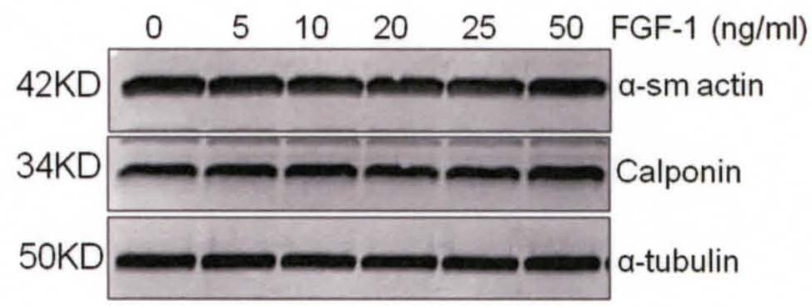

J

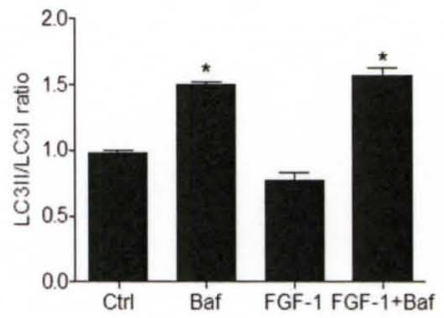


PDGF-induced autophagy is required for phenotype switching: To examine whether autophagy plays a role in VSMC phenotype switching, we pretreated VSMCs with the pharmacological inhibitor of autophagy, 3-methyladenine (3MA)[171], prior to stimulating the cells with PDGF. As shown in Fig. 4A and B, 3MA decreased GFP-LC3 puncta formation by $50 \%$. This inhibition of autophagy was associated with partial stabilization of the contractile markers calponin and aSMA (Fig. 4A-D) and inhibition of smooth muscle cell proliferation (Fig. 4E). Because of potential, off-target effects of 3-MA [172-174] and the relatively high concentrations of the compound needed to inhibit the autophagic machinery, we tested whether spautin-1, a more specific inhibitor of autophagy, would affect VSMC phenotype. Spautin-1 has been recently shown to prevent autophagy by inhibiting the deubiquitinases USP10 and USP13, which leads to beclin 1 degradation (Fig. 5A) [175]. Pretreatment of VSMCs with spautin-1 (10 $\mu \mathrm{M})$ prevented PDGF-induced disorganization of actin filaments (Fig. 5B). Western blot analysis demonstrated that inhibition of autophagy with spautin-1 prevented increases in LC3-II and losses of calponin and $\alpha$-SMA after PDGF exposure (Fig. 5C-F). Spautin treatment also prevented VSMC hyperproliferation (Fig. 5G).

To determine if the proteasome plays a role in regulating VSMC phenotype switching, we treated cells with the proteasome inhibitor epoxomicin [176] prior to PDGF treatment and examined contractile protein expression. Although epoxomicin treatment inhibited proteasomal activity, as evidence by the increased abundance of ubiquitinated proteins (Fig. 6A, B), the loss of contractile proteins 
due to PDGF treatment remained largely unaffected (Fig. 6C-E). Collectively, these results suggest that autophagy, and not proteasomal activity, is required for phenotypic changes in PDGF-stimulated VSMCs.

Synthetic VSMCs are resistant to aldehyde-induced cell death: Lesions in atherosclerotic and restenotic vessels are characterized by increased amounts of proteins modified by lipid peroxidation products such as 4-hydroxy-trans-2-nonenal (4-HNE) [177-178]. Because previous findings suggest that autophagy prevents toxicity due to 4-HNE [133], we hypothesized that synthetic VSMCs having higher autophagic flux might be protected from 4-HNE-induced cell death. This could be important because synthetic VSMCs are the abundant phenotype found in vascular lesions; hence, their survival may be impacted by their ability to handle oxidative insults. Indeed, as shown in Fig. 7A, cell viability of synthetic VSMCs challenged with HNE was significantly enhanced compared with HNE-challenged contractile VSMCs $(p<0.05)$. Consistent with the role of autophagy in clearing oxidatively damaged proteins $[133,179]$, synthetic VSMCs resisted accumulation of proteinHNE adducts due to exogenous HNE exposure (Fig. 7B and C). Inhibiting autophagy with spautin-1 promoted accumulation of HNE-damaged proteins (Fig. $7 \mathrm{D}$ and $\mathrm{E})$. These results suggest that PDGF-induced autophagy may be important for promoting the survival of VSMCs found in diseased vessels. 
Figure 3. PDGF-BB increases autophagosome formation and promotes extensive vacuolization. Microscopic evaluation of autophagy and VSMC ultrastructure: A) Representative confocal images of VSMCs transfected with AdGFP ( $\mathrm{i}$ and ii) or Ad-GFP-LC3 (iii, iv, v, and iv) treated without or with PDGF and/or bafilomycin; B) Quantification of puncta in panel B (> 20 cells counted per field in each group, ${ }^{*} p<0.01$ vs. the indicated groups; $\left.C-E\right)$ Representative transmission electron micrographs of cells treated with vehicle (panel C) or PDGF (panel D) for 48 h. E) Magnified image of boxed area in panel B. Double membrane-bound structures identified as autophagosomes in panels $D$ and $E$ are indicated by the red arrows. $\mathrm{N}=$ nucleus, $\mathrm{M}=$ mitochondria, $\mathrm{V}=$ =vacuoles. Magnification $=\times 7100 \mathrm{~F}$ ) Quantification of autophagosomes in images from control and PDGF treatment groups. ${ }^{*} p<0.05$ vs control. 
Figure 3

A

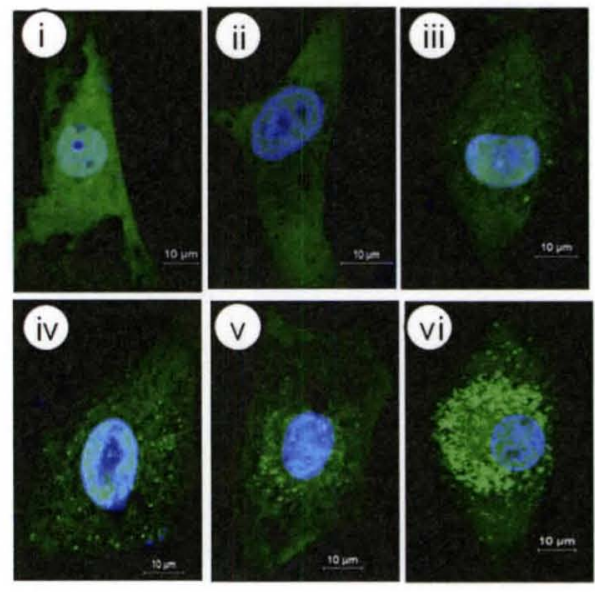

B

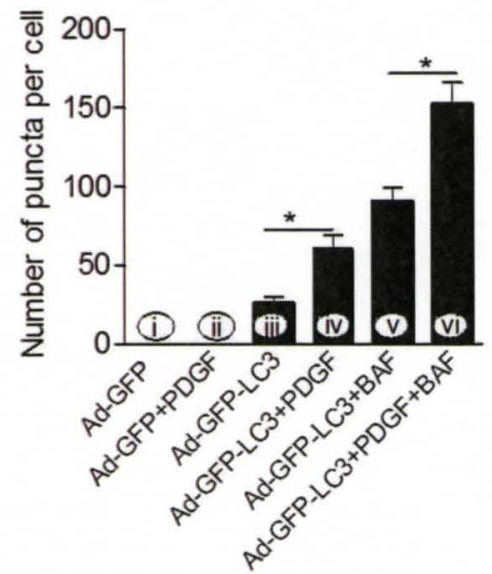

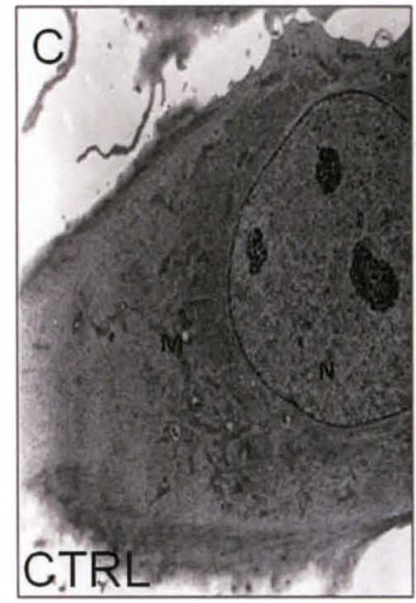
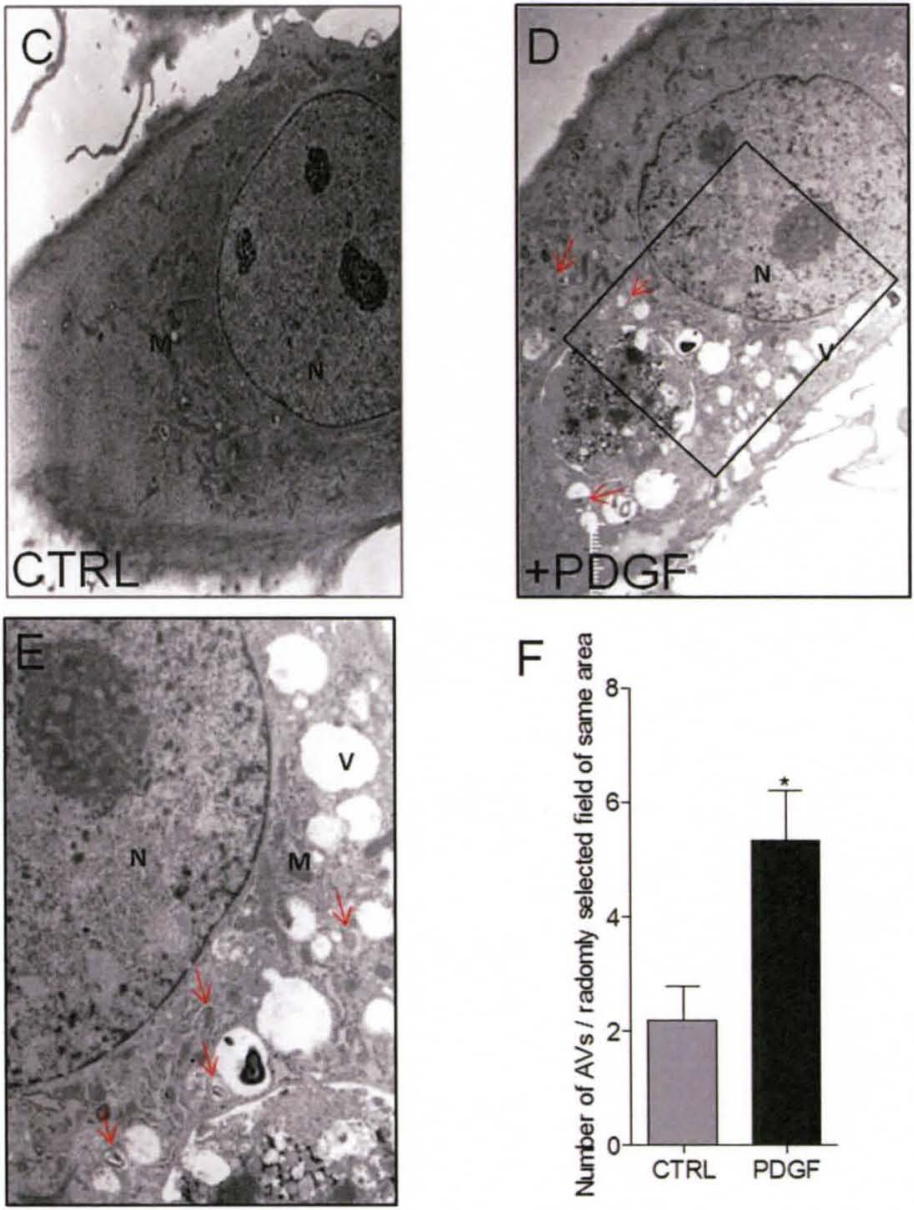


\section{Discussion}

The present study demonstrates autophagy as a novel mechanism regulating VSMC phenotype. We found that PDGF, which promotes the development of the synthetic VSMC phenotype, is a robust inducer of autophagy and that pharmacological inhibition by two structurally-unrelated inhibitors of autophagy blocked the degradation of calponin and a-sm-actin and prevented hyperproliferation and the transition to the synthetic phenotype. We also found that the synthetic phenotype was more resistant to the electrophilic stress commonly encountered in diseased vessels, and this was associated with an increased ability to remove proteins damaged or modified by electrophiles. These findings uncover PDGF-mediated autophagy as an important regulator of VSMC phenotype.

Previous studies have identified numerous factors that regulate phenotype plasticity in VSMCs. For example, contractile agonists, reactive oxygen species and extracellular matrix components have been shown to regulate the expression of contractile genes [53-54, 180-184]. Conversely, growth factors such as PDGF and oxidized phospholipids are known to favor the synthetic phenotype $[50,61,66$, 185]. Several studies have elucidated molecular mechanisms governing the switch to the synthetic phenotype. The signaling pathways known to trigger the synthetic phenotype culminate in the displacement of myocardin, a coactivator of serum response factor, from the consensus CArG box upstream of smooth muscle cell 
marker genes [186]. PDGF is known to act through transcription factors such as KLF4 and Elk-1 as well as microRNAs (such as miR221 and miR222) leading to the displacement of myocardin and downregulation of VSMC contractile genes [ 92 , 124, 187-188]. Hence, most studies to date examined transcriptional and translational changes regulating phenotypic transition; few, however, have explored post-transcriptional and -translational mechanisms.

We posited that one limiting event to transition to the synthetic phenotype would be removal of the contractile apparatus. Degradation of contractile proteins would likely involve the proteasome, autophagy, or both. Interestingly, the proteasome has been shown to play a role in VSMC hyperplasia but not phenotype switching $[130,189]$; and paradoxically, inhibitors of the proteasome decrease markers of the contractile phenotype [165] resulting in reduced cell contractility [166]. In our studies, we found that inhibition of the proteasome with epoxomicin had little effect on contractile protein abundance, suggesting to us that autophagy could be the major proteolytic device employed by the cell to remove contractile proteins.

We found that PDGF induced a robust form of autophagy characterized by increased LC3-II abundance, augmented autophagic flux, and the formation of autophagosomes and vacuoles. The induction of autophagy was specific to PDGF: the mitogen FGF, while increasing proliferation to a similar extent as PDGF, did not induce autophagy. While factors such as TNF- $\alpha$ and osteopontin have been shown to promote autophagy in VSMCs [109, 190], there are 
surprisingly few reports of induction of autophagy by growth factors. In fibroblasts and cancer cells, connective tissue growth factor (CTGF) has been shown to activate autophagy and regulate proliferation [191], and transforming growth factor$\beta$ (TGF- $\beta$ ) activates autophagy in hepatocellular carcinoma cells [192]. In these cases, the induction of autophagy is usually associated with diminished proliferation; however, we found that autophagy may be important for PDGFmediated hyperproliferation, as inhibition of autophagy with 3-MA or spautin-1 prevented cellular proliferation induced by PDGF. We hypothesize that this may be due either to stabilization of contractile proteins, which might structurally hinder cell division, or to decreased generation of metabolic building blocks, e.g., amino acids, which would be a natural consequence of enhanced autophagic activity and could be used to supply energy requirements for increased proliferation.

It remains unclear why rapamycin, which is an inhibitor of mTOR and activator of autophagy, promotes the contractile phenotype in VSMCs $[83,193]$ while PDGFmediated autophagy induces the synthetic phenotype. It is likely that the form of autophagy induced by PDGF is uniquely poised to degrade contractile proteins and is tailored to regulate the phenotype switch.

In addition to contractile proteins, autophagy has been shown to be important in preventing accumulation of damaged or aggregated proteins $[133,179,194]$. We found that the increase in autophagic flux in PDGF-treated VSMCs was sufficient to decrease cell death and the abundance of protein-HNE adducts after an 
aldehyde challenge, suggesting that synthetic VSMCs may be better able to tolerate the high levels of oxidant stress occurring in vascular lesions. This may be an important feature of synthetic cells, as it may regulate the survival of VSMCs in the fibrous cap, which, at least in part, is responsible for maintaining plaque stability [195]. Interestingly, the form of autophagy induced by PDGF appears to be functionally different from what has been described to occur with other stimuli. For example, TNF- $\alpha$ and IGF-1 were shown to increase autophagy in VSMCs, yet promote an autophagic form of cell death [190]. That PDGF promotes autophagy, cell survival, and increases the formation of extracellular matrix therefore suggests that it safeguards plaque-resident VSMCs against oxidative injury while potentially increasing the stability of lipid-laden atherosclerotic plaques.

Although the non-specific effects of pharmacological inhibitors cannot be rigorously ruled out, our observation that two structurally different inhibitors that target different components of the autophagic machinery were effective at stabilizing calponin and $\alpha$-SMA support the view that autophagy is required for PDGFinduced phenotype conversion. Nevertheless, spautin was more robust in stabilizing the contractile VSMC phenotype, and it completely prevented the hyperproliferation of VSMCs after PDGF exposure. The inhibition of autophagy with spautin did not affect PDGF signaling through Akt and Erk, further suggesting that inhibition of phenotype switching was due to stabilization of the contractile apparatus (Figure 8). These results suggest that spautin might be a useful 
therapeutic agent for preventing abnormal proliferation of smooth muscle cells in vascular injury or restenosis.

In summary, the present study identifies a new mechanism by which autophagy is activated and shows that autophagy is critical for attaining a synthetic VSMC phenotype and for increasing resistance to oxidative stress. Our observations also suggest that therapies targeting autophagy might be useful in preventing aberrant smooth muscle cell growth, but that such interventions may have deleterious effects on synthetic smooth muscle cell survival under conditions of oxidative stress. Future studies are required to identify the detailed molecular mechanism(s) by which PDGF activates the autophagic program and to test how modulating PDGF-mediated autophagy affects the progression of vascular disease. 
Figure 4. Inhibition of autophagy prevents PDGF-induced phenotype switching. Measurements of autophagy and contractile phenotype in cells treated with autophagy inhibitors: A) Representative confocal images of VSMCs treated with PDGF in the absence or presence of 3-MA (10 mM). B) Quantification of fluorescent puncta from panel A. ${ }^{*} p<0.03, n=10$ per group. C) Western analysis of contractile protein expression. VSMCs were serum-starved for $24 \mathrm{~h}$ and then stimulated with PDGF $(20 \mathrm{ng} / \mathrm{ml})$ for 48 hours. 3-MA (10 mM) was added 30 min prior to PDGF stimulation. D-E) Quantification of immunoblots represented in panel C. F) Cell proliferation in VSMCs: VSMCs were treated with vehicle or PDGF in the absence or presence of 3-MA. After $48 \mathrm{~h}$, the cells were trypsinized and counted using a hemocytometer. ${ }^{*} p<0.05$ vs. PDGF only and ${ }^{*} p<0.05$ vs non-PDGFtreated cells, $n=3$ per group. 


\section{Figure 4}

A
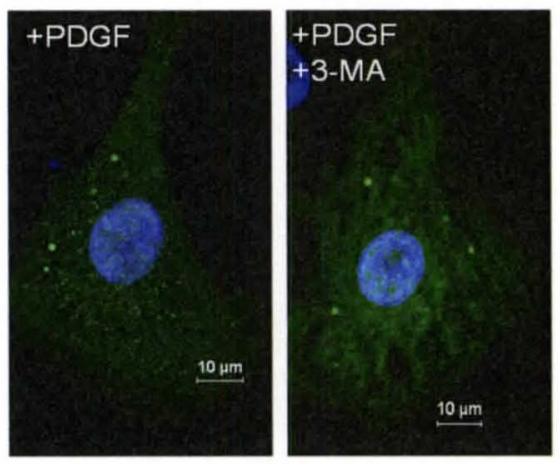

C

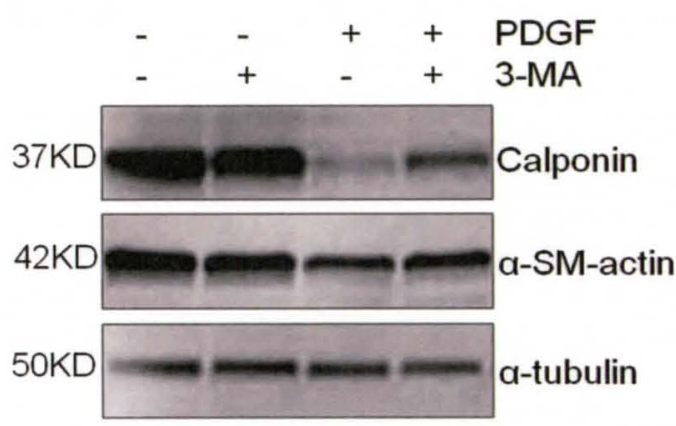

E

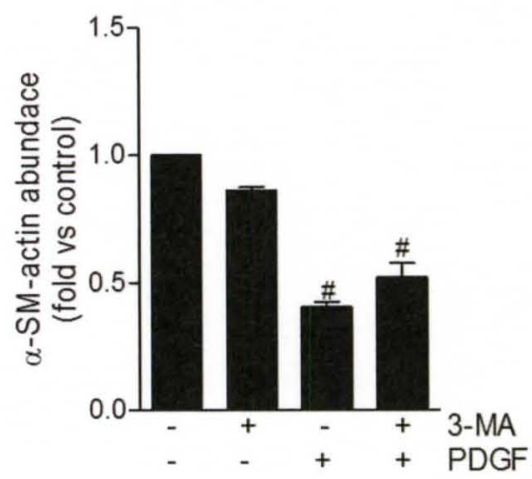

B
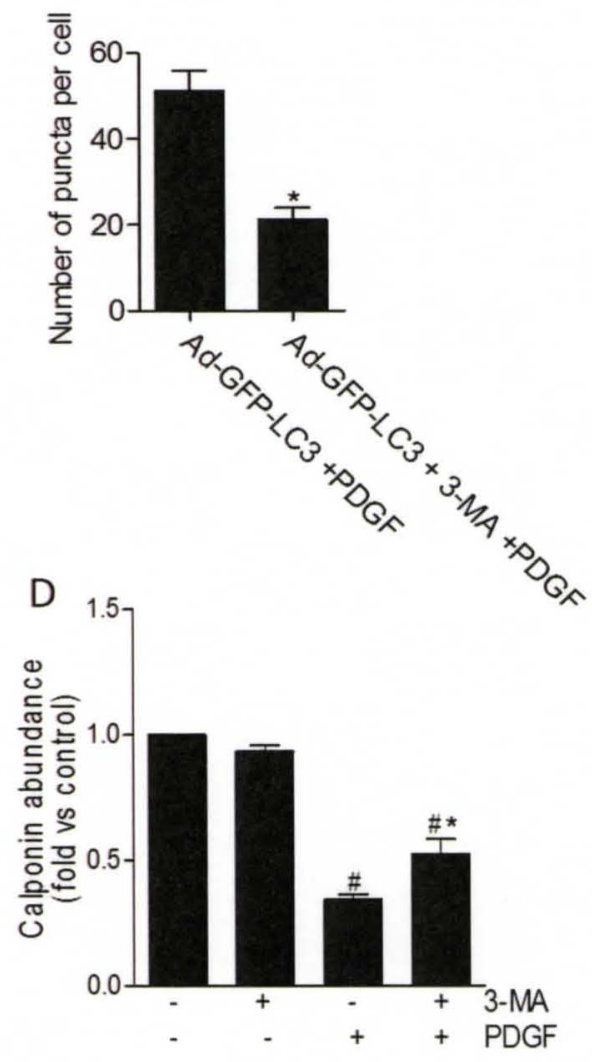

F

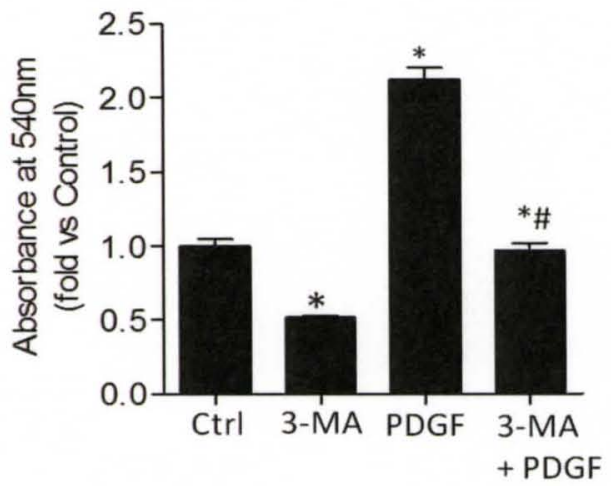


Figure 5. Spautin 1, an inhibitor of autophagy, prevents PDGF-induced phenotype switching. A) Mechanism of action of spautin 1: Spautin 1 promotes the degradation of beclin 1 through inhibition of USP10 and USP13 deubiquitinases; B) Representative confocal images VSMCs treated without or with PDGF in the absence or presence of spautin $1(10 \mu \mathrm{M})$. Scale bar $=10 \mu \mathrm{m} . \mathrm{C})$ Representative Western blots of LC3 and contractile proteins. D-F) Quantification of immunoblots from panel C. G) Cell proliferation in VSMCs: VSMCs were treated with vehicle or PDGF in the absence or presence of spautin-1. After $48 \mathrm{~h}$, the cells were trypsinized and counted using a hemocytometer. ${ }^{*} \mathrm{p}<0.05$ vs. CTRL; ${ }^{*} \mathrm{p}<0.05$ vs. PDGF group, $n=3$ per group. 
Figure 5

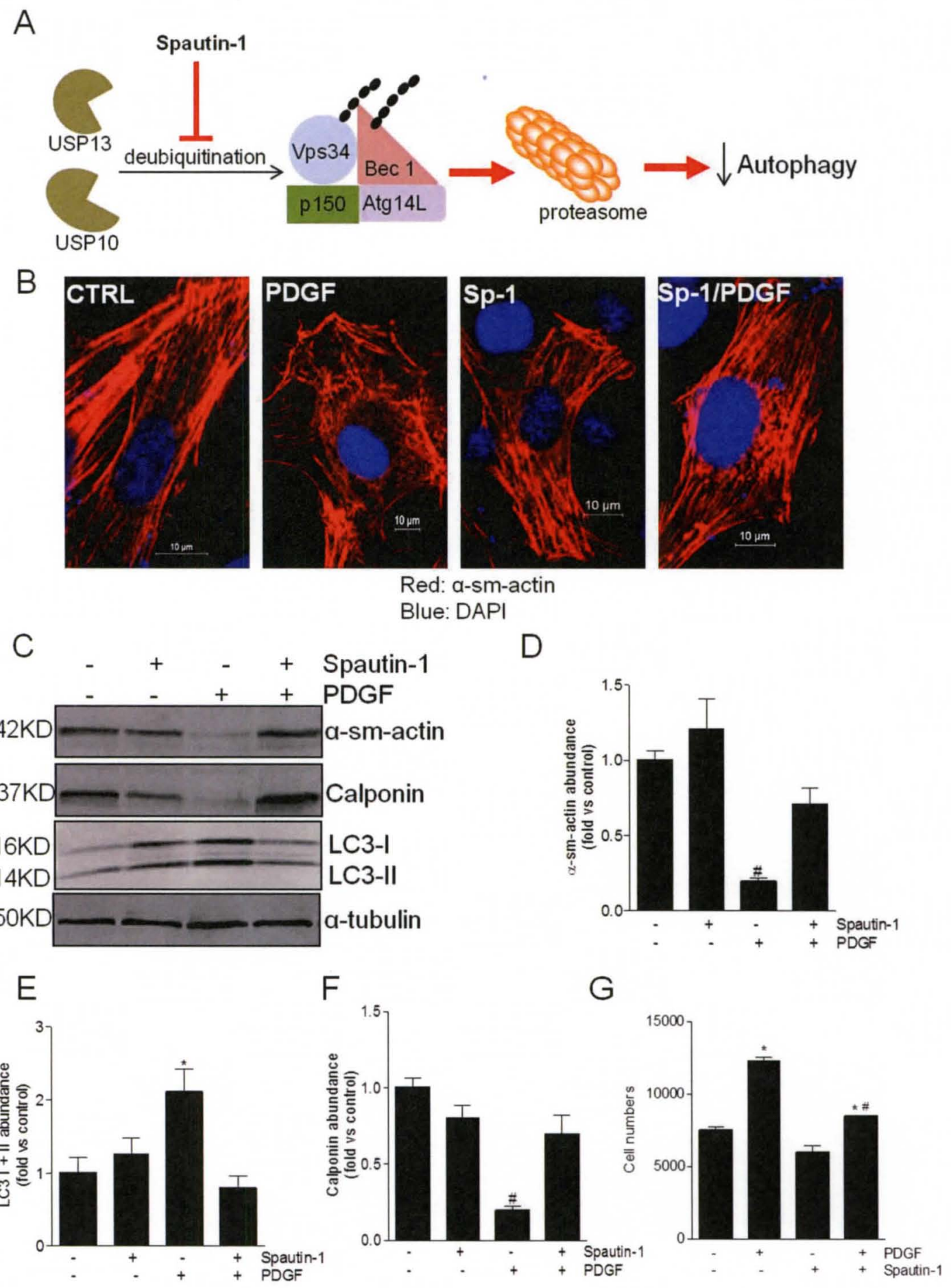


Figure 6. Inhibition of the proteasomal pathway does not inhibit PDGFinduced contractile protein degradation. Immunoblot analysis of ubiquitinated proteins and contractile proteins: A) Accumulation of ubiquitinated proteins after epoxomicin (100 nM) treatment; B) Quantification of $A$ ( $n=3$ per group, ${ }^{*} p<0.05$ vs control). C-E) Effects of epoxomicin on calponin and a-sm-actin degradation. C) Representative western blots. D-E) quantification of $C$ ( $n=3$ per group, ${ }^{*} p<0.05$ vs PDGF). 
Figure 6

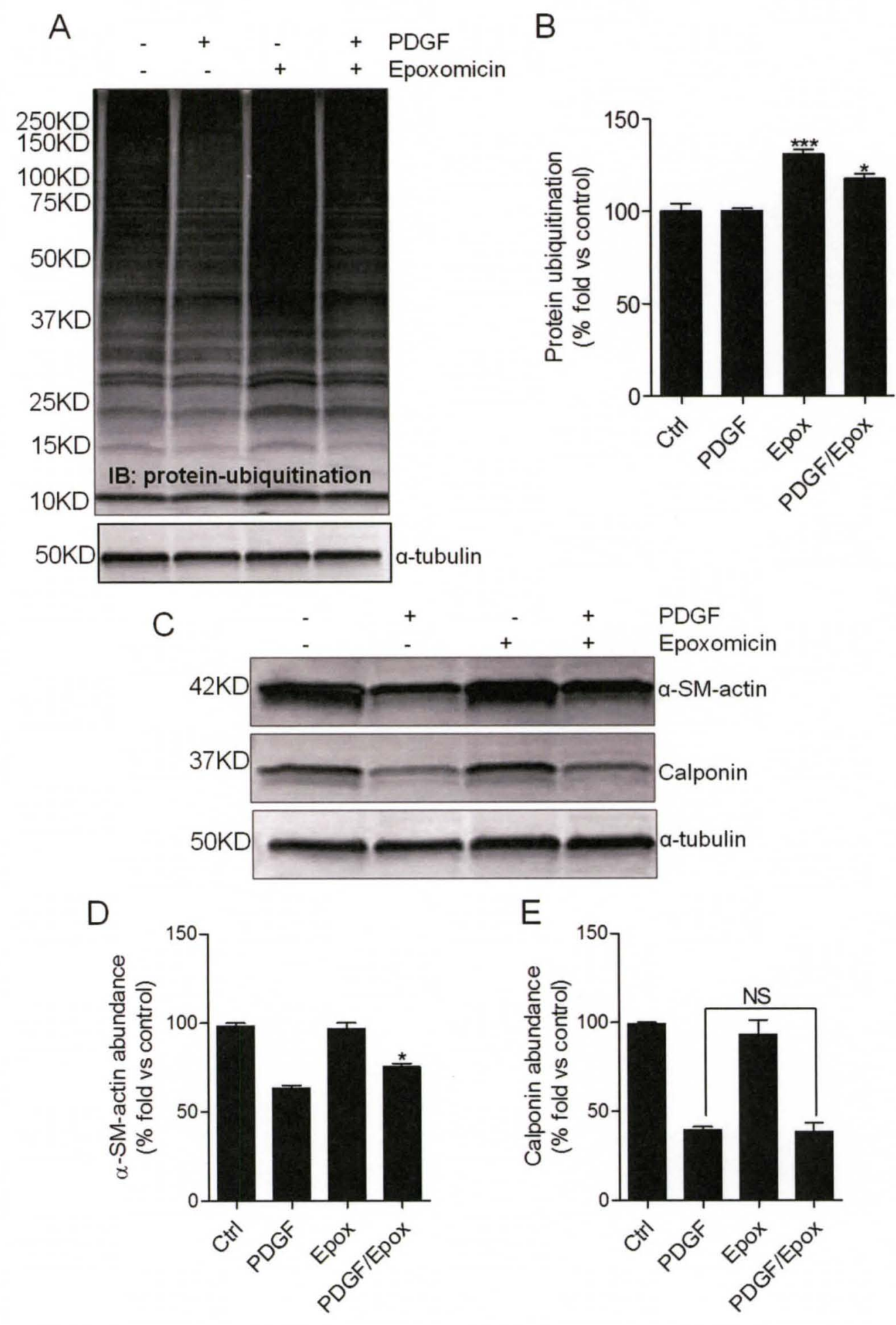


Figure 7. Synthetic VSMCs are resistant to HNE-induced toxicity. A) Cell viability in HNE-treated cells: VSMCs were treated with vehicle or PDGF for $48 \mathrm{~h}$ and then exposed to HNE $(50 \mu \mathrm{M})$ for $30 \mathrm{~min}$ in HBSS. The HNE-containing medium was removed and replaced with growth medium, and cell viability was assessed by LDH assay $16 \mathrm{~h}$ later. ${ }^{*} p<0.05$ vs. HNE, ${ }^{\&} p<0.05$ vs $C T R L, n=3$ per group. B, C) Protein-HNE adduct formation in contractile (control) and synthetic (PDGF-treated) cells: The cells were treated with HNE $(50 \mu \mathrm{M})$ for 30 min in HBSS. The medium was then replaced with growth medium and the cells were harvested after $3.5 \mathrm{~h}$ for examination of HNE adducts. Representative proteinHNE blots are shown in panel B and quantification is shown in panel C. ${ }^{*} p<0.005$ vs $\mathrm{HNE}, \mathrm{n}=3$ per treatment group. D) Representative immunoblots of protein-HNE adducts in contractile and synthetic VSMCs treated without (left panel) or with spautin-1 (right panel). E) Quantification of groups shown in panel D. All densitometric measurements were normalized to $\alpha$-tubulin. ${ }^{*} p<0.05, n=3$ per group. 
Figure 7
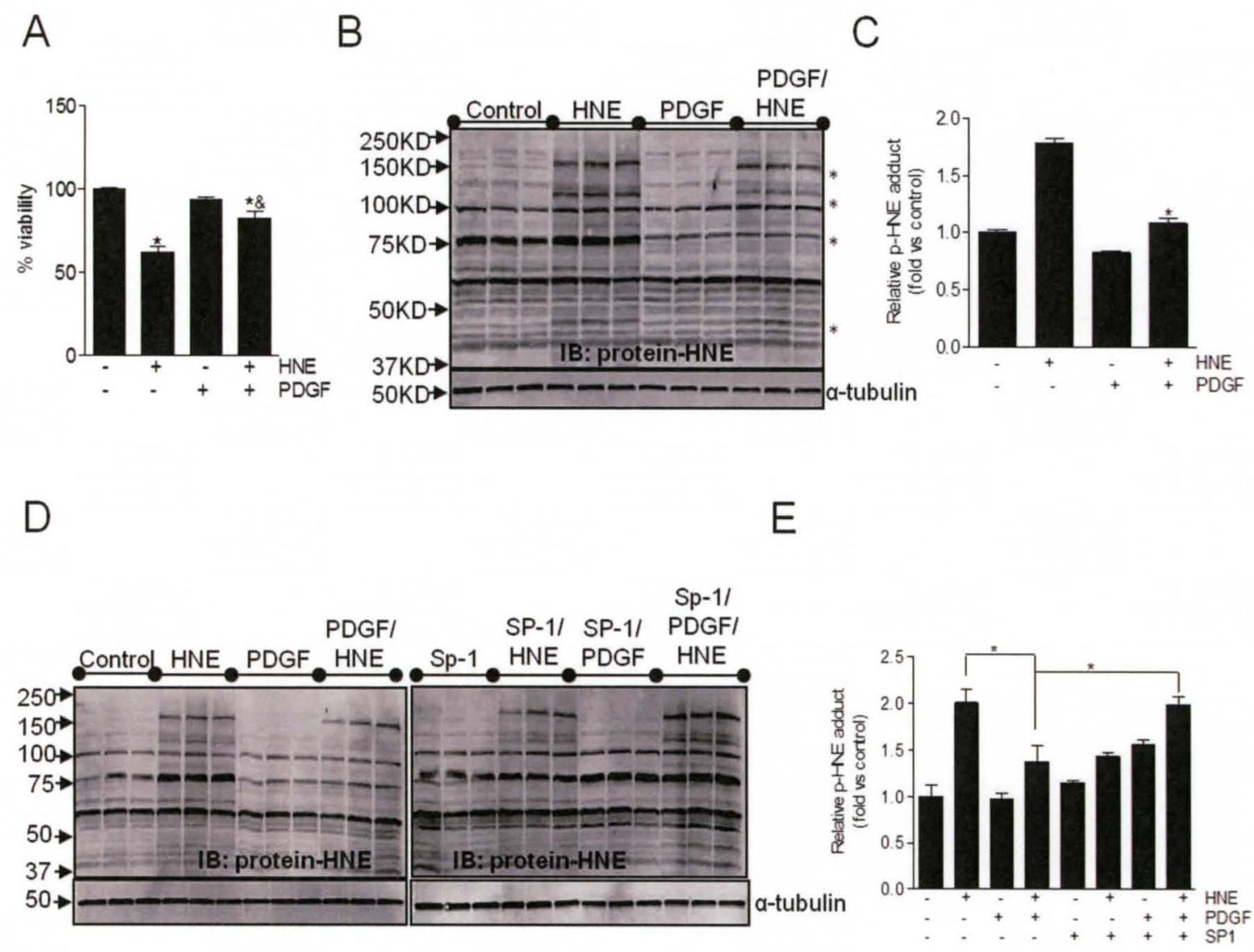
Figure 8. Spautin 1 does not interfere with PDGF-induced Akt or Erk1/2 signaling. A) Western blot analysis of Akt and Erk1/2 phosphorylation status: VSMCs were pretreated with spautin-1 for 30 min before PDGF stimulation at the indicated times. B, C) Quantification of $A\left(n=3,{ }^{*} p<0.05\right.$ vs 0 min). 
Figure 8

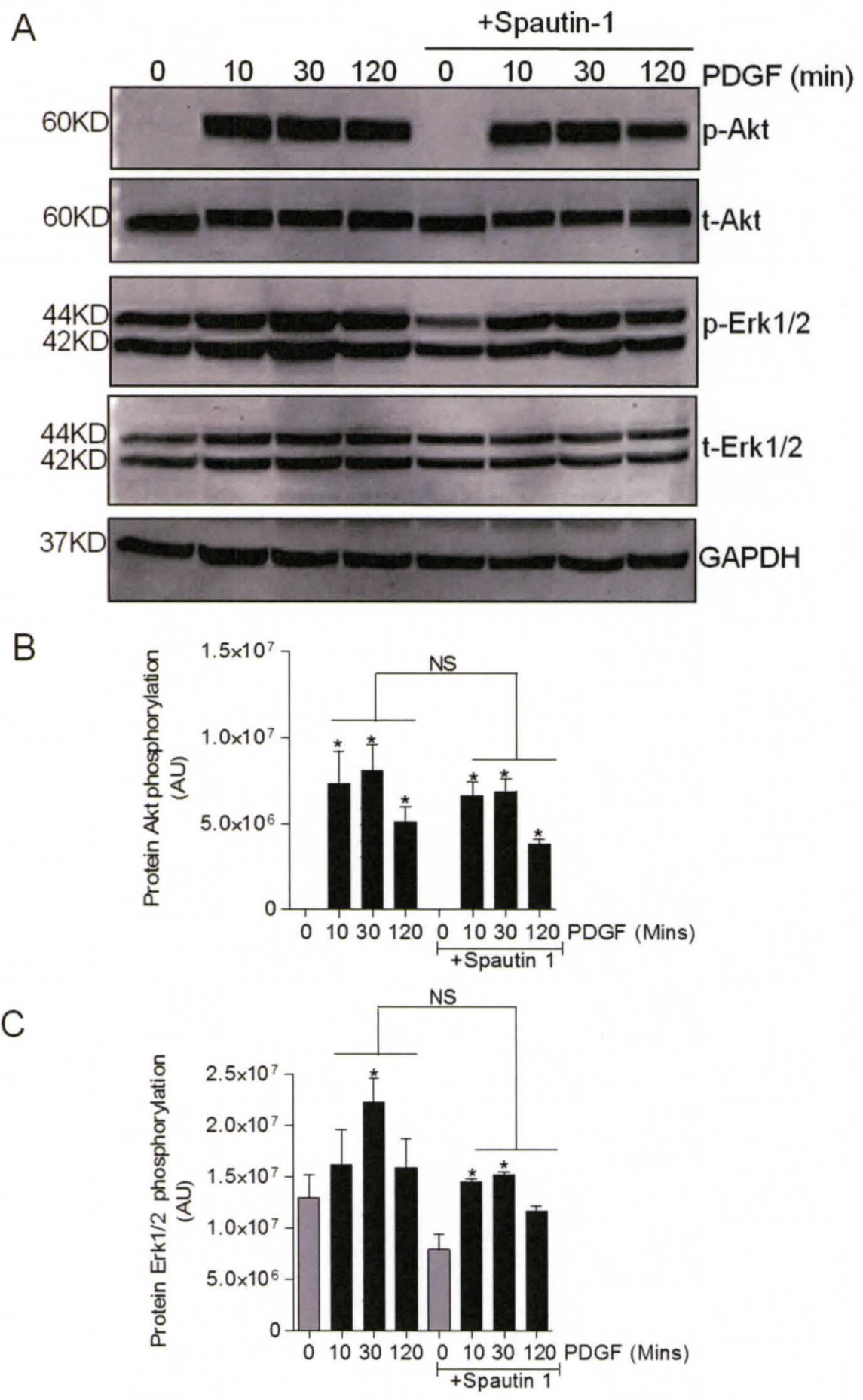




\section{CHAPTER III}

\section{REGULATION OF VASCULAR SMOOTH MUSCLE CELL PROLIFERATION AND PHENOTYPE SWITCHING BY O-GIcNAc}

\section{Introduction}

Glucose metabolism in VSMCs occurs via four major pathways: the glycolytic, the polyol, the hexosamine biosynthetic, and the pentose phosphate pathways. These pathways have been shown to regulate VSMC hyperplasia and function. For example, inhibition of aldose reductase, the rate-limiting enzyme in the polyol pathway, prevents VSMC proliferation induced by the cytokine TNF- $\alpha$ [196]; increased flux into the hexosamine biosynthetic pathway (HBP) has been shown to inhibit VSMC proliferation and reduce neointima formation after balloon injury [197]; increased glycolytic flux promotes PDGF-induced VSMC hyperplasia [198]; and, glucose-6-dehydrogenase (G6PD), the rate-limiting enzyme in the pentose phosphate pathway, has been shown to regulate VSMC contractility [199]. Thus, glucose metabolism is an important aspect of vascular complications involving the VSMC. Despite such findings, vascular proliferative diseases such as atherosclerosis continue to be major causes of death [200]. Therefore further understanding of glucose metabolism in the VSMC is needed to expose new avenues for clinical intervention. 
VSMC hyperproliferation is mediated by growth factors and environmental cues. Platelet derived growth factor BB (PDGF-BB or PDGF) is the most potent inducer of VSMC proliferation and it is released from platelets, macrophages, and endothelial cells during vessel injury [36]. PDGF stimulation of VSMCs causes increased secretion of cytokines, particularly osteopontin, a pro-proliferative cytokine which is important for VSMC migration and extracellular matrix formation during atherogenesis and restenosis [103]. The levels of osteopontin expression are regulated by transcription factors which are under the influence of cellular glucose levels [201]. This suggests that regulating glucose metabolism (or cellular glucose concentrations) could be important in regulating VSMC osteopontin expression and VSMC proliferation. In particular, the mechanisms by which glucose metabolism regulate osteopontin expression are unclear and how accessory pathways, such as the HBP, affect OPN expression and VSMC phenotype are understudied.

The hexosamine biosynthetic pathway (HBP) utilizes about $2-5 \%$ of glucose in mammalian cells [202], and this pathway has been implicated in VSMC neotintimal formation during vascular remodeling [203]. The enzyme, Lglutamine-D-fructose 6-phosphate amidotransferase (GFAT) is the regulatory enzyme mediating glucose flux into the HBP [204]. GFAT converts fructose-6phosphate to glucosamine-6-phosphate which, through a series of reactions is subsequently converted to uridine diphosphate $\mathrm{N}$-acetylglucosamine (UDPGIcNAc). UDP-GIcNAc is then used as a donor for the formation of O-linked-Nacetylglucosamine (O-GlcNAc) modified proteins as well as other proteoglycans, 
glycosamino glycans, and glycolipids [205]. O-GlcNAc modification regulates several biological processes such as apoptosis, signal transduction, transcription, translation, nuclear transport and protein degradation [206-213]. O-GlcNAcylation is also important in diseases such as cancer, diabetes and Alzheimer's disease [214-217]. To date, two key enzymes which perform kinase-like and phosphatase-like activities with respect to protein O-GIcNAc modification have been described: O-GIcNAc transferase (OGT) catalyses the transfer of the GIcNAc ( $\beta$-N-acetylglucosamine) moiety from UDP-GlcNAc to conserved serine/threonine residues of protein via an O-linkage [218]. Proteins which have been O-GIcNAc modified are converted back to their unmodified form through the action of O-GIcNAcase which removes the GIcNAc moiety to form UDP-GIcNAc [219]. Flux into the HBP controls levels of O-GlcNAcylated proteins, and OGT is very sensitive to levels of cellular UDP-GIcNAc [220]. In 1998, Nerlich et al [221] found an increase in GFAT expression under hyperglycemic conditions thereby suggesting a role of the HBP in diabetic vascular complications. Akimoto et al [222] later showed that high glucose caused elevated OGT expression leading to alteration of protein O-GIcNAc patterns. These studies suggest that the increase in VSMC hyperplasia observed under hyperglycemia is attributable to increased in glucose flux into the HBP. However, several studies have shown the contrary, for example, Slawson et al [223] showed that increasing levels of protein $O$ GICNAc modification using pharmacological inhibitors such as PUGNAc (which inhibits O-GIcNAcase) or through adenoviral gene over expression of OGT led to altered expression of cyclins and defects in mitotic progression. Subsequently, 
Xing et al [203] showed that increasing protein O-GlcNAc by glucosamine or PUGNAC treatment inhibited neointima formation after balloon injury of the rat carotid artery. This inhibition, Xing and colleagues suggest, was due to inhibition of cytokine expression after injury. However, the direct role of the HBP and OGICNAc protein modification on VSMC phenotype has not been assessed. Therefore, studies addressing the effects of glucose metabolic pathways such as glycolysis and the HBP on phenotype switching and hyperproliferation are needed.

In this study, we determined the effects of the GAPDH inhibitor, koningic acid $(\mathrm{KA})$, and the O-GICNAcase inhibitor, thiamet-g on PDGF-induced VSMC proliferation. These inhibitors have been demonstrated to be specific to their respective targets at low concentrations while producing minimal or no side effects [224-225]. Both KA and thiamet-g prevented PDGF-induced VSMC proliferation. KA inhibited glucose flux to glycolysis and inhibited osteopontin expression. These effects of KA resulted in inhibition of PDGF-induced proliferation. KA treatment led to a small but significant increase in levels of protein $\mathrm{O}-\mathrm{GlcNAc}$ formation thereby suggesting an antiproliferative role of the HBP. Indeed, Thiamet-g treatment, which caused increased protein O-GlcNAc formation, inhibited PDGF-induced osteopontin expression, and stalled the cells in the S-phase of the cell cycle. Expression of cell cycle proteins cyclin D1 and PCNA were inhibited in the presence of Thiamet-g. These findings reveal the importance of the glycolytic and the HBP pathways with respect to PDGFinduced VSMC proliferation and reveal two new inhibitors of VSMC proliferation 
that could potentially be developed for the treatment of vascular diseases such as atherosclerosis and restenosis.

\section{Experimental procedures}

Materials: Antibodies against $\alpha$-smooth muscle cell-actin ( $\alpha-S M A)$, calponin and $\alpha$-tubulin were purchased from Sigma-Aldrich (St. Louis, MO, USA). Antibodies against GAPDH, p-Akt/Akt and p-Erk1/2/Erk1/2 were purchased from Cell Signaling (Danvers, MA, USA). Antibodies against osteopontin, cyclin D1 and PCNA were purchased from Santa Cruz biotechnology (Santa Cruz, CA, USA). Antibody against protein O-GIcNAc was obtained from Covance (Princeton, NJ, USA). Recombinant rat PDGF was obtained from R\&D Biosystems (Minneapolis, MN, USA). HRP conjugated rabbit and mouse IgG secondary antibodies were obtained from Cell Signaling Technologies (Danvers, MA, USA). All primers used for real time PCR were designed using the primer express software from Applied Biosystems (Carlsbad, CA, USA) and then ordered from Integrated DNA Technologies (Coralville, IA, USA). Electrophoresis supplies were purchased from Bio-Rad (Hercules, CA, USA). $E C L^{\circledR}$ reagents were purchased from GE Healthcare (Pittsburgh, PA, USA). MTT reagent was obtained from Sigma Aldrich (St. Louis, MO, USA). Koningic acid was obtained from COSMO BIO CO LTD (Carlsbad, CA, USA). Thiamet-G was obtained from Cayman Chemical (Ann Arbor, MI, USA). The Cyquant proliferation assay kit was obtained from 
Invitrogen Life Technologies (Grand Island, NY, USA). Propidium lodide was purchased from eBioscince (San Diego, CA, USA).

Cell culture: Rat aortic smooth muscle cells (VSMCs) were isolated from the aortas of 6-week-old male Sprague-Dawley rats and grown in DMEM (Dulbecco's modified Eagle's medium; Life Technologies-Invitrogen) supplemented with $10 \%$ (v/v) FBS (fetal bovine serum; Atlanta Biologicals, Atlanta, GA, USA) and $0.1 \%$ streptomycin/penicillin. To ensure maintenance of the contractile phenotype, only cells between passages 2-7 were used. Cells were maintained in a humidified atmosphere of air and $5 \% \mathrm{CO}_{2}$ at $37^{\circ} \mathrm{C}$. At $\sim 70 \%$ confluency, VSMCs were serumstarved in DMEM containing $0.1 \%$ FBS for $24 \mathrm{~h}$. After desired treatments, cells were rinsed twice with phosphate-buffered saline and then lysed in a protein lysis buffer containing $25 \mathrm{mM}$ HEPES, $1 \mathrm{mM}$ EDTA, $1 \mathrm{mM}$ EGTA, $0.1 \%$ SDS, $1 \%$ NP40, and $1 \mathrm{X}$ protease and phosphatase inhibitors. The Lowry DC assay (Biorad, City, St., USA) was used for measuring protein concentration of crude cell extracts. Messenger RNA isolation and real-time PCR: Messenger RNA was isolated from RASMCs using TRIZOL reagent (Invitrogen) and the concentration was determined by measuring absorbance at $260 \mathrm{~nm}$ using a Nanodrop spectrophotometer (Thermo scientific). A $20 \mu \mathrm{l}$ reverse transcription reaction mixture containing $1 \mu \mathrm{g}$ mRNA, 10 units AMV reverse transcriptase, $0.4 \mu \mathrm{M}$ poly $\mathrm{T}$ primer (dT18), $0.2 \mathrm{mM}$ dNTPs, and 20 units RNasin (Promega) was subjected to complimentary DNA (cDNA) synthesis in a thermal cycler (BioRad). Two microliters of cDNA was then used for amplification of the gene of interest by real time PCR using SYBR green (VWR, Radnor, PA, USA). 
Western blotting: Approximately $0.5-25 \mu \mathrm{g}$ of crude cell protein was applied to each lane of a $10.5-14 \%$ Bis-Tris- $\mathrm{HCl}$ gel and electroblotted onto a PVDF membrane. The membrane was then incubated overnight at $4^{\circ} \mathrm{C}$ using appropriate dilutions of primary antibodies. PVDF membranes were then incubated at room temperature with horseradish peroxidase-conjugated secondary antibodies. Immunoreactive bands were detected using a typhoon scanner (SA Biosciences, Valencia, CA, USA) after exposure to $\mathrm{ECL}$ detection reagent. Bands intensity was quantified by using the TotalLab TL120 software.

For protein O-GlcNAc Western blots: VSMCs were treated with PUGNAc or thiamet-g, inhibitors of O-GlcNAcase, or vehicle $(0.1 \%$ ethanol) for overnight and then treated with PDGF for $48 \mathrm{~h}$. Total cell lysate was prepared as described above. After transfer of proteins onto PVDF, membrane was immediately put in methanol for $1 \mathrm{~min}$ and then allowed to dry at RT for $2 \mathrm{~h}$. After drying, membrane was incubated with 1:1000 dilution of anti-protein O-GlcNAc antibody (diluted in PBS-casein; cat no. 161-0783, Bio-Rad, Hercules, CA, USA) for overnight. Membrane was then washed $3 \times 10$ mins each and then incubated with appropriate secondary antibody (diluted in PBS-casein) for $1 \mathrm{~h}$ at RT. After second incubation, membrane was imaged using the Typhoon scanner.

\section{Measurement of cellular energetics using the XF24 extracellular flux} analyser: Cellular energetics was measured in intact VSMCs using a Seahorse Bioscience XF24 extracellular flux analyser. Approximately 40,000 cells were seeded in wells of a 24-well XF plate. Cells were then serum starved in $0.1 \% F B S$ DMEM for $24 \mathrm{~h}$. After serum starvation the media was replaced with phenol red 
free DMEM containing $1 \mathrm{mM}$ pyruvate, $5 \mathrm{mM}$ glucose and $3.97 \mathrm{mM}$ Glutamax-I. After equilibration in phenol red free DMEM, PDGF was then injected into cells and their extracellular acidification rates (ECARs) measured. Oligomycin was later injected to obtain the maximal glycolytic capacity of the cells. Koningic acid (KA) was added to inhibit glycolysis. Data obtained were normalized to the amount of protein present in the well of the microplate. For normalization, cells were lysed in the 24-well XF plates using $20 \mu \mathrm{l} /$ well of protein lysis buffer. Protein concentration was then measured using the Lowry's method.

Cell cycle studies with propidium iodide: VSMCs were harvested after 3 min trypsin incubation, washed with phosphate buffered saline (PBS) and cold $70 \%$ ethanol added to the cells. VSMCs were then fixed by incubating for 1 hour at $4^{\circ} \mathrm{C}$. After fixing, cells were sedimented at $500 \times \mathrm{g}$ for 5 mins and the supernatant

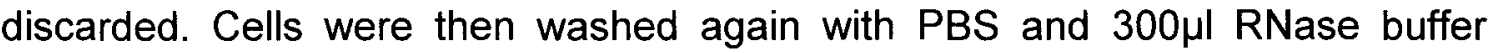
containing $5 \mu$ l propidium iodide added after all residual PBS has been discarded. Cells were then mixed and incubated at room temperature for 45 mins after which they were analyzed using a C6 Accuri flow cytometer.

Cell viability assays: VSMCs were serum starved for $24 \mathrm{~h}$ and then treated without or with PDGF $(20 \mathrm{ng} / \mathrm{ml})$ for $48 \mathrm{~h}$. After PDGF treatment, cells were exposed to $50 \mu \mathrm{M}$ HNE in HBSS for $30 \mathrm{~min}$ and then replaced with $10 \%$ FBS/DMEM media. After $16 \mathrm{~h}$, the medium was collected and lysates were prepared by scraping cells in $100 \mu$ of PBS containing $0.1 \%$ Triton X-100. LDH activity was measured spectrophotometrically for $4 \mathrm{~min}$ at $37^{\circ} \mathrm{C}$ by monitoring decrease in $\mathrm{NADH}$ absorbance at $340 \mathrm{~nm}$. Cytotoxicity was then estimated by 
dividing the change in absorbance of the medium by the values for combined $\mathrm{LDH}$ activity in the medium and the lysate as follows; cytotoxicity $=\left[\left(\mathrm{Abs}_{340}\right.\right.$ media * dilution factor) / ( Abs $_{340}$ Media * dilution factor $)+($ Abs lysate * dilution factor)] * 100. The values were then normalized relative to the control, which represented $100 \%$ cell viability.

For cytotoxicity assays, after desired treatment, cells were incubated in a solution of $0.2 \mathrm{mg} / \mathrm{ml} \mathrm{MTT}$ reagent for $2-4 \mathrm{~h}$ at $37^{\circ} \mathrm{C}$ in an incubator containing $5 \% \mathrm{CO}_{2}$. After incubation, MTT reagent was then aspirated and the purple crystals at the bottom of the plate dissolved in DMSO. Absorbance at $540 \mathrm{~nm}$ was then measured using a microplate reader.

Statistical analysis: Data are mean \pm SEM. Multiple groups were compared using one-way ANOVA, followed by Bonferroni post-tests. Unpaired Student's $t$-test was used for direct comparisons. A p-value $<0.05$ was considered significant. 


\section{Results}

PDGF induces expression of osteopontin in cultured VSMCs: To determine the effects of PDGF on glucose metabolism in isolated VSMCs, we first measured changes in proliferation and osteopontin expression on VSMCs isolated from rat aortas. For this, VSMCs were serum starved for $24 \mathrm{~h}$ in $0.1 \%$ FBS and then stimulated with PDGF $(20 \mathrm{ng} / \mathrm{ml})$ for $24 \mathrm{~h}$. Consistent with previous findings, PDGF induced about a 2.5 -fold increase in osteopontin protein abundance, increased proliferation by 2 -fold as measured by counting cell number or measuring DNA content (Figure 9A and B). The increase in cell proliferation was accompanied with 1.7- and 2.3-fold increases in the expression of cell cycle regulatory proteins such as cyclin D1 and PCNA, respectively (Figure $9 \mathrm{C}$ and $\mathrm{D}$ ).

PDGF increases glycolytic flux in VSMCs: To determine the effects of PDGF on glycolytic flux, we serum starved VSMCs for $24 \mathrm{~h}$ and then stimulated serum starved cells with different concentrations of PDGF. As shown in Figure 10, PDGF at a concentration of $25 \mathrm{ng} / \mathrm{ml}$ increased basal and maximal glycolytic flux with an insignificant increase in the glycolytic reserve capacity. Koningic acid completely inhibited glycolytic flux in VSMCs. 
Figure 9. PDGF-BB induces VSMC proliferation and expression of osteopontin. A and B) Measurement of cell proliferation after PDGF stimulation. Cell proliferation measured by counting cell numbers $(A)$ and by measurement of cellular DNA content using the cyquant fluorescent dye (B), $n=6,{ }^{*} p<0.01$ vs. control. C and D) Expression of cell cycle proteins and osteopontin after PDGF stimulation. (C) Western blot of cyclin D1, PCNA and osteopontin after PDGF stimulation. (D) Quantification of $C, n=3,{ }^{*} p<0.05$ vs. control. 
Figure 9

A

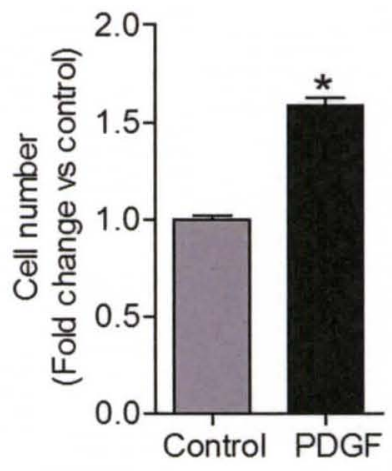

C

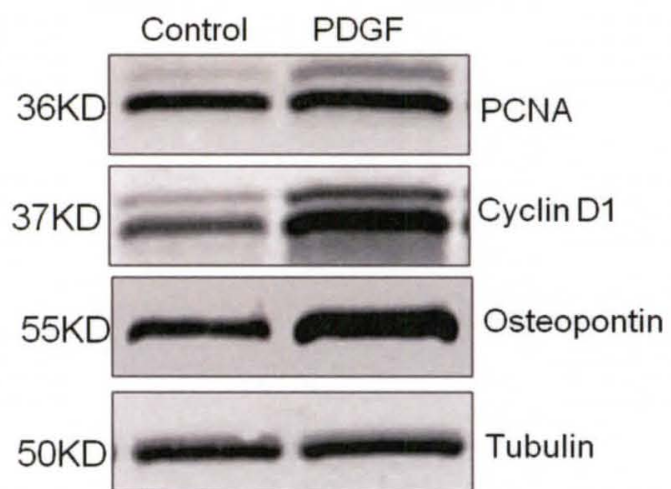

B

D
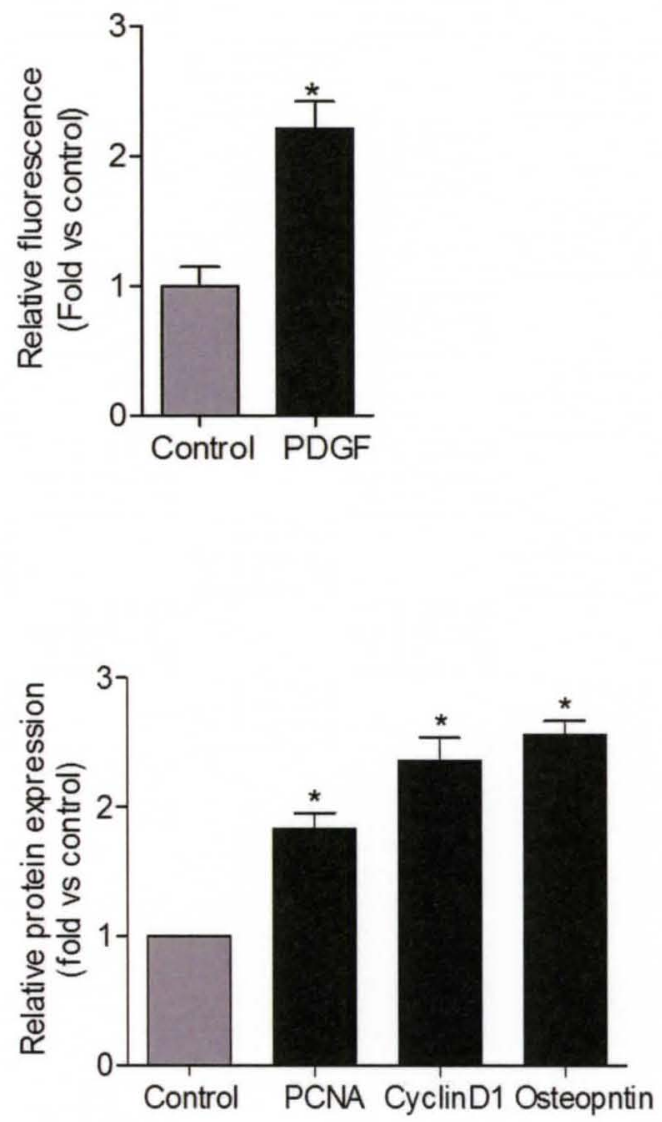
PDGF-induced osteopontin expression is dependent on glycolysis: To determine whether the increase in glycolytic flux caused by PDGF stimulation is important for proliferation and expression of the key synthetic gene osteopontin, we stimulated VSMCs with PDGF in the presence of koningic acid. As shown in Figures $11 \mathrm{~A}-\mathrm{C}$, koningic acid inhibited PDGF-induced VSMC proliferation. PDGF stimulation of VSMCs caused significant increases in mRNA (14-fold increase) and protein levels (2.5-fold increase) of osteopontin and these were inhibited by koningic acid (Figures 11D-F). These findings are all consistent with published data implicating the role of osteopontin in VSMC proliferation [201] and are novel because it is the first time that it is being directly shown that glycolytic flux is important in PDGF induced VSMC osteopontin expression.

Koningic acid treatment increases protein-O-GICNAC formation: We treated VSMCs with different concentrations of KA and monitored protein O-GICNAC formation. We found that protein O-GIcNAc formation was increased (Figure 12). This suggested to us that increased protein O-GlcNAc formation might be a unique mechanism regulating OPN expression and VSMC proliferation. 
Figure 10. PDGF-BB induces increase in glycolytic flux. A) Extracellular flux analysis after PDGF stimulation. B) Quantification of basal and maximal ECARs after PDGF stimulation ( $n=5,{ }^{*} p<0.05$ vs. control). 
Figure 10

A
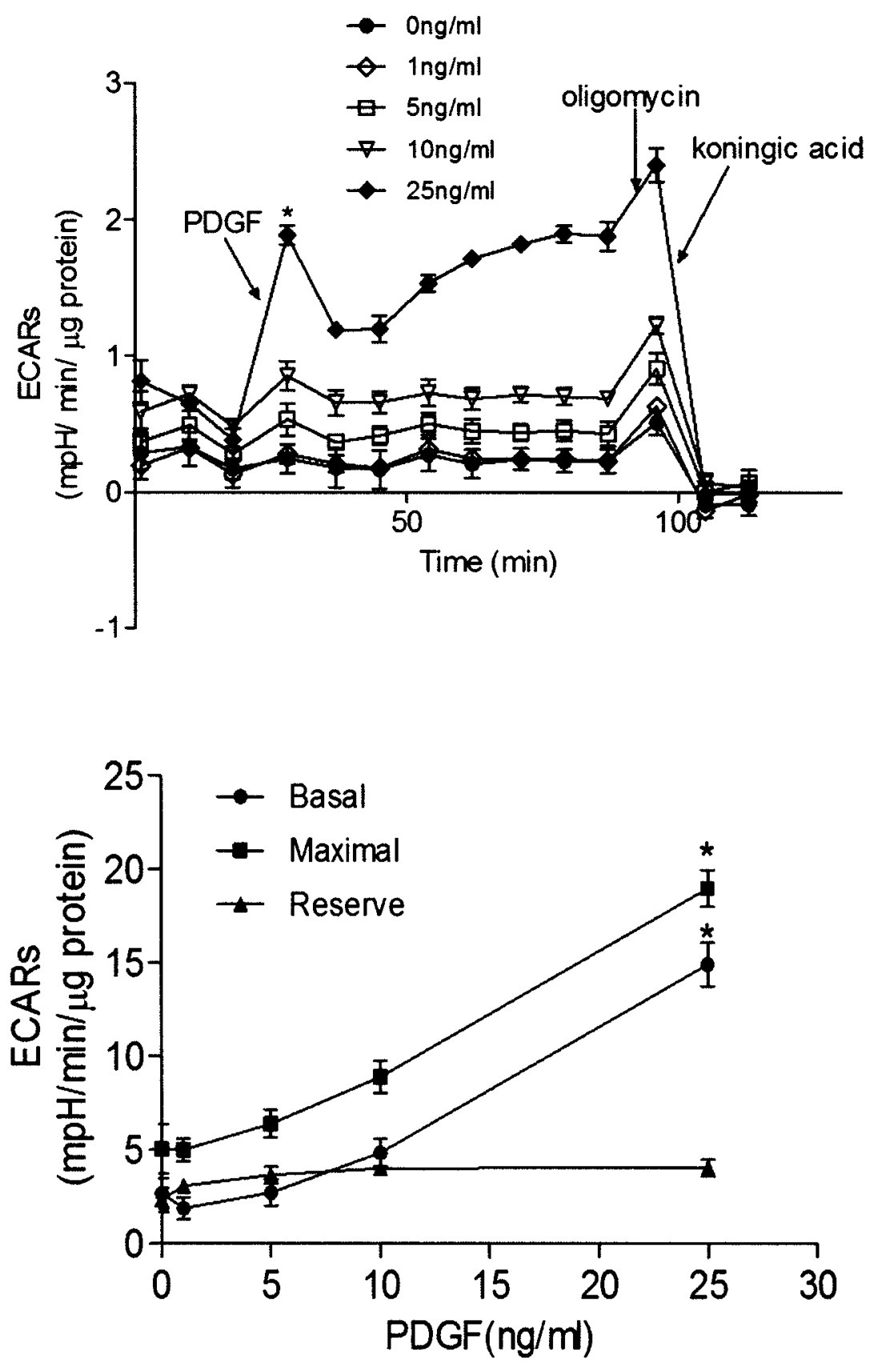


\section{Protein O-GICNAC regulates VSMC proliferation and osteopontin}

expression: Because changes in protein O-GIcNAc were observed after PDGF and KA stimulation, we further studied the role of increased O-GIcNAc formation in PDGF-induced VSMC proliferation and osteopontin expression. Increasing flux to the HBP results in increased activity of the enzyme O-GIcNAc transferase (OGT) [226] that attaches GICNAc moieties to protein molecules [220]. OGlcNAcase removes these GlcNAc moieties in a reverse reaction [219]. The global levels of protein O-GlcNAcylated can thus be increased by either, increasing flux into the HBP (increasing OGT activity) or by inhibiting the enzyme O-GIcNAcase. We therefore treated VSMCs with Thiamet-g to determine the effects of increasing protein O-GICNAc formation on VSMC proliferation and OPN gene expression. At non-toxic doses, Thiamet-g caused a concentration dependent increase in protein O-GIcNAc formation (Figure 13A-D). This increase in protein O-GlcNAcylation resulted in inhibition of proliferation induced by PDGF (Figure 14A) and expression of cell cycle proteins PCNA and cyclin D1 (Figures 14B-D).

To verify whether the anti-proliferative effect of Thiamet-g was due to impaired upstream PDGF signaling, we measured the phosphorylation status of Akt and Erk1/2 proteins which are activated upon PDGF stimulation and are known to mediate PDGF mitogenic effects in VSMCs [50]. As shown in Figure 15, Thiamet-g caused no observable difference between PDGF stimulated and unstimulated groups. This further confirmed that increasing protein O-GlcNAc formation inhibits proliferation by inhibiting expression of cell cycle proteins and 
not by interrupting upstream PDGF signaling. We further measured expression of the synthetic cytokine, OPN, in the presence of Thiamet-g. As shown in Figure 16, osteopontin mRNA expression was inhibited in the presence of thiamet-g. This result is consistent with the effect of KA on PDGF-induced VSMC proliferation and OPN expression and suggests that the anti-proliferative effect of Thiamet-g may be mediated in part by inhibition of OPN expression.

Because the expression of cell cycle proteins, PCNA and cyclin D1 were inhibited by thiamet-g, we went further to determine if Thiamet-g had any effect on cell cycle progression. Using propidium iodide staining, we found that in the presence of Thiamet-g, PDGF stimulated cells showed significantly slower progression into the $\mathrm{S}$ and G2/M phases of the cell cycle (Figure 17). 
Figure 11. PDGF-induced glycolytic flux is required for proliferation and osteopontin expression. A-C) Effects of koningic acid on PDGF-induced proliferation. Serum starved VSMCs were treated for 24 with PDGF $(20 \mathrm{ng} / \mathrm{ml})$ in the absence or presence of koningic acid $(0.5 \mu \mathrm{g} / \mathrm{ml})$. Cell proliferation was measured using MTT assay (A) or by counting cell numbers (B). C) LDH assay after koningic acid treatment. Concentration dependent effects of koningic acid on cytotoxicity. ${ }^{*} \mathrm{p}<0.05$ vs control $(0 \mu \mathrm{g} / \mathrm{ml}$ koningic acid). D) Quantitative real time PCR. Osteopontin mRNA expression in the presence of koningic acid \pm PDGF. Serum starved VSMCs were stimulated with PDGF \pm koningic acid for $24 \mathrm{~h}$ and gene and protein abundance measured by real time PCR (D) and immunoblotting (E and F), respectively ( $n=3,{ }^{*} p<0.03$ vs. control). 
Figure 11

A
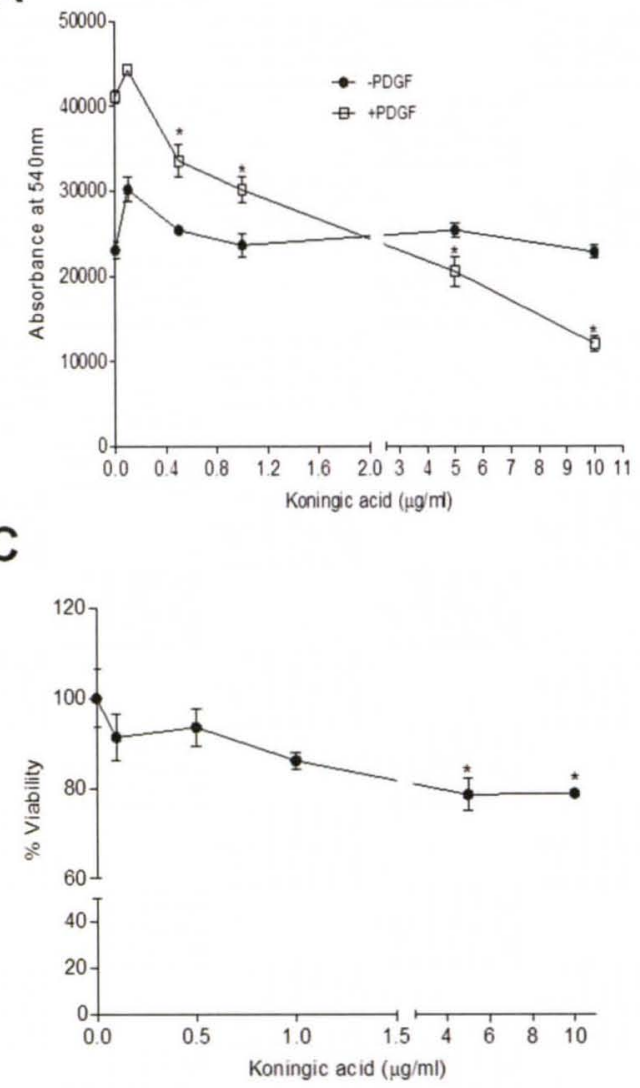

E

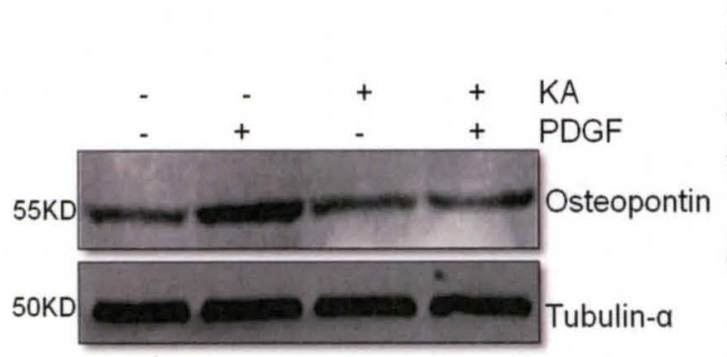

B

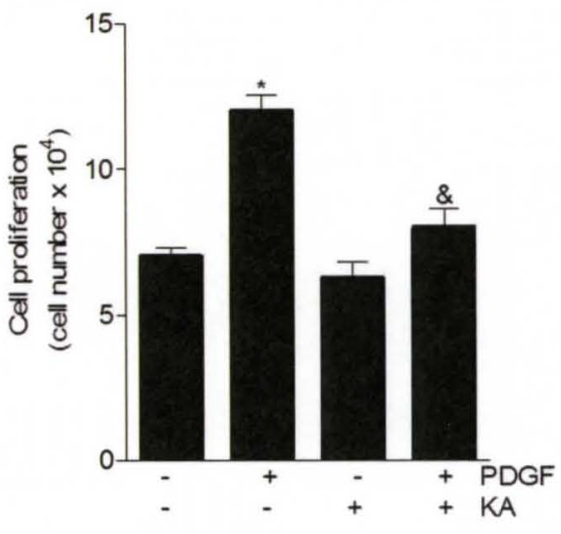

D

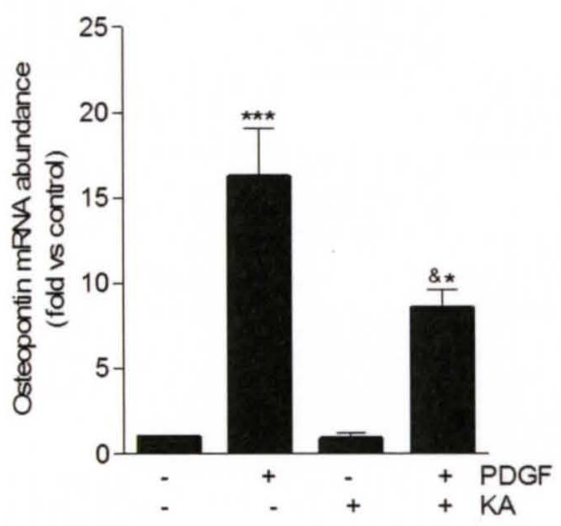

F

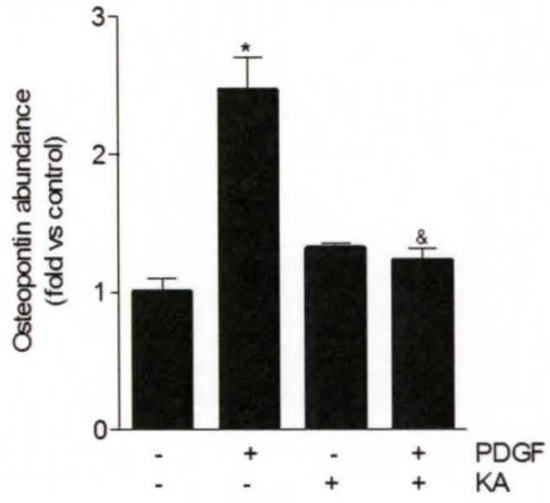


Figure 12. Inhibiting glycolytic flux increases HBP flux. Immunoblot analysis of protein O-GIcNAc formation after koningic acid treatment. A) Increase protein O-GIcNAcylation after koningic acid treatment. B) Quantification of A ( $n=3$, ${ }^{*} p<0.05$ vs. $\left.0 \mu \mathrm{g} / \mathrm{ml}\right)$. 
Figure 12

A

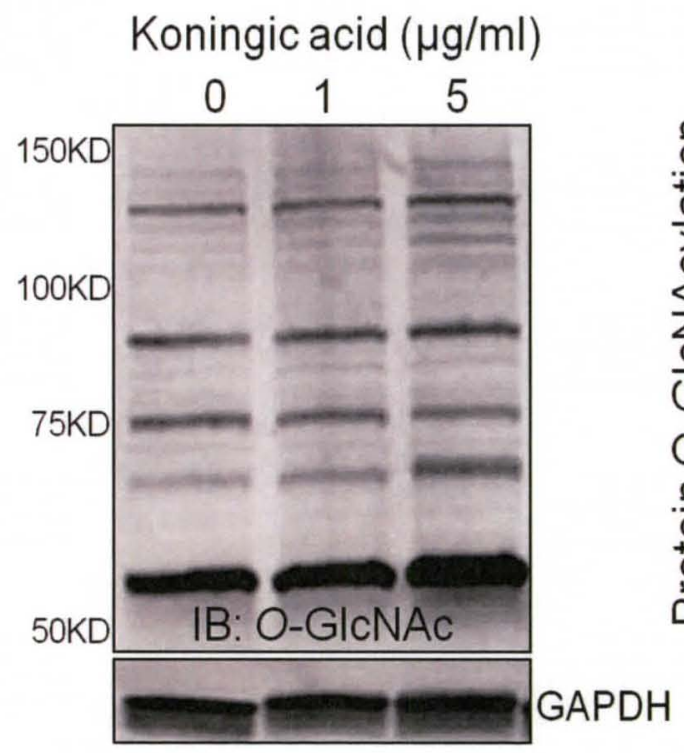

B

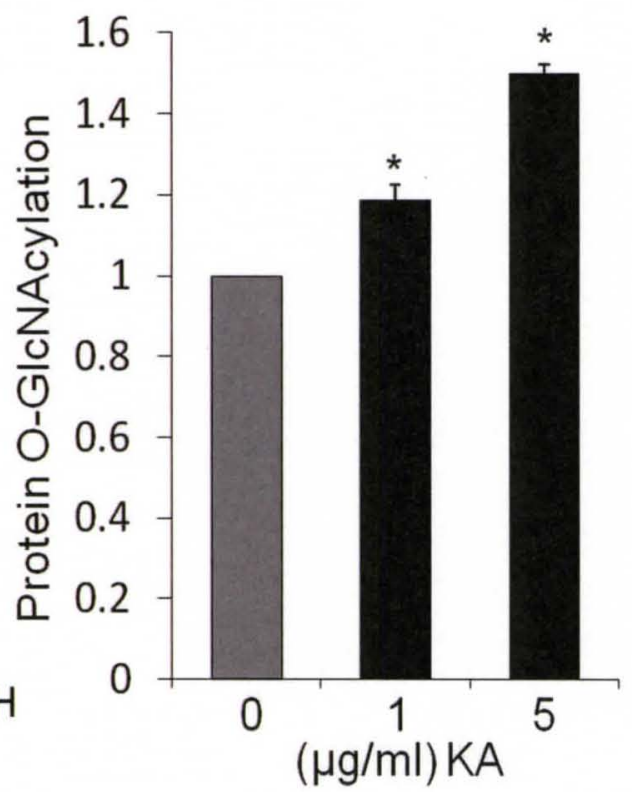




\section{Discussion}

The present study shows a role for glycolysis in PDGF-induced osteopontin expression and PDGF-induced VSMC hyperplasia. This study also demonstrates the importance of protein O-GIcNAc modification in regulating PDGF-induced hyperproliferation. Using the inhibitor of glycolysis, koningic acid, we found that after PDGF treatment, the expression of the proinflammatory cytokine osteopontin was inhibited. Interestingly, inhibiting glucose flux into glycolysis by $\mathrm{KA}$ treatment led to increased protein O-GIcNAc formation. Independently increasing the levels of protein O-GIcNAc formation by inhibiting the enzyme $\mathrm{O}$ GIcNAcase also led to the inhibition of osteopontin expression and expression of cell cycle proteins PCNA and cyclin D1 causing the delay of cell cycle progression. These findings uncover a new role of glycolysis and O-GIcNAC protein modification in regulating the expression of the synthetic cytokine osteopontin and in regulating VSMC proliferation.

Regulation of osteopontin expression is known to be under the influence of different transcription factors. For example, CREB, NFAT, AP-1, USF, SP1, Myc and Oct-1 are known to regulate osteopontin expression after PDGF stimulation and osteopontin expression is necessary for some PDGF-induced changes in the VSMCs $[114,201,227-229]$. Some of these transcription factors are under the control of cellular glucose levels and glucose uptake, and glycolysis is known to increase VSMC hyperplasia induced by PDGF $[198,201]$. However, the role of glycolysis in OPN expression has remained unclear. 
Other pathways of glucose metabolism such as the polyol pathway are known regulators of VSMC hyperplasia. In fact, administering sorbinil, an aldose reductase inhibitor, prevented VSMC hyperplasia in culture and after balloon injury of the carotid artery [230-231] indicating an important role of this pathway in VSMC hyperproliferation. Also, although controversial, the HBP has been implicated in VSMC hyperplasia and neointima [203, 222-223]. Here, we present data that supports an anti-proliferative role of increasing protein O-GIcNAc. Our studies highlight new findings implicating the role of glycolysis and protein $\mathrm{O}$ GIcNAc in OPN expression. We showed that increasing protein O-GIcNAc formation has an inhibitory effect on VSMC hyperplasia and this inhibitory effect could be mediated, in addition to inhibiting cell cycle protein expression, through decreased OPN expression.

The fact that protein O-GIcNAc formation was increased in the presence of KA suggests that glucose metabolism can be regulated and redirected into a different pathway upon perturbation in one pathway. The increased protein $\mathrm{O}$ GIcNAc formed after koningic acid treatment suggests that the antiproliferative effects of inhibiting glycolysis (i.e., by KA treatment) could be mediated in part by protein O-GIcNAc formation. These are important observations although the molecular mechanism(s) leading to these observed effects have not been rigorously tested. However, it is possible that the changes in glucose metabolism caused by PDGF regulates glucose-controlled transcription factors such as upstream stimulatory factor (USF) leading to inhibition of osteopontin expression and VSMC proliferation. We noticed that progression of the cell cycle through 
the S-phase was significantly delayed in the presence of thiamet-g thereby suggesting that mechanisms regulating S-phase transition could have been inhibited. The inhibitors, koningic acid and thiamet-g did not alter PDGF-induced autophagy (observed in chapter II) or PDGF-induced degradation of contractile proteins thereby suggesting that the process of cellular degradation during phenotype switching might simultaneously be occurring via a pathway independent of osteopontin expression and proliferation.

In summary, the study presented in this section identifies possible roles of glucose metabolic pathways in regulating VSMC proliferation and osteopontin expression. This study highlights the importance of glucose metabolism in attaining the fully synthetic phenotype characterized by, in addition to decreased contractile protein expression, increased osteopontin expression and increased proliferation. Our study suggests possible therapies that could be developed and used (in drug eluting stents) to curb VSMC hyperproliferation. Future studies are needed to delineate the complete mechanism by which these inhibitors produce their effects. 
Figure 13. Thiamet-g increases protein O-GIcNAcylation in VSMCs. Immunoblot analysis of protein O-GIcNAc formation after thiamet-g treatment. A) Serum starved VSMCs were treated with Thiamet-g at the indicated concentrations. B) Densitometric quantification of $A\left(n=3,{ }^{*} p<0.03\right.$ vs $\left.0 \mu M\right)$. C and D) Cytotoxicity due to Thiamet-g treatment. Cell toxicity was measured using the MTT assay and the LDH assay ( ${ }^{*} p<0.01$ vs control). Thapsigargin was used as a positive control to induce cell death. 
Figure 13

A

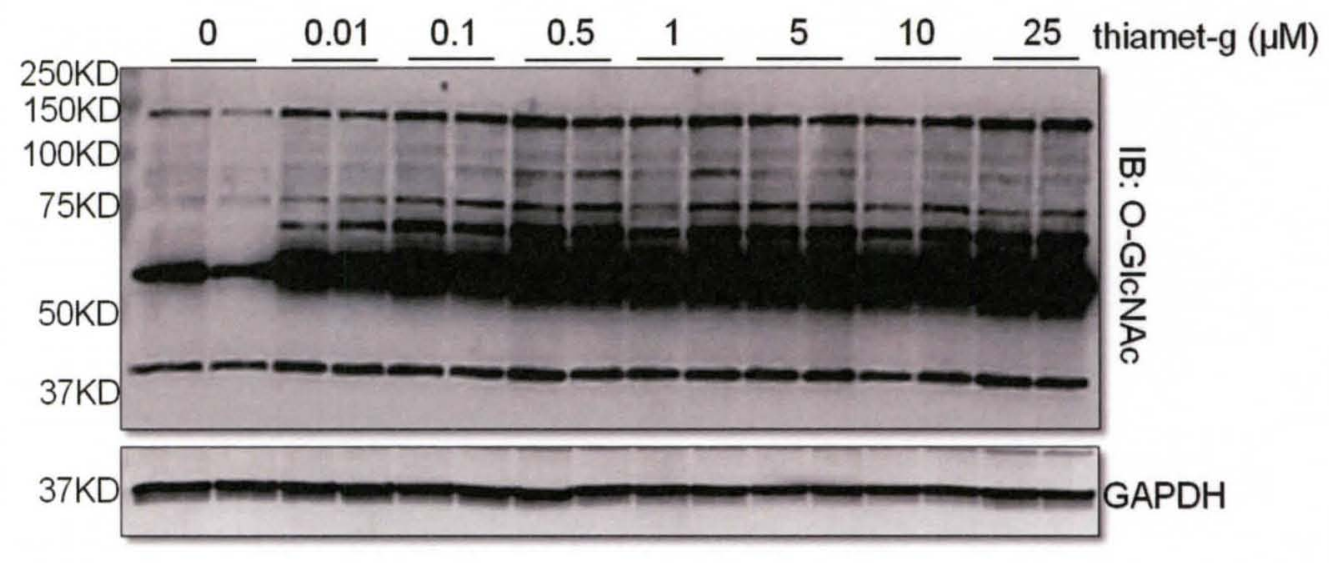

B

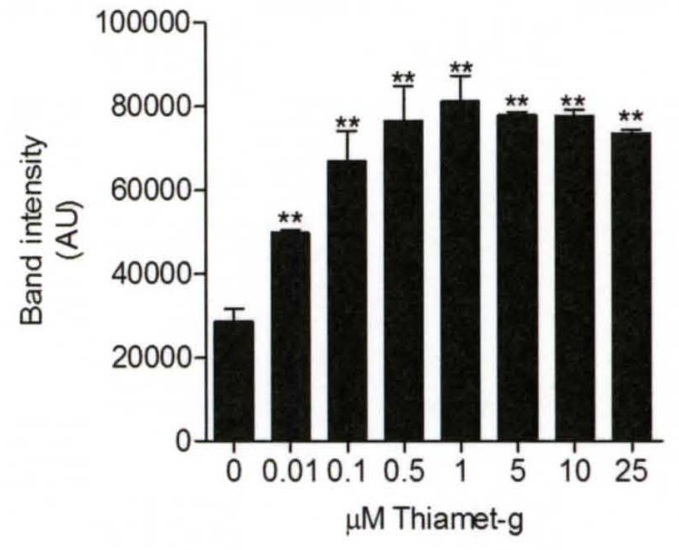

C

D
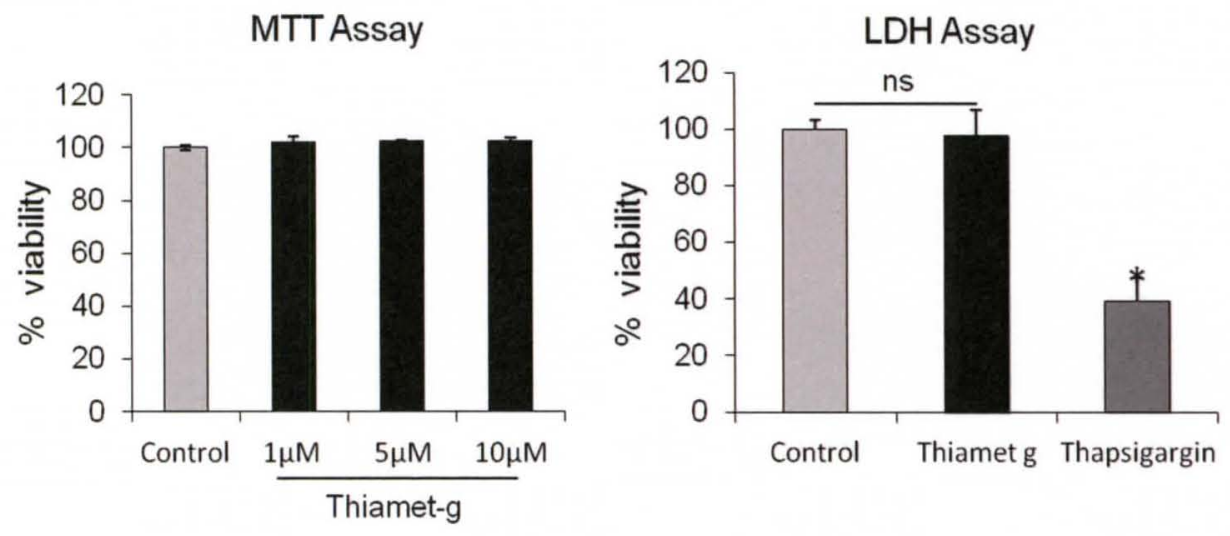
Figure 14. Increased protein O-GIcNAcylation inhibits PDGF-induced VSMC proliferation. Cell proliferation assay. (A) Serum starved VSMCs were treated with PDGF $(20 \mathrm{ng} / \mathrm{ml}) \pm$ Thiamet-g and proliferation measured by counting cell numbers ( $n=6,{ }^{*} p<0.03$ vs. PDGF). (B) Expression of cell cycle proteins. Immunoblot analysis of PCNA and cyclin D1 abundance after PDGF \pm treatment. (C and $D)$ quantification of $B\left(n=3,{ }^{*} p<0.05\right.$ vs control). 
Figure 14

A
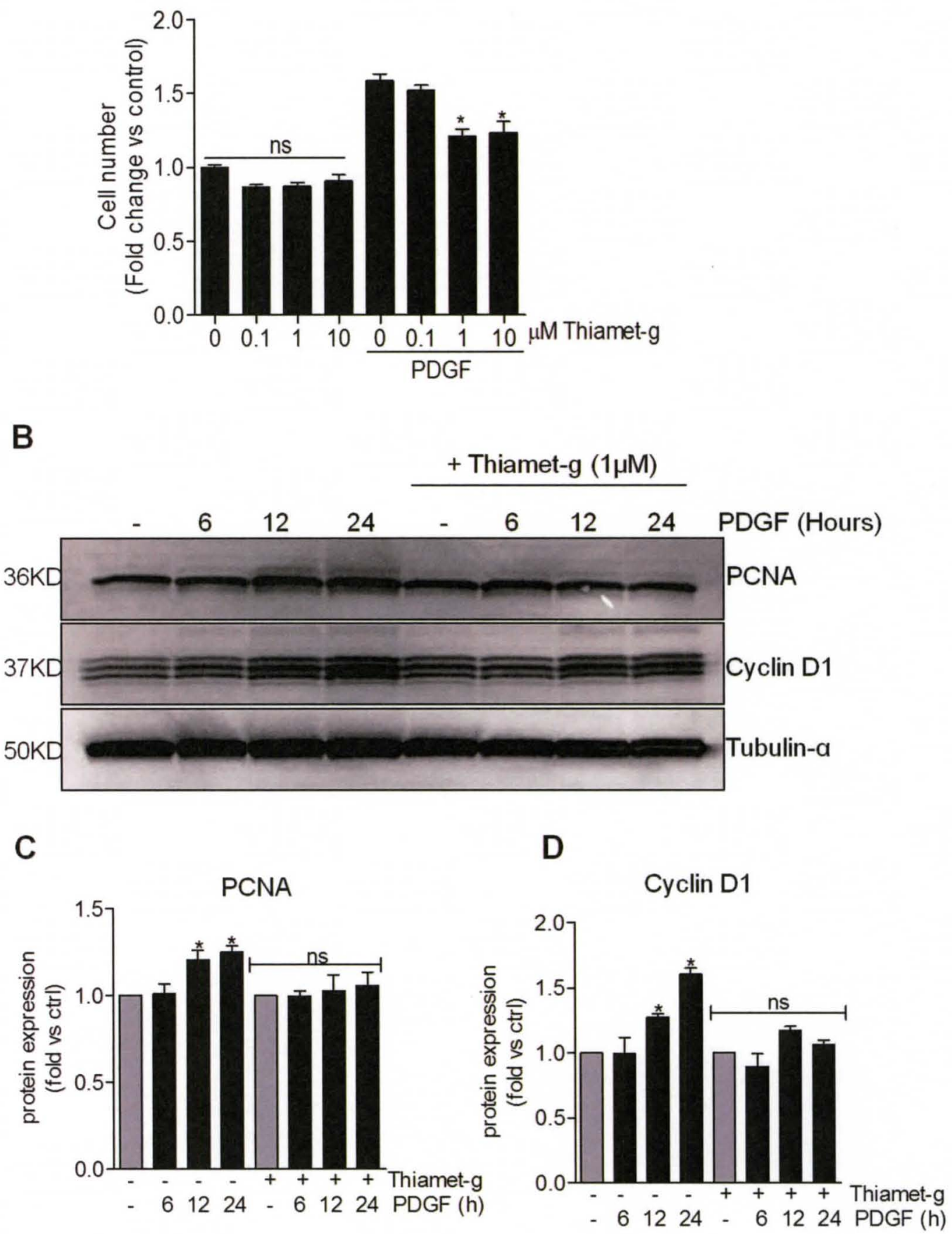
Figure 15. Thiamet-g does not inhibit PDGF signaling. (A) Immunoblot analysis of Akt and Erk1/2 phosphorylation after PDGF \pm Thiamet-g treatment. (B and $C$ ) quantification of $A$ ( $n=3$ per group). 
Figure 15

A

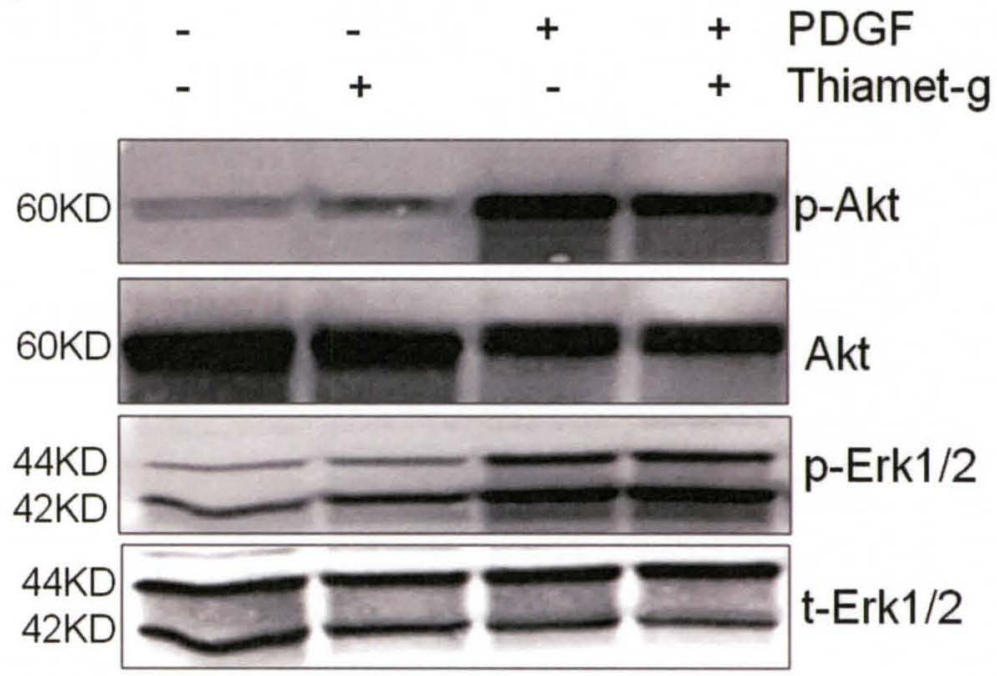

B

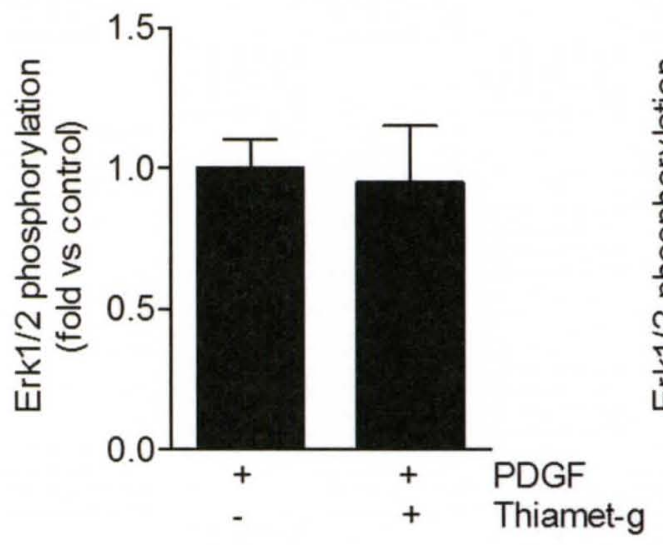

C

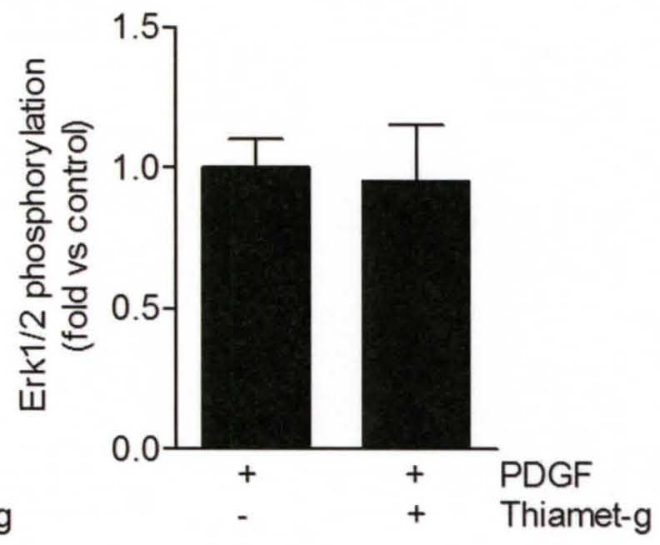


Figure 16. Increased protein O-GIcNAcylation inhibits PDGF-induced osteopontin expression. Quantitative real time PCR. Serum starved VSMCs were treated for $24 \mathrm{~h}$ with PDGF $(20 \mathrm{ng} / \mathrm{ml}) \pm$ Thiamet-g $\left(n=3,{ }^{*} \mathrm{p}<0.01\right.$ vs control and ${ }^{*} p<0.01$ vs PDGF). 
Figure 16

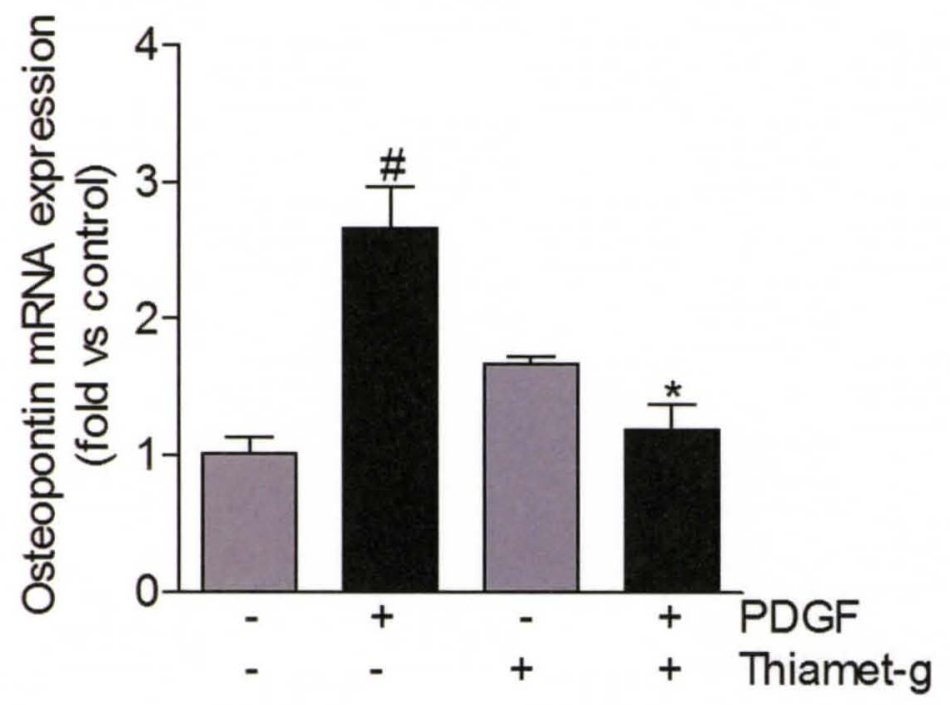


Figure 17. Thiamet-g causes delayed cell cycle progression. Cell cycle analysis with propidium iodide. VSMCs serum starved for $24 \mathrm{~h}$ and then stimulated with PDGF or PDGF + Thiamet-g for the indicated time points. Cell cycle progression was monitored using a C6 Accuri flow cytometer $(n=3$, ${ }^{*} p<0.001$ for PDGF vs PDGF/Thiamet-g). 
Figure 17
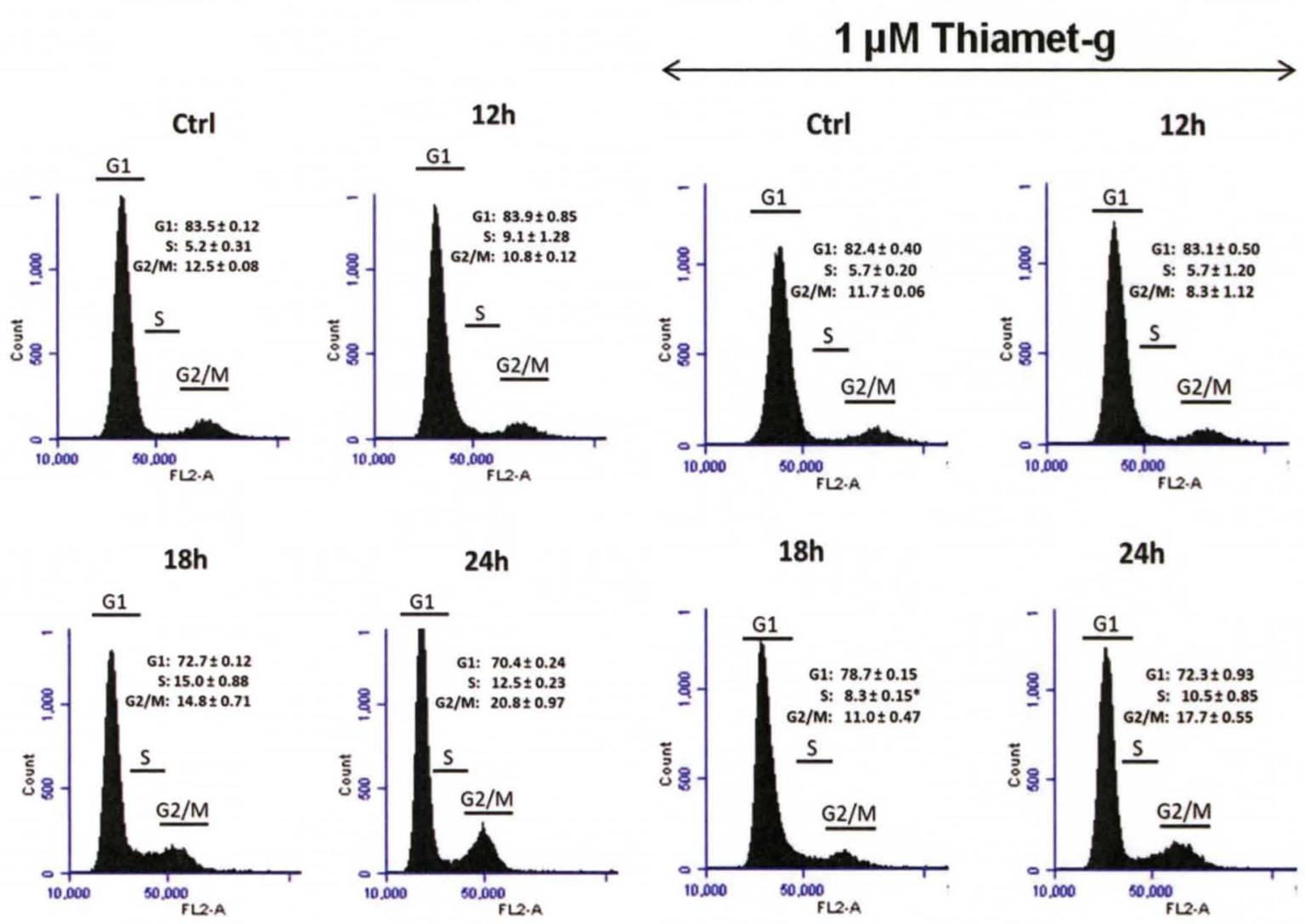


\section{CHAPTER IV}

\section{MITOCHONDRIA FISSION REGULATES METABOLISM AND VSMC PROLIFERATION}

\section{Introduction}

Rapidly proliferating cells require an increase in energy production and elevated formation of metabolic intermediates. These demands can be achieved by increasing the metabolism of major substrates such as glucose, glutamine or fatty acids. In synthetic VSMCs which are not only rapidly dividing, but are migratory and secreting ECM, the requirements for cellular energy are even higher. To meet with these demands, synthetic VSMCs could increase their overall metabolic flux or switch to oxidizing metabolic substrates that provide more energy. Thus, metabolizing more efficient substrates, such as fatty acids could be an efficient means of satisfying cellular needs during excessive proliferation and migration.

Mitochondria are the cellular hubs of metabolism and have the capacity of both changing their substrate preference as well as their morphology. Previously, mitochondria were regarded as static organelles with the principal function of producing ATP needed for cellular respiration. However, with the discovery of different proteins that regulate mitochondrial morphology, these organelles are 
now known to be dynamic and capable of changing their morphology to regulate different cellular processes, such as calcium handling [232] and apoptosis [233]. During cell proliferation, mitochondria form giant networks which are known to be associated with increased ATP formation [234]. This network formation typically occurs during the $\mathrm{G} 1$ to $S$ transition of cell cycle in which the cellular glycolytic rates are low. Thus, mitochondria network formation maximizes energy production during these times of increased synthetic activity.

A number of mitochondria related proteins are now known to regulate mitochondria morphology. For example dynamin related protein 1 (DRP1) and human mitochondria fission protein 1 (hFis1) promote mitochondria fission [235236]. Conversely, mitofusins 1 and 2 (Mfn1 and 2) and optic atrophy 1 (Opa1) promote mitochondrial fusion. Changes in mitochondrial morphology (mitochondrial fragmentation) occurs in apoptotic cell death [237-238]. Recent studies have shown that mitochondrial morphology is instrumental in the process of mitophagy whereby long tubulated mitochondria are spared from incorporation into the autophagosome [239]. Mitochondrial morphology in diseased conditions such as ischemia reperfusion injury, diabetes, cytosolic calcium overload, heart failure and dilated cardiomyopathy has also been studied [240-243]. However, the role of mitochondria in the VSMC during phenotype conversion has not been given great attention with only a few recent studies suggesting a role of Mfn2, Drp1 and mitochondria morphology in VSMC hyperproliferation under diseased conditions [244-245]. 
In this study, we examined the effects of PDGF on mitochondrial morphology and metabolism. We found that synthetic VSMCs utilize increase fatty acid oxidation (FAO) in response to PDGF which was preceded by mitochondria fragmentation. Mitochondria fragmentation induced by PDGF was accompanied by decreased expression of the pro mitochondria fusion gene mitofusin 2 thereby suggesting a mitofusin 2-mediated mechanism. Mitochondria fragmentation was necessary for increased FAO and increased proliferation because mdivi-1, an inhibitor of mitochondria fragmentation, prevented PDGF-induced FAO and proliferation. This increase in FAO was not due to an increase in mitochondria content because the total mitochondria abundance did not change in the synthetic cells. Collectively, these data demonstrate that substrate switching to FAO is important in generating energy needed for the synthetic VSMC and identifies mitochondria fragmentation as an important process critical for VSMC proliferation after PDGF stimulation. 


\section{Experimental procedures}

\section{Materials}

Reagents: Antibodies against VDAC, COX IV and ATP synthase $\beta$ were obtained from Novus Biologicals (Littleton, CO, USA). The Mito ID red dye and Hoechst stain were obtained from Enzo life sciences (Farmingdale, NY, USA). Etomoxir, FCCP, antamycin A and rotenone were obtained from Sigma Aldrich (St. Louis, MO, USA). Rabbit polyclonal antibody against rat Mfn2 was obtained from Abcam. Recombinant FGF-1 was obtained from eBiosciences (San Diego, CA, USA). Antibodies against $\alpha$-smooth muscle cell-actin (a-SMA), calponin and a-tubulin were purchased from Sigma-Aldrich (St. Louis, MO, USA). Rabbit polyclonal antibody against LC3 was purchased from Cell Signaling Technologies (Danvers, MA, USA). Propidium iodide was purchased from eBiosciences (San Diego, CA, USA).

Cell culture: Rat aortic smooth muscle cells (RASMCs) were isolated from the aortas of 6 weeks old male Sprague-Dawley rats and grown in DMEM (Dulbecco's modified Eagle's medium; Life Technologies-Invitrogen) supplemented with $10 \% \quad(\mathrm{v} / \mathrm{v})$ FBS (foetal bovine serum) and $0.1 \%$ streptomycin/penicillin. Cells used in experiments were from between passages 2-7 maintained in a humidified atmosphere of air and $5 \% \mathrm{CO}_{2}$ at $37{ }^{\circ} \mathrm{C}$. At about $70 \%$ confluency, RASMCs were serum starved in DMEM containing $0.1 \%$ FBS for 24 hours. After desired treatments, cells were rinsed twice with phosphate buffered saline and then lysed in a protein lysis buffer containing 25 mM HEPES, 
$1 \mathrm{mM}$ EDTA, $1 \mathrm{mM}$ EGTA, $0.1 \%$ SDS, 1\% NP-40, 1x protease and phosphatase inhibitors. Lowry's assay was used for measuring protein concentration of crude cell extracts.

Western blotting: For Western blotting, between $0.5-25 \mu \mathrm{g}$ of crude cell protein was applied to each lane of a $10.5-14 \%$ Bis-Tris gel and electroblotted onto a PVDF membrane. The membrane was then incubated overnight at $4^{\circ} \mathrm{C}$ using appropriate dilutions of primary antibodies. The PVDF membranes were incubated at room temperature with horseradish peroxidase-conjugated secondary antibodies. Immunoreactive bands were detected with enhanced chemiluminescent detection reagent (GE Healthcare).

Confocal Imaging: Cells plated on glass-bottom dishes were serum starved for 24h in $0.1 \%$ FBS DMEM. Cells were then stimulated with PDGF and desired cotreatments for the desired times. For visualization, mitochondria were stained using the mito ID kit from Enzo life sciences (Farmingdale, NY, USA) following the specified protocol. Mito ID and Hoechst stains were diluted in phenol red-free DMEM supplemented with $25 \mathrm{mmol} / \mathrm{L}$ HEPES, $\mathrm{pH} \mathrm{7.4)} \mathrm{and} \mathrm{then} \mathrm{added} \mathrm{to} \mathrm{cells}$ for 10 mins. For imaging, cells were illuminated with desired laser and images acquired using a $60 x$ objective lens covered with a thin layer of oil.

Measurement of cellular energetics using the XF24 extracellular flux analyser: Cellular energetics was measured in intact VSMCs using a Seahorse Bioscience XF24 extracellular flux analyzer. Fifty thousand cells were seeded in 12-wells of a 24-well XF plate (Control group), while twenty five thousand cells were seeded in the remaining 12 wells (PDGF treated group). Cells were then 
serum starved in $0.1 \%$ FBS DMEM for $24 \mathrm{~h}$ and then stimulated with $20 \mathrm{ng} / \mathrm{ml}$ PDGF in the same media for $48 \mathrm{~h}$. After PDGF stimulation, media was replaced with DMEM containing $100 \mu \mathrm{M}$ BSA-palmitate, $1 \mathrm{mM}$ pyruvate, $5 \mathrm{mM}$-glucose, and $100 \mu \mathrm{M}$ L-carnitine. After equilibration in the media, the cells were exposed to etomoxir and OCR was measured. Data obtained were normalized to the amount of protein present in the well of the microplate. For normalization, cells were lysed in the 24-well XF plates using $20 \mu \mathrm{l} /$ well of protein lysis buffer. Protein concentration was then measured using the Lowry's method.

Cell proliferation assays and measurement of cell viability: Vascular smooth muscle cells were serum starved for $24 \mathrm{~h}$ in order to synchronize cells in the G0 phase. After serum starvation, cells were treated for the required duration as indicated. After treatment, cells were rinse twice with PBS and then trypsinized with a $0.25 \%$ trypsin solution for about 3 minutes at $37^{\circ} \mathrm{C}$. Cells were then resuspended in DMEM containing 10\% FBS and cell numbers were determined using a C6 Accuri flow cytometer. For viability assays, after desired treatment, cells were incubated in a solution of $0.2 \mathrm{mg} / \mathrm{ml} \mathrm{MTT}$ reagent for $2-4 \mathrm{~h}$ at $37^{\circ} \mathrm{C}$ in an incubator containing $5 \% \mathrm{CO}_{2}$. After incubation, MTT reagent was then aspirated and the purple crystals at the bottom of the plate dissolved in DMSO. Absorbance at $540 \mathrm{~nm}$ was then measured using a microplate reader.

Cell cycle studies with propidium iodide: VSMCs were harvested after 3 min trypsin incubation, washed with phosphate buffered saline (PBS) and cold $70 \%$ ethanol added to the cells. VSMCs were then kept at $4^{\circ} \mathrm{C}$ for 1 hour to fix cells. 
After fixing, cells were sedimented at $500 \times \mathrm{g}$ for 5 mins and the supernatant discarded. Cells were then washed again with PBS and $300 \mu$ RNase buffer containing $5 \mu$ propidium iodide added after all residual PBS has been discarded. Cells were then mixed and incubated at room temperature for $45 \mathrm{~min}$ after which they were analyzed using a C6 Accuri flow cytometer.

Statistical analysis: Data are mean \pm SEM. Experimental groups were compared using one-way ANOVA, followed by Bonferroni post-tests. Unpaired Student's $t$ test was used for direct comparisons. A p-value $<0.05$ was considered significant.

\section{Results}

\section{Synthetic VSMCs show increased fatty acid oxidation and mitochondria}

fragmentation: To examine energetic changes that occur in synthetic VSMCs, we measured fatty acid oxidation (FAO) after PDGF stimulation. We serum starved VSMCs and then converted one group to the synthetic phenotype by PDGF treatment for $48 \mathrm{~h}$, while the other group was left untreated. Oxygen consumption was then measured in media containing L-carnitine and only BSApalmitate and glucose as available substrates. To interrogate the contributions of FAO and glucose oxidation to the overall oxidative phosphorylation, we added etomoxir into the media and then monitored oxygen consumption rates (OCRs). Etomoxir is an inhibitor of carnitine palmitoyl transferase 1, an enzyme needed for conjugating and translocating long chain fatty acids from the cytoplasm to the 
mitochondria where they are committed to $\beta$-oxidation [246]. Addition of etomoxir should therefore cause a drop in OCR and this drop in OCR will be due to inhibition of $\beta$-oxidation or FAO (see scheme 5). As shown in Figure 18, etomoxir injection decreased in OCR to a greater extent in PDGF treated cells compared with control cells, thereby indicating an increase in FAO in synthetic cells. This increase in FAO could not be due to differences in the amount of cells nor changes in mitochondria abundance because the results were normalized to protein expression per well and because a Western blot analysis of mitochondria content showed no differences between the synthetic and contractile phenotypes (Figure 19). 


\section{Scheme 5}

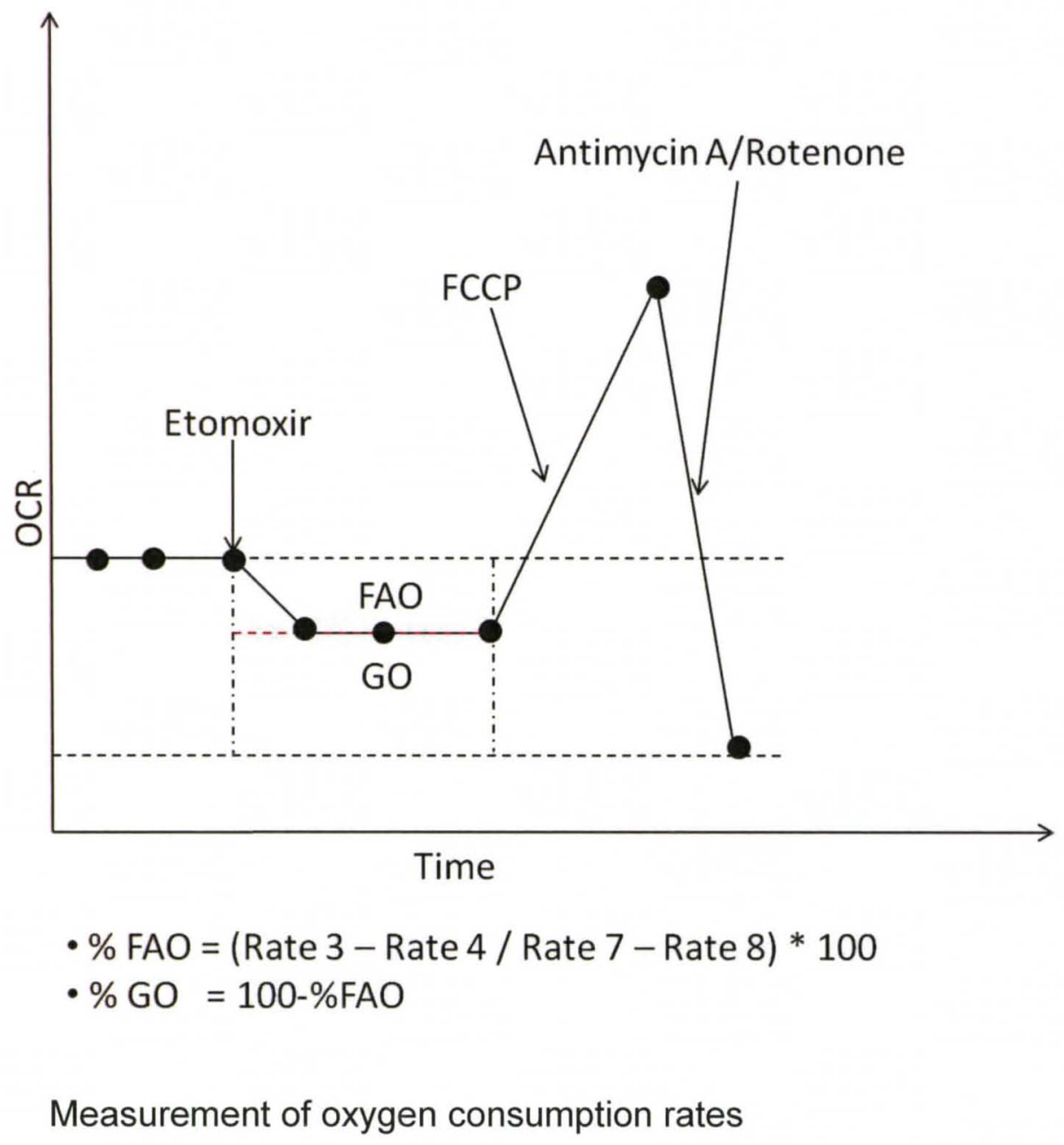


Figure 18. PDGF treatment increases fatty acid oxidation in VSMCs. Oxygen consumption measurements in contractile and synthetic VSMCs A) Representative extracellular flux measurements. VSMCs were serum starved for $24 \mathrm{~h}$ and then left untreated or converted to the synthetic phenotype by PDGF stimulation for $48 \mathrm{~h}$ (OCR drop after etomoxir injection is fatty acid linked). OCRs were normalized to total proteins. B and C) Quantification of A. (Total oxidative phosphorylation activity $=($ point $7-$ point 8$)$. Percent oxygen consumption due to $\mathrm{FAO}=($ point $3-$ point 4$) /(\text { point } 7-\text { point } 8)^{\star} 100$. Percent due to $\mathrm{GO}=(100-$ $\% F A O)$, see scheme $5 .\left(n=3,{ }^{*} p<0.05\right.$ vs control i.e. contractile cells). 
Figure 18

A

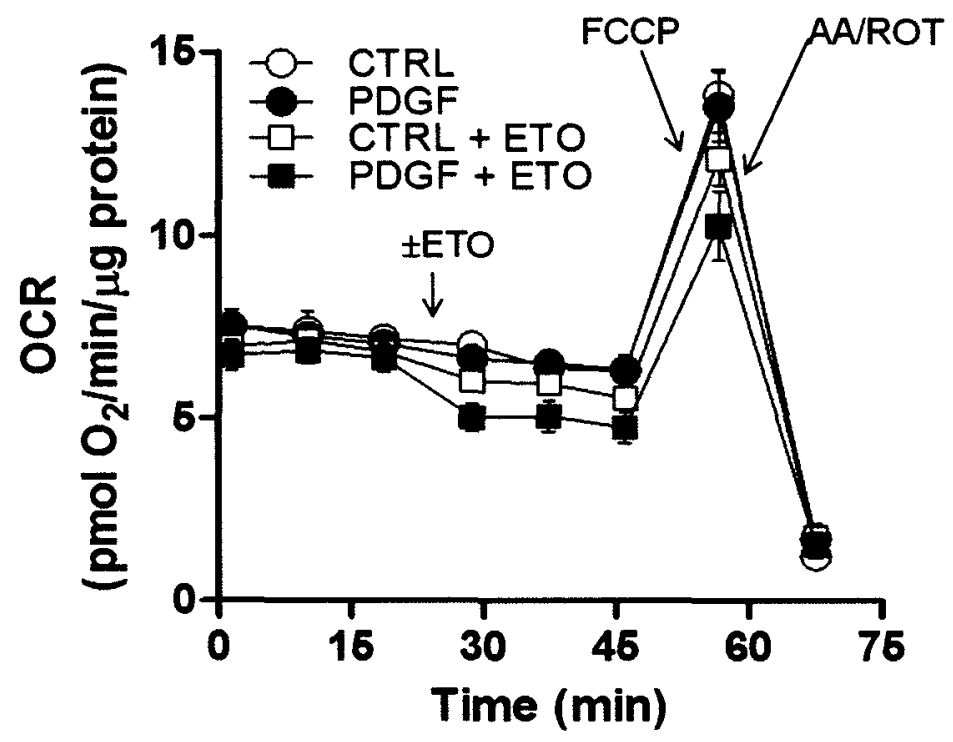

B

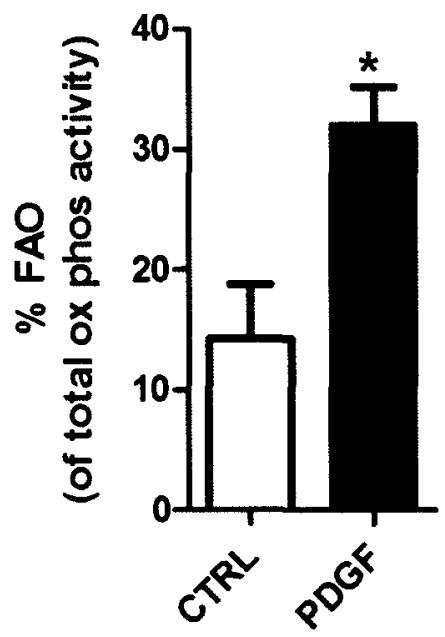

C

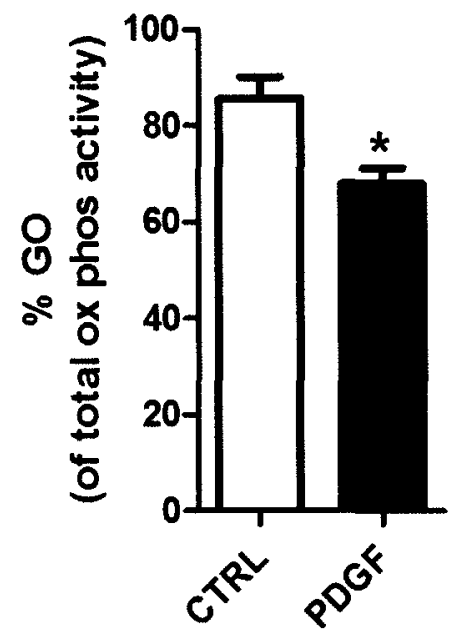


Figure 19. Increased FAO in synthetic VSMCs is not due to increased mitochondria abundance. Total mitochondria abundance was not changed upon conversion to the synthetic phenotype. A) Representative Western blot. VSMCs were starved for $24 \mathrm{~h}$ and then treated without or with PDGF for $48 \mathrm{~h}$. BE) Quantification of $A$, ( $n=3$ independent experiments). 
Figure 19

A \begin{tabular}{lllllll}
- & 0.5 & 3 & 6 & 12 & 24 & 48 \\
\hline
\end{tabular}

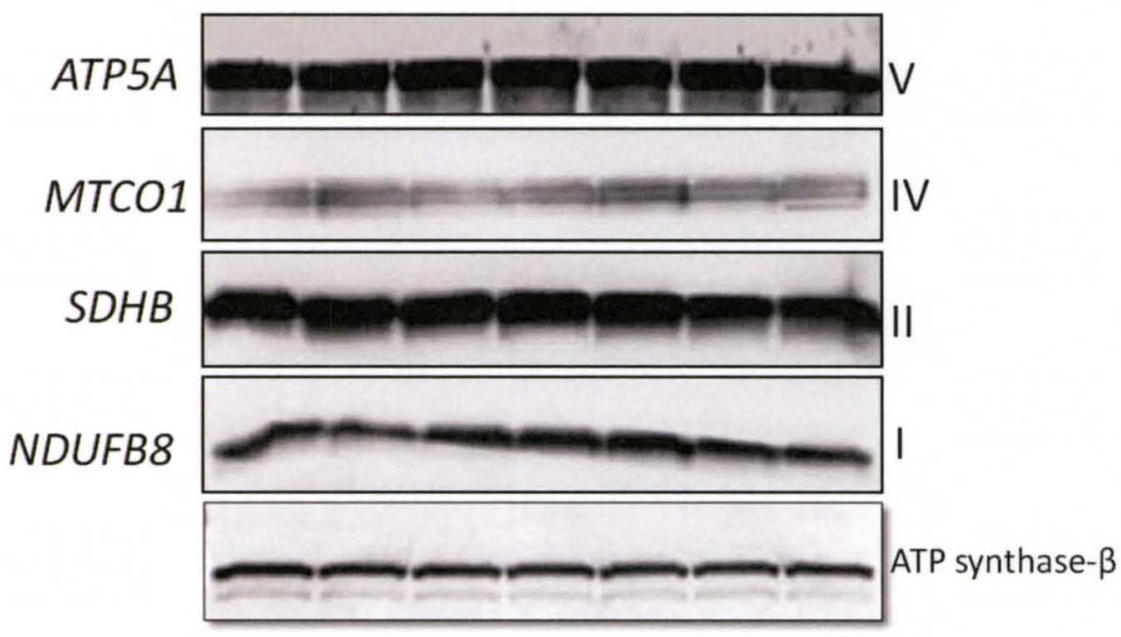

B

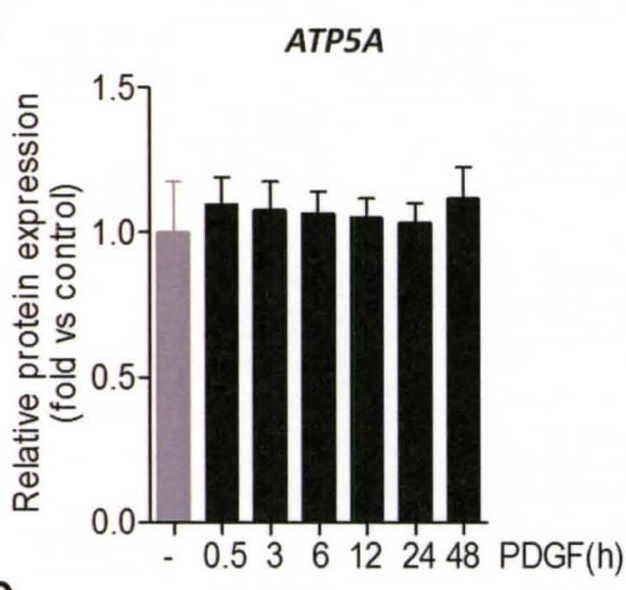

D
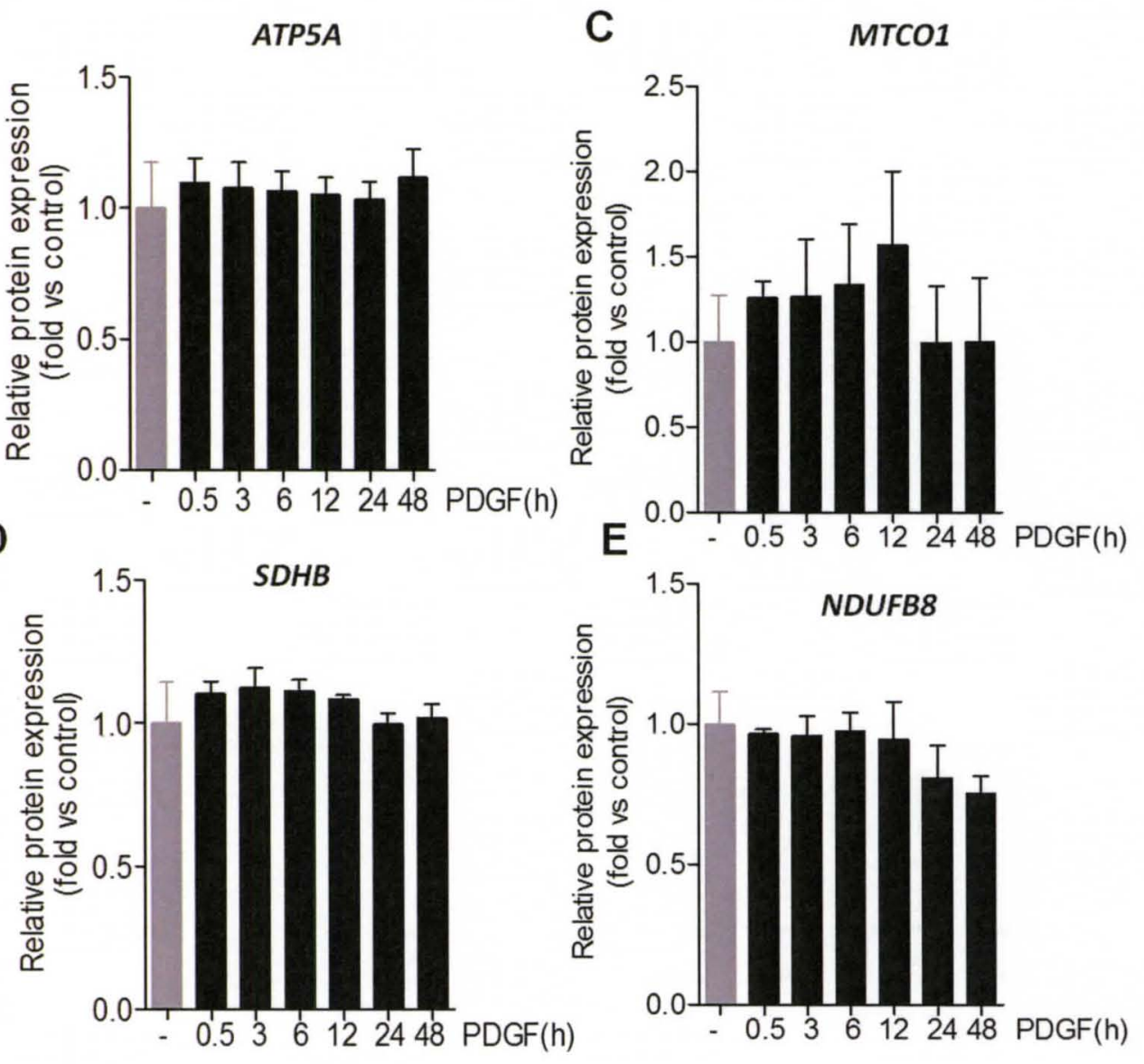
It was recently reported that changes in mitochondrial morphology and network formation play important roles in energy production and proliferation of VSMCs [247-248]. We therefore went further to determine whether the observed change in substrate utilization was accompanied with changes in mitochondria morphology. As shown in Figure 20A, synthetic VSMCs had mainly small rounded or fragmented mitochondria (globular) as opposed to contractile cells whose mitochondria were mainly filamentous and network forming. The expression of mitofusin 2, a pro-mitochondria fusion gene, was down regulated in synthetic cells thereby suggesting a mitofusin 2-dependent mechanism of mitochondrial fragmentation (Figure $20 \mathrm{C}$ and D). Interestingly, this change in mitochondria shape and substrate utilization was specific to cells stimulated with PDGF because FGF-1 neither increased FAO nor did it cause mitochondrial fragmentation (Figure 21).

Mitochondria fragmentation causes increased FAO in synthetic VSMCs: To determine whether changes in mitochondria morphology influenced substrate usage in synthetic cells, we stimulated VSMCs with PDGF in the presence of the mitochondria fission inhibitor (DRP1 inhibitor), mdivi1 [249] and measured changes in OCR after etomoxir injection. As shown in Figures $22 \mathrm{~A}$ and $\mathrm{B}$, mdivi1 inhibited the fragmentation caused by a switch to the synthetic phenotype. This inhibition also prevented the switch to FAO observed upon conversion to the synthetic phenotype (Figures $22 \mathrm{C}$ and D). The inhibition of mitochondria fragmentation caused a complete blockage of PDGF-induced proliferation by 
decreasing the expression of cell cycle proteins cyclin D1 and PCNA and stalling cells in the s-phase of the cell cycle (Figure 23). 
Figure 20. PDGF induces mitochondria fragmentation in VSMCs. Mitochondria morphology in contractile and synthetic VSMCs. A) Representative live cell confocal images of untreated or 48 hour PDGF treated cells. B) Mitochondrial morphologies. Mitochondria lengths were measured using the NIS Elements software and scored as follow: Mitochondrial morphology was scored as follows: Globular, mainly small and round $(<2 \mu \mathrm{m}$ in diameter); intermediate, between 2-4 $\mu \mathrm{m}$ in length; and filamentous, long and higher interconnectivity (> 4 $\mu \mathrm{m}$ in length). The percentage of cells with indicated mitochondrial morphologies was determined as a percentage of the total number of stained cells counted ( $\geq 150$ cells per experiment), $n=4$ independent experiments, blue $=$ hoechst, red $=$ mito ID red. (Scale bar: $10 \mu \mathrm{m}$ ). C) PDGF-induced mitochondria fragmentation is associated with loss of mitofuscin 2. Representative Western blot analysis of mitofusin 2 expression after PDGF time course stimulation. D) Quantification of $C,\left(n=3\right.$ independent experiments, ${ }^{*} p<0.05$ vs ctrl). 
Figure 20

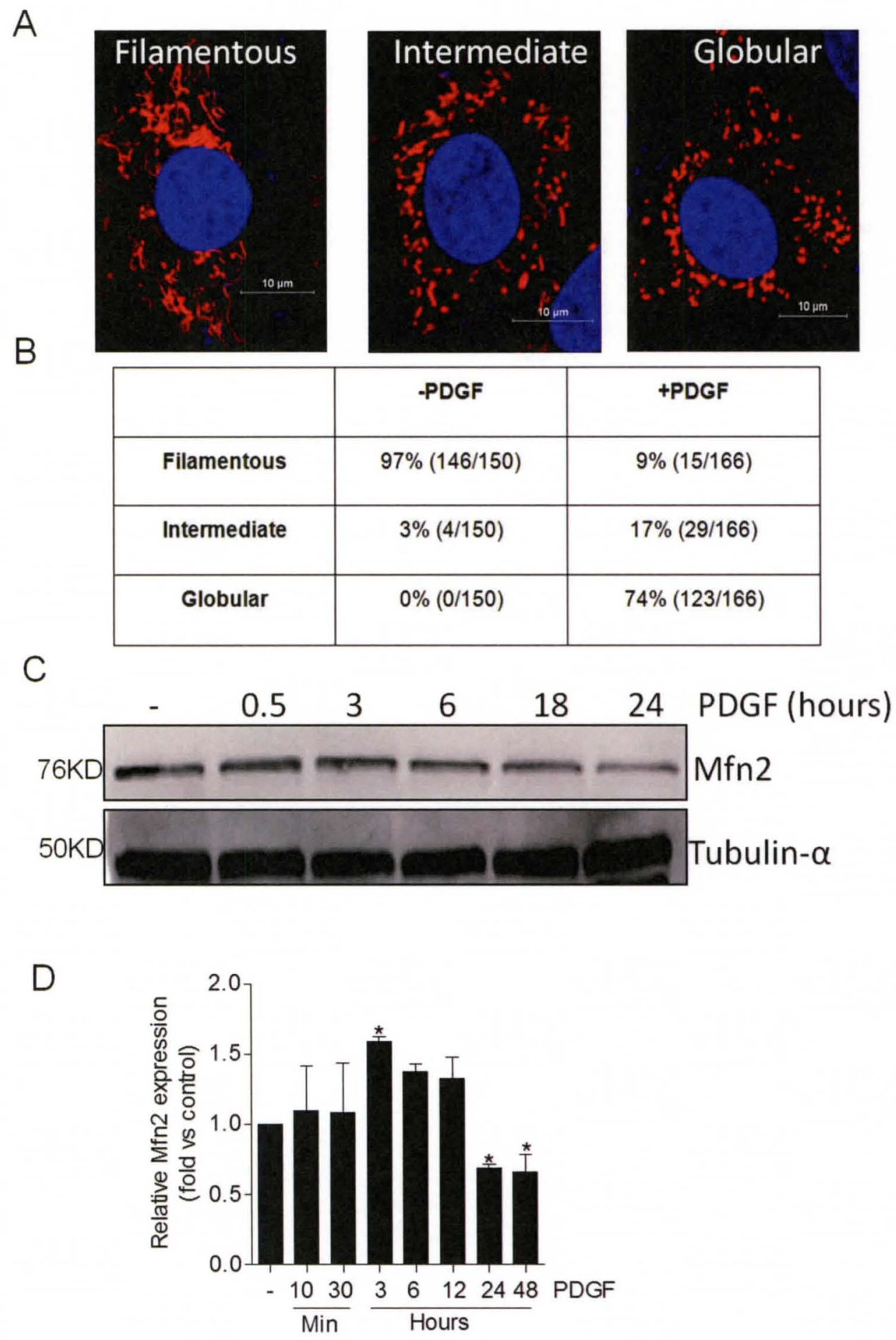


Figure 21. FGF-1 does not induce mitochondria fragmentation/fatty acid oxidation. Mitochondria morphology and FAO in VSMCs exposed to FGF-1. A) Representative extracellular flux measurements. VSMCs were serum starved for 24h and then stimulated with FGF-1 for $48 \mathrm{~h}$. B and C) Quantification of A. Total oxidative phosphorylation activity $=($ point $7-$ point 8$) . \%$ oxygen consumption due to $\mathrm{FAO}=($ point $3-$ point 4$) /($ point $7-$ point 8$) * 100 . \%$ due to $\mathrm{GO}=(100-$ \%FAO). D) Mitochondria morphology after FGF exposure. 
Figure 21

A

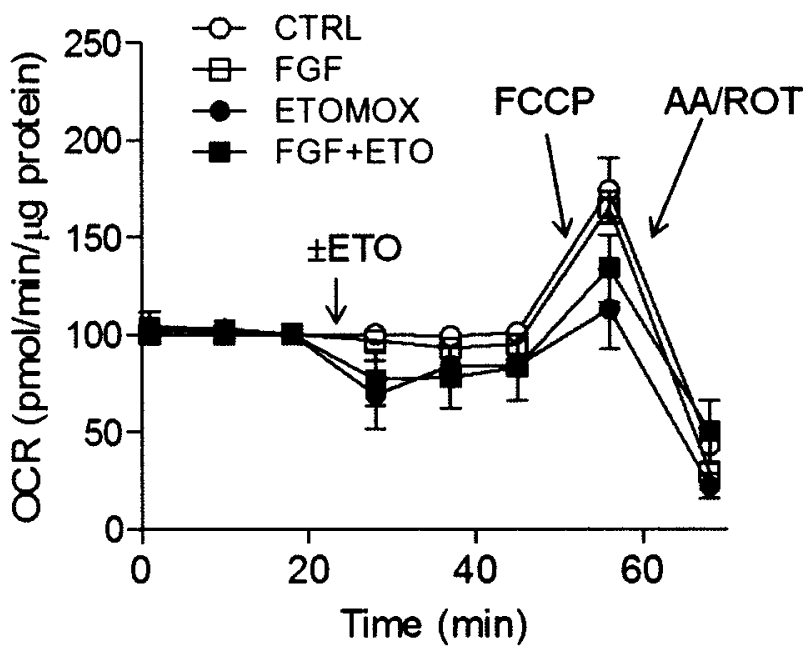

B

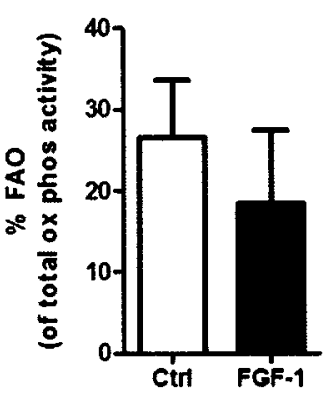

C

D

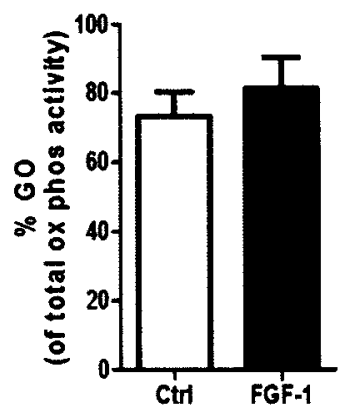

\begin{tabular}{|c|c|c|}
\hline & FGF-1 & +FGF-1 \\
\hline Filamentous & $96 \pm 1 \%(146 / 447)$ & $70 \pm 10 \%(132 / 189)$ \\
\hline Intermediate & $3 \pm 1 \%(4 / 447)$ & $27 \pm 9 \%(51 / 189)$ \\
\hline Globular & $1 \pm 0.5 \%(0 / 447)$ & $3 \pm 1 \%(6 / 189)$ \\
\hline
\end{tabular}


To verify if mitochondrial fragmentation acts upstream of $F A O$, we stimulated cells with PDGF in the presence of etomoxir and then measured changes in mitochondrial morphology. As shown in Figure 24, etomoxir inhibited PDGFinduced proliferation but had no effect on mitochondria fragmentation, suggesting that mitochondria fragmentation precedes increases in FAO and proliferation. Our results suggest that VSMCs in the synthetic phenotype increase FAO and this increase in FAO is regulated by mitochondria morphology because only the globular (fragmented) mitochondria phenotype was associated with increased FAO.

PDGF-induced contractile protein degradation during phenotype switching is NOT dependent on mitochondria fragmentation and FAO: We previously determined that conversion to the synthetic phenotype required induction of autophagy, which is needed for the degradation of contractile proteins such as calponin and $\alpha-S M$ actin (Figure 5). Since inhibition of mitochondria fragmentation inhibited PDGF-induced proliferation and because increased proliferation is associated with the synthetic phenotype, we wanted to verify if mitochondrial fragmentation and increased FAO were required for PDGF-induced contractile protein degradation leading to the emergence of the synthetic phenotype. As shown in Figure 25, inhibition of PDGF-induced fragmentation did not affect degradation of contractile proteins suggesting that contractile protein degradation is not dependent on changes in mitochondrial morphology and increased FAO. We went further to determine whether inhibiting PDGF-induced 
autophagy (or contractile protein degradation) would affect mitochondrial fragmentation. Also, Spautin1, an inhibitor of autophagy, did not inhibit PDGF induced fragmentation. These data suggest that contractile protein degradation and mitochondria fragmentation occur via separate pathways. The relative contributions of these pathways lead to the emergence of a fully dedifferentiated VSMC characterized by decreased expression of smooth muscle cell contractile proteins, increased proliferation and other synthetic characteristics such as increased migration and ECM synthesis (see Scheme 6).

\section{Discussion}

Our present study shows that mitochondrial morphology is important for regulating substrate usage and VSMC proliferation in the synthetic phenotype. We demonstrated that conversion of VSMCs into the synthetic phenotype is accompanied by increased FAO which is preceded by mitochondrial fragmentation. This change in mitochondria morphology appears to be required for increasing FAO and proliferation, as mdivi1 inhibited both mitochondrial fragmentation and proliferation. We also found that although the change in substrate metabolism was required for increased proliferation, it did not have any effect on PDGF-induced contractile protein degradation. These findings uncover mitochondrial fragmentation as one of many extensive cellular changes occurring during conversion into the synthetic phenotype. 
Beta oxidation of long chain fatty acids occurs in the mitochondria and yields two times more ATP per gram when compared with glucose or amino acids [250251]. Thus, under conditions of excessive energy demand, cells are more likely benefit from FAO. Since long chain FAs cannot freely diffuse through the mitochondrial inner membrane, a transport system is required. This transport system comprises a group of enzymes known as carnitine palmitoyl transferases (CPT) [252]. CPT1, the rate limiting enzyme of the CPT system [252], catalyses the transfer of an acyl group from coenzyme $A$ on an activated FA to carnitine to form palmitoylcarnitine. Palmitoylcarnitine is then shuttled across the inner mitochondria membrane where it is converted back into palmitoyl-CoA. PalmitoylCoA can be broken down to acetyl-CoA which enters the TCA cycle to yield ATP and the reducing equivalents $\mathrm{NADH}$ and $\mathrm{FADH}_{2}$ [253]. We found that etomoxir, an inhibitor of CPT1, inhibited PDGF-induced VSMC proliferation but not mitochondria fragmentation.

We proposed that changes in mitochondria morphology induced by PDGF regulate substrate utilization. Changes in substrate metabolism could lead to increased glycolysis (resulting in increased flux of pyruvate into mitochondria) or could lead to increased FA utilization or both. Interestingly, we found that in addition to increased glycolysis after PDGF stimulation of VSMCs, FAO was also increased. These combined increases in glycolysis and FAO may be important for supporting energy requirements and cellular changes in the fully synthetic phenotype. 
Previous studies in other cell types show that promotion of mitochondrial fission by knock down of Mfn2 leads to reduced mitochondrial membrane potential, less oxidative phosphorylation and increased dependency on glycolysis, possibly mediated through reduced expression and activity of different subunits of the electron transport chain [254]. In the case of PDGF-induced mitochondrial fragmentation, we found that the overall oxidative phosphorylation of both contractile and synthetic cells remained the same and there were no changes in expression of the subunits in the electron transport chain (Figure 19).

Mitochondria fission was found to be important in PDGF-induced proliferation through a mechanism involving regulation of expression of cell cycle proteins such as PCNA and cyclin D1. In fact, in the presence of Mdivi1, the expression of PCNA and cyclin D1 were inhibited and cells became stalled in the S-phase. However, it remains to be clearly elucidated whether the effects of Mdivi1 on proliferation are linked to its mitochondria-fission-inhibitory effect or due to a different effect. It is however important to note that the effects on proliferation observed with Mdivi1 concur previous findings showing that promotion of mitochondria fusion through overexpression of Mfn2 promote inhibition of VSMC proliferation [244].

Collectively, these studies provide novel insights into the role of mitochondria in PDGF-induced proliferation. Mitochondria morphology is a determinant of substrate usage but not of protein degradation during phenotype switching. Identification of the role of mitochondria in VSMC opens new avenues for therapeutic management of atherosclerosis and restenosis. 
Figure 22. PDGF-induced mitochondrial fragmentation results in increased

FAO. Inhibition of mitochondria fission inhibits PDGF-induced fragmentation and

FAO. A) Representative confocal images of serum starved VSMCs treated without or with PDGF \pm Mdivi-1. Morphology is shifted towards the filamentous in the presence of Mdivi1. B) Quantification of A. Fil = filamentous, Glo = Globular and Int $=$ Intermediate. $(\geq 150$ cells counted per experiment, experiment was repeated at least two times). C) Representative extracellular flux measurements. VSMCs were serum starved for $24 \mathrm{~h}$ and then left untreated or converted to the synthetic phenotype by PDGF treatment for $48 \mathrm{~h} \pm$ Mdivi1. D) Quantification of C ( $n=4$ independent experiments, ${ }^{*} p<0.05$ vs ctrl, ${ }^{8} p<0.05$ vs PDGF). 
Figure 22

A
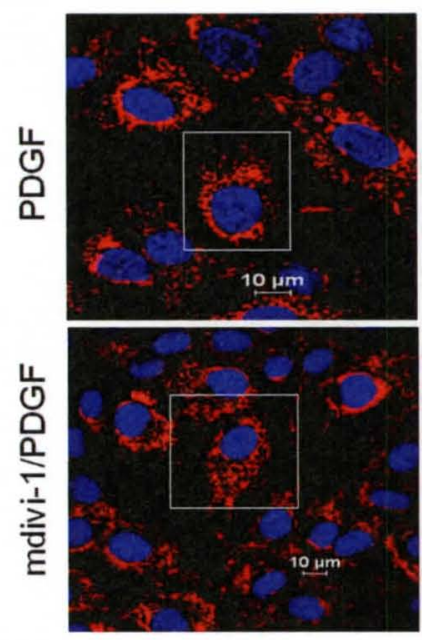

C

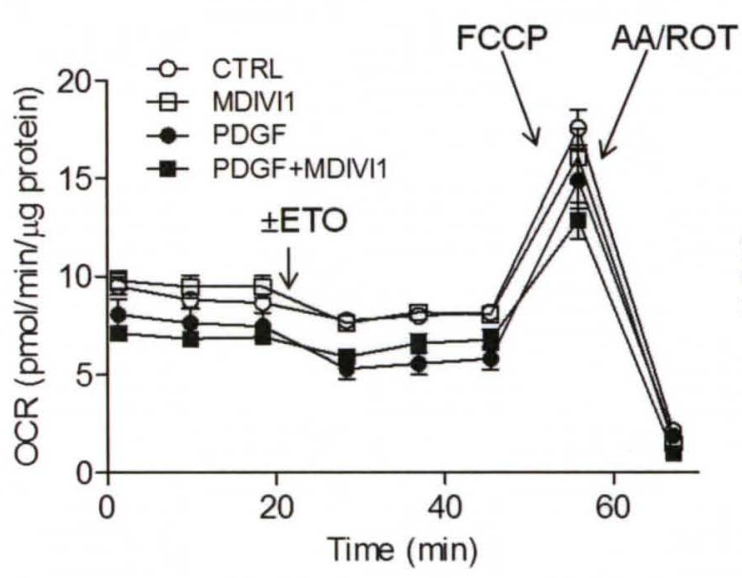

B
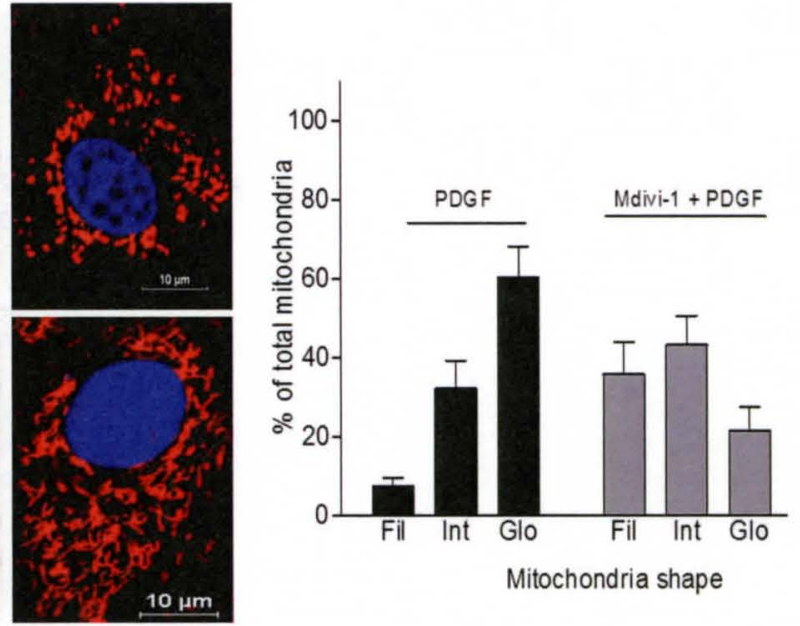

D

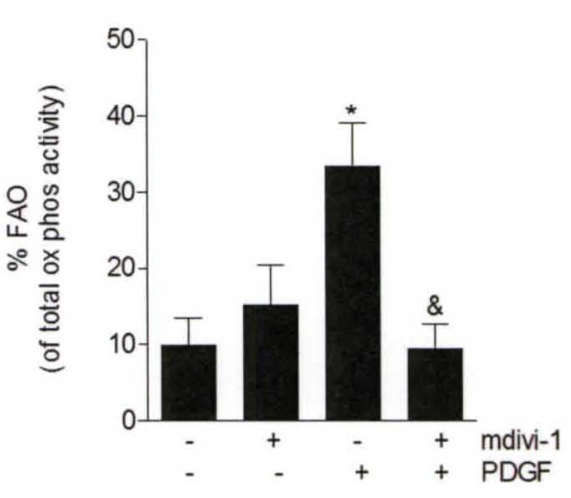


Figure 23. Inhibition of mitochondrial fragmentation decreases PDGFinduced proliferation. A) Cell proliferation after PDGF \pm mdivi1 treatment. VSMCs were serum starved for $24 \mathrm{~h}$ and then treated for $24 \mathrm{~h}(\mathrm{n}=5$ per treatment group, ${ }^{*} p<0.03$ vs control, ${ }^{\&} p<0.01$ vs PDGF). B) Inhibition of cell cycle protein expression by mdivi1. VSMCs were serum starved for $24 \mathrm{~h}$ and then stimulated with PDGF for the indicated times in the absence or presence of mdivi1. C and D) Quantification of $B\left(n=3\right.$ per treatment group, ${ }^{*} p<0.05$ vs untreated). E) Cell cycle analysis using propidium iodide. Mdivi1 stalls cells in the S-phase of cell cycle. Cells in upper panel were stimulated with PDGF for the indicated times while cells in the lower panel were stimulated with PDGF but in the presence of mdivi1. 
Figure 23
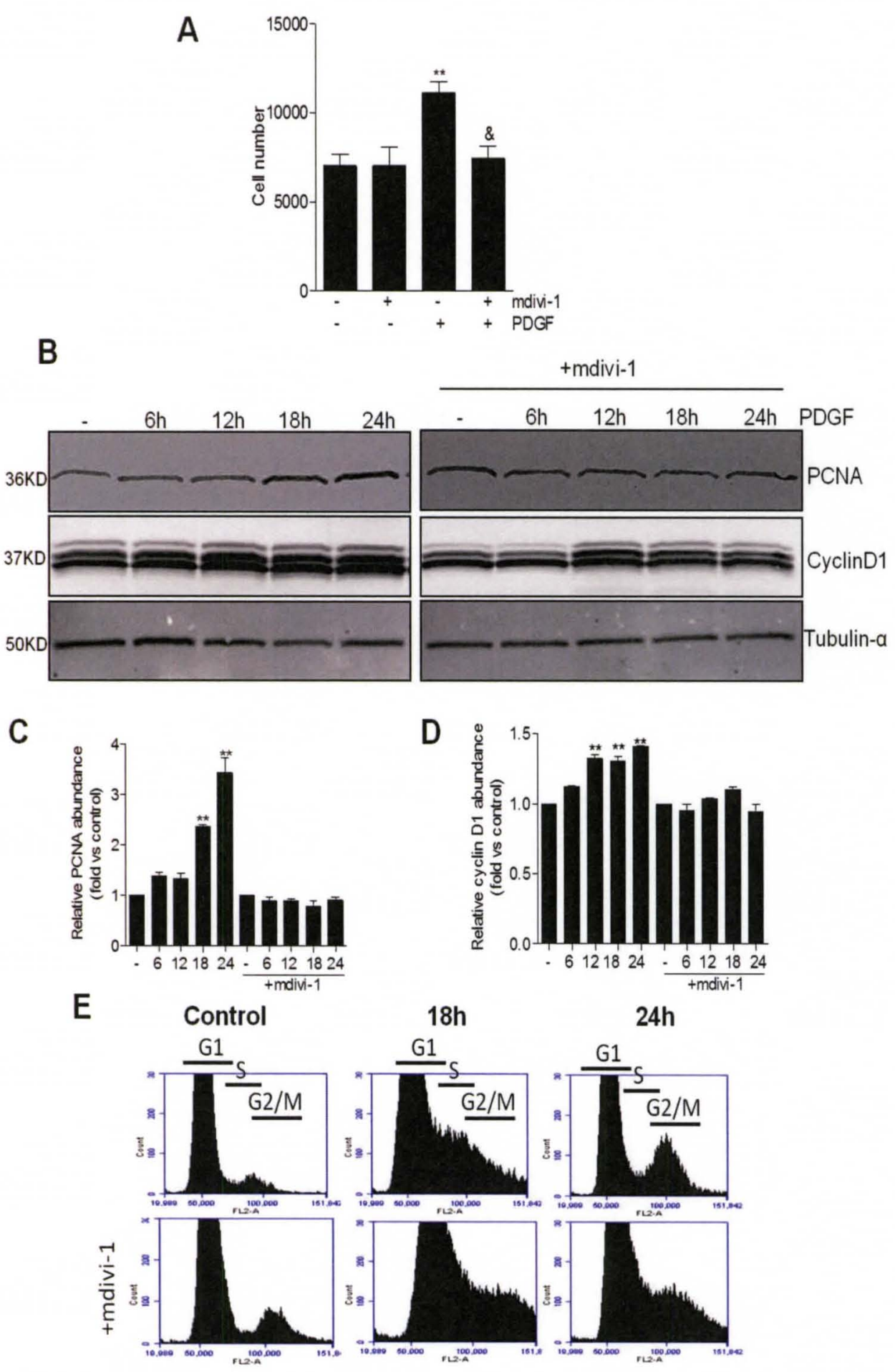
Figure 24. FAO is required for PDGF-induced proliferation. Etomoxir inhibits PDGF-induced proliferation. A) Concentration dependent effect of etomoxir on VSMC proliferation. Cell proliferation was measured by counting cell numbers using the Accuri $\mathrm{C} 6$ flow cytometer ( $n=6,{ }^{*} p<0.05$ vs untreated). B) Cell viability by MTT assay. VSMCs after exposure to different concentrations of etomoxir ( $n=6,{ }^{* * *} \mathrm{P}<0.01$ vs control). C) Effects of $\mathrm{FAO}$ on mitochondria fragmentation. 
Figure 24

A

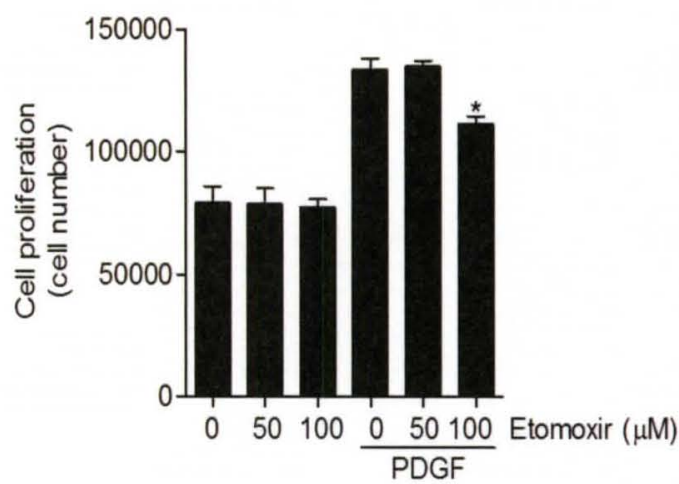

C
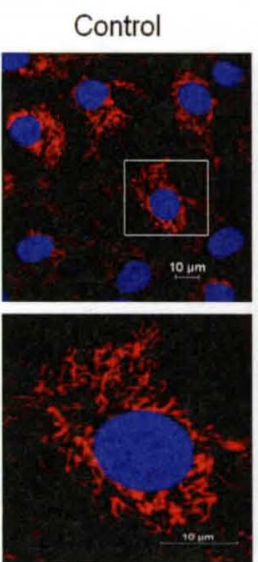

Etomoxi
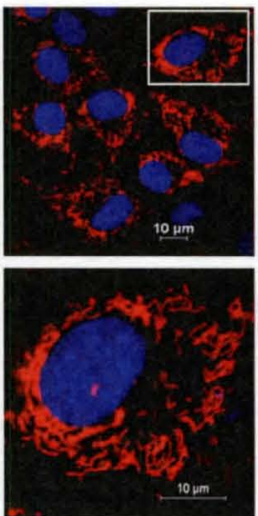

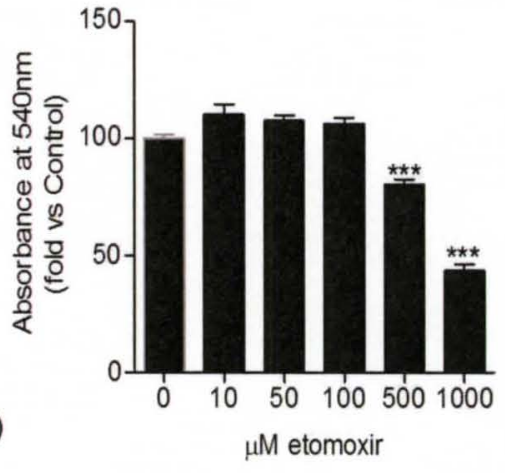

PDGF

Etomoxir/PDGF
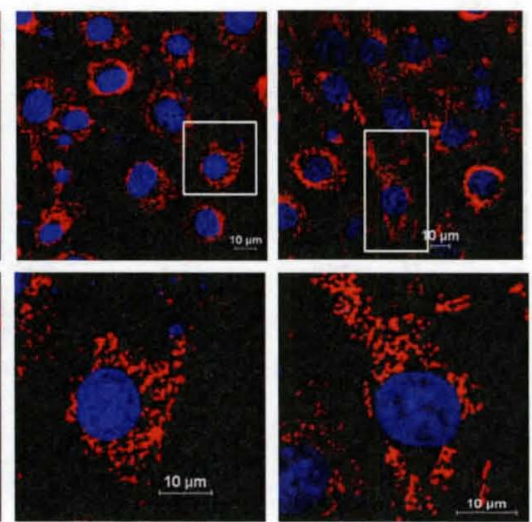
Figure 25. Mitochondria fragmentation and FAO are not prerequisites for PDGF-induced contractile protein degradation. Mdivi1 does not inhibit PDGFinduced autophagy and contractile protein degradation. A) Representative immunoblot after PDGF \pm mdivi1 treatment. Cells were serum starved for $24 \mathrm{~h}$ before treatment. B-D) Quantification of $A\left(n=3,{ }^{*} P<0.05,{ }^{* *} p<0.01\right.$ vs untreated). E) FAO is not required for PDGF-induced autophagy and contractile protein degradation. Representative immunoblot after PDGF \pm etomoxir treatment. 
Figure 25

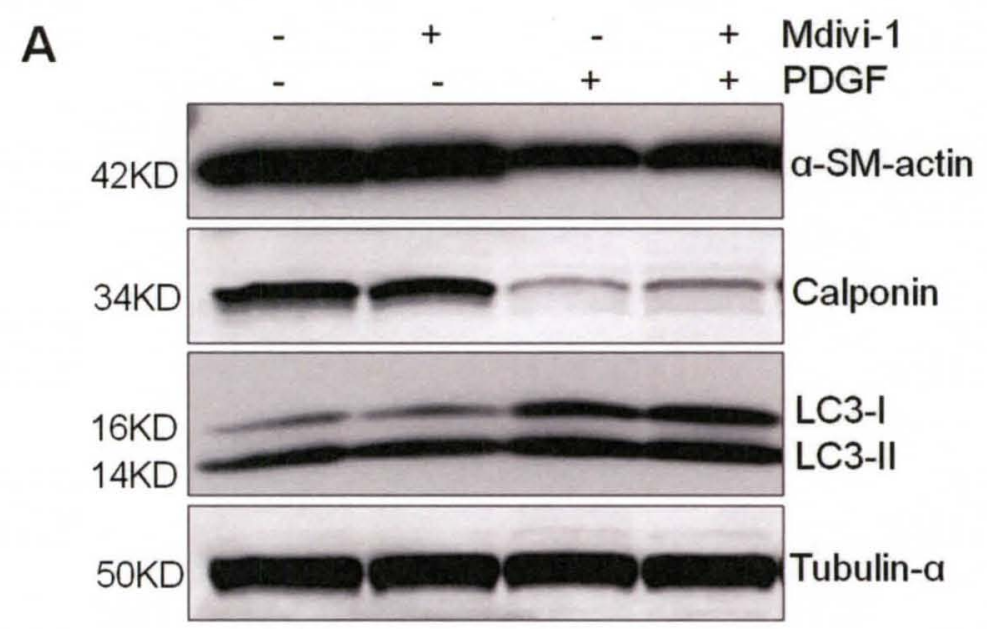

B

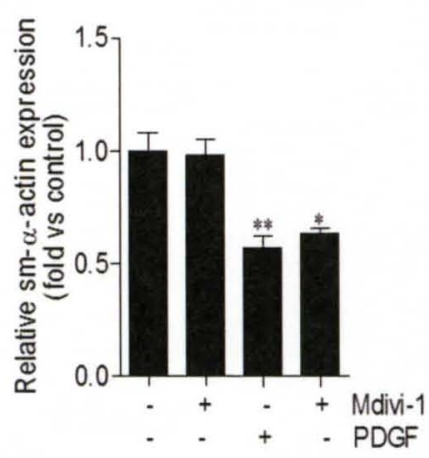

C

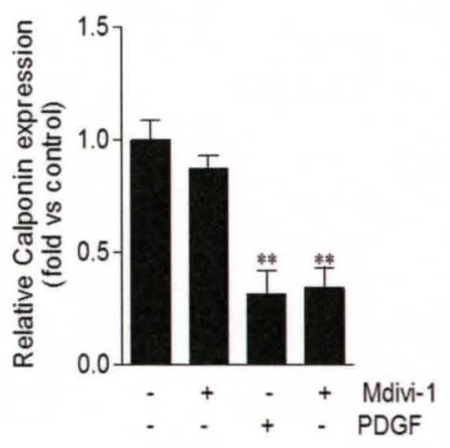

D

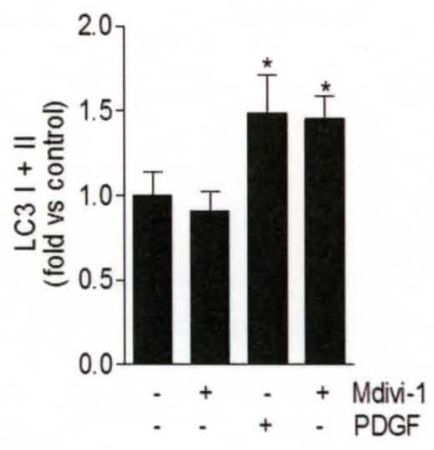

E

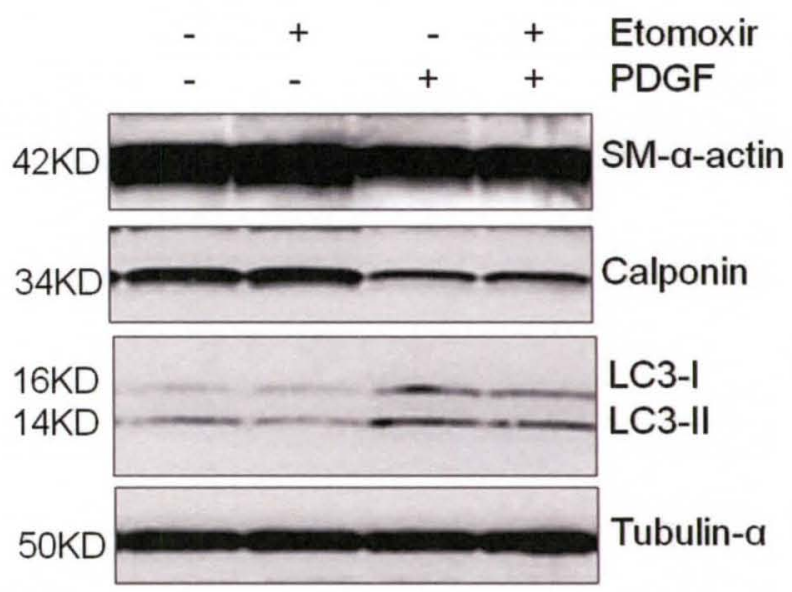




\section{CONCLUDING DISCUSSION}

Our studies were aimed at developing a better understanding of how metabolism regulates VSMC phenotype. For this, we examined processes relating to protein degradation and studied metabolic changes occurring during and after VSMC phenotype transition. We proposed that in addition to inducing other cellular processes needed for the conversion to the synthetic phenotype, PDGF induces autophagy as a means to degrade unwanted cellular components, making way for the emergence of a fully synthetic phenotype. We also proposed that PDGF stimulation regulates cellular metabolism to increase energy production which supports proliferation and synthetic VSMC activity.

In the first set of experiments we wanted to determine which protein degradation pathways are primarily involved during phenotype switching. We examined the proteasomal and the autophagic pathways - the predominant pathways of cellular degradation in mammalian cells. We found that inhibition of the

proteasomal pathway had limited effects on 
PDGF-induced degradation of calponin and sm- $\alpha$-actin. This suggested to us that the autophagic pathway of protein degradation could be involved during phenotypic conversion.

The conversion to the synthetic phenotype requires rapid cellular changes and degradation of contractile proteins, which would be thought to require proteolytic pathways such as autophagy or the proteasome. In mammalian cells, autophagy is known to be the major pathway through which cellular contents are degraded during growth and transformation. We therefore examined the role of autophagy during phenotype transition. In chapter II, we presented data showing that PDGF promotes autophagy in VSMCs. The induction of autophagy by a mitogen is atypical, because other growth factors (e.g., insulin) have been shown to inhibit, rather than activate, autophagy. PDGF-induced autophagy appears to be important in phenotype switching because inhibition of autophagy with different structural inhibitors led to stabilization of calponin and sm- $\alpha$-actin. This appears to be specific to PDGF because other mitogens such as FGF-1 neither induced autophagy nor promoted phenotypic switching.

Interestingly, PDGF-induced autophagy was protective against HNE-induced toxicity. This is consistent with multiple studies showing that autophagy is a survival response activated during starvation and under conditions of oxidative stress. Because HNE is a constituent of atherosclerotic lesions known to form protein-HNE adducts and to cause cytotoxicity, the heightened autophagy in synthetic cells could render these cells stress resistant hence enhancing plaque 
stability during atherogenesis. Our findings therefore support the notion that (mitogen-mediated) autophagic induction in VSMCs could be protective, therefore exposing new therapeutic avenues. However, in the context of atherosclerosis, the manipulation of autophagy for therapeutic success should be approached with caution because induction of autophagy can be maladaptive as well. For example, excessive induction of autophagy might eventually trigger autophagy-induced cell death or type 2 cell death, resulting in increased plaque destabilization [255].

The induction of autophagy during phenotypic conversion may also be advantageous to the rapidly proliferating synthetic cell. Since autophagy degrades cellular components to their constituent amino acids, these could be used as building blocks for different and new macromolecules prevalent in the synthetic cells. The importance of autophagy in this regard could be seen from the findings that inhibiting autophagy decreased VSMC proliferation, a key feature of the synthetic phenotype. Our findings therefore suggest a role of autophagy in VSMC proliferation. This novel role could also be exploited for therapeutic advantage in vasculo-proliferative complications such as restenosis [27].

In chapter III, we found that an increase in glycolytic flux after PDGF treatment does not only increase mitochondrial activity [145], but it is also required for the expression of the synthetic gene OPN. OPN is a cytokine secreted by synthetic 
VSMCs and it is known to promote plaque formation by promoting VSMC migration into intima. Our findings therefore suggest a possible link between glycolysis and vascular lesions caused by VSMC hyperproliferation. Our findings also confirm previous findings implicating the role of glucose-regulated transcription factors in OPN expression.

PDGF-induced OPN expression was also partly regulated by O-GIcNAc formation. Increasing O-GIcNAc formation via Thiamet-g treatment inhibited PDGF-induced OPN expression. This finding supports previous findings suggesting an inhibitory role of increased HBP flux (or O-GIcNAc) in VSMC hyperproliferation. However, in hyperglycemic conditions such diabetes, increased flux into the HBP has been thought to contribute to VSMC hyperproliferation and has been associated with an increased incidence of CVD. Our results therefore suggest the contrary because increased levels of O-GIcNAc (an end product of increased HBP flux) had inhibitory effects on VSMCs. However, it is worth noting that increased flux to the HBP does not necessarily imply an increase in (protein) O-GlcNAc formation. Also, the diabetic condition is not only characterized by increased glucose flux into the HBP, but also by other conditions such as increased levels of circulating free fatty acids.

The increased O-GlcNAc formation observed after Thiamet-g treatment was associated with delay in cell cycle progression and decrease expression of cell cycle proteins cyclin D1 and PCNA. Thiamet-g-treated cells were stalled between the G1 and S-phases of cell cycle. Since O-GlcNAc modification is a 
posttranslational modification, the inhibitory effects of this modification on cell cycle proteins could cause cell cycle arrest. It remains to be determined whether O-GIcNAc has a direct modifying effect on cell cycle proteins or its effects on cell cycle progression are mediated via other intermediary proteins. Furthermore, more experiments are needed to rigorously test the contribution of the HBP and protein O-GlcNAc formation in vascular complications under normal and diabetic conditions.

In chapter IV, we found that intermediary metabolism plays an important role in PDGF-induced phenotype transition. Specifically, we found that synthetic VSMCs show increased FAO and decreased rates of glucose oxidation (described in chapter III). This increase in FAO was preceded by changes in mitochondrial morphology. The increases in FAO may be required to meet the high energy demands of synthetic cells.

In the synthetic state, VSMCs had mostly rounded or fragmented mitochondria compared with the long filamentous mitochondria found in contractile cells. It has been previously reported that filamentous mitochondria form giant networks that are associated with increased ATP production and that these networks can evade autophagic degradation under conditions of starvation. Here, however, we found that the fragmented mitochondrial phenotype may instead be associated with increased ATP production (since it utilizes more fatty acids). This increase in FAO in synthetic cells could be as a result of increased total mitochondria 
surface area or increased expression (or activation) of proteins involved in FAO such as CPT1. The fragmented mitochondria (as well as increased FAO) contributed to VSMC hyperplasia because mdivi 1 (which promotes fusion) inhibited proliferation. These findings are consistent with previous studies showing that promoting mitochondrial fusion in VSMCs, by overexpressing the pro-fusion gene mitofusin, prevents VSMC hyperplasia, and that the fragmented mitochondria are predominant in diseased VSMCs such as found in hypertensive vessels $[245,256]$. Inhibition of mitochondrial fragmentation also prevented increased FAO. This suggests that mitochondrial fragmentation is upstream of FAO observed upon PDGF stimulation.

During mitosis, mitochondria fragmentation is required to produce equal mitochondria numbers for the emerging daughter cells. We found that inhibition of mitochondrial fragmentation caused cells to stall in the S-phase leading to inhibition of cell proliferation. This inhibition of cell proliferation could be attributed to: 1) the unavailability of mitochondria for the prospective daughter cells; and 2) the inhibition of FAO caused by mitochondrial fragmentation. In chapter IV, we present data supporting the latter because inhibiting FAO led to inhibition of cell proliferation.

In summary, our results uncover novel mechanisms by which metabolism regulates VSMC phenotype. Our data identify autophagy as an important process regulating phenotypic switching and provide new insights into the role of 
fatty acid metabolism in phenotypic transition. Although the metabolic and autophagic changes during this transition seem to occur via separate pathways, these pathways contribute to the emergence of a fully synthetic phenotype (Scheme 6). These data could form the basis for of the development of more effective anti-restenotic and -atherosclerotic therapies. Nevertheless, future studies are required to decipher the mechanism(s) involved in our observations. Studies aimed at determining the contributions of other substrates such as glutamine also warrant our attention, and in vivo studies are required to define the usefulness of chemical regulators of autophagy, O-GIcNAc and mitochondrial morphology on VSMC proliferation. 


\section{Scheme 6}

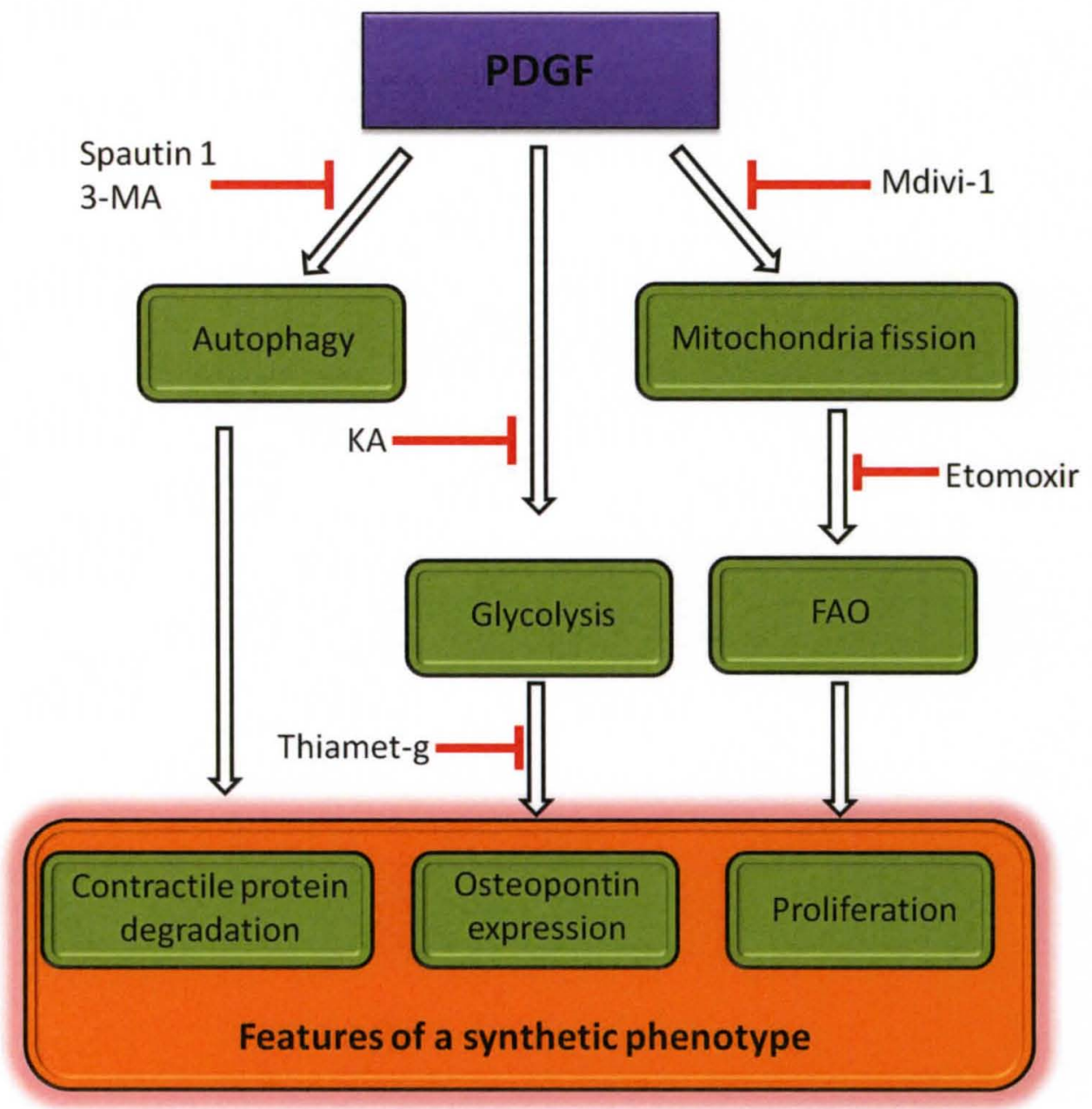

Regulation of phenotype switching by autophagy and intermediary metabolism 


\section{REFERENCES}

1. Rosamond, W., et al., Heart disease and stroke statistics - 2008 update - A report from the American Heart Association Statistics Committee and Stroke Statistics Subcommittee. Circulation, 2008. 117(4): p. E25-E146.

2. Roger, Heart Disease and Stroke Statistics-2011 Update: A Report From the American Heart Association (vol 123, pg e18, 2011). Circulation, 2011. 123(6): p. E240-E240.

3. Wang, Z.V., B.A. Rothermel, and J.A. Hill, Autophagy in hypertensive heart disease. J Biol Chem, 2010. 285(12): p. 8509-14.

4. Baseline characteristics of patients with diabetes and coronary artery disease enrolled in the Bypass Angioplasty Revascularization Investigation 2 Diabetes (BARI 2D) trial. Am Heart J, 2008. 156(3): p. 528-536, 536 e1-5.

5. Mendis, S., et al., Global atlas on cardiovascular disease prevention and control. 2011, Geneva: World Health Organization in collaboration with the World Heart Federation and the World Stroke Organization. vi, 155 p.

6. Rosamond, Heart Disease and Stroke Statistics-2008 Update: A Report From the American Heart Association Statistics Committee and Stroke Statistics Subcommittee (vol 117, pg e25, 2008). Circulation, 2010. 122(1): p. E10-E10.

7. Ross, R., The Pathogenesis of Atherosclerosis - a Perspective for the 1990s. Nature, 1993. 362(6423): p. 801-809.

8. Ait-Oufella, H., A. Tedgui, and Z. Mallat, Atherosclerosis: an inflammatory disease. Sang Thrombose Vaisseaux, 2008. 20(1): p. 25-33.

9. Mensah, G.A. and D.W. Brown, An overview of cardiovascular disease burden in the United States. Health Aff (Millwood), 2007. 26(1): p. 38-48.

10. Damon, D.H., Sympathetic innervation promotes vascular smooth muscle differentiation. American Journal of Physiology-Heart and Circulatory Physiology, 2005. 288(6): p. $\mathrm{H} 2785-\mathrm{H} 2791$.

11. van der Velden, V.H.J. and A.R. Hulsmann, Autonomic innervation of human airways: Structure, function, and pathophysiology in asthma. Neuroimmunomodulation, 1999. 6(3): p. 145-159.

12. Nagy, J.A., et al., VEGF-A induces angiogenesis, arteriogenesis, lymphangiogenesis, and vascular malformations. Cold Spring Harb Symp Quant Biol, 2002. 67: p. 227-37.

13. Carmeliet, P., Mechanisms of angiogenesis and arteriogenesis. Nat Med, 2000. 6(4): p. 389-95.

14. Carmeliet, P., Mechanisms of angiogenesis and arteriogenesis. Nature Medicine, 2000. 6(4): p. 389-95.

15. Hall, A.P., Review of the pericyte during angiogenesis and its role in cancer and diabetic retinopathy. Toxicol Pathol, 2006. 34(6): p. 763-75.

16. McGrath, J.C., et al., New aspects of vascular remodelling: the involvement of all vascular cell types. Experimental Physiology, 2005. 90(4): p. 469-475. 
17. Kantachuvesiri, S., et al., Controlled hypertension, a transgenic toggle switch reveals differential mechanisms underlying vascular disease. Journal of Biological Chemistry, 2001. 276(39): p. 36727-33.

18. Glass, C.K. and J.L. Witztum, Atherosclerosis. the road ahead. Cell, 2001. 104(4): p. 50316.

19. Hansson, G.K. and P. Libby, The immune response in atherosclerosis: a double-edged sword. Nat Rev Immunol, 2006. 6(7): p. 508-19.

20. Koga, J. and M. Aikawa, Crosstalk between macrophages and smooth muscle cells in atherosclerotic vascular diseases. Vascular Pharmacology, 2012. 57(1): p. 24-28.

21. Allahverdian, S., P.S. Pannu, and G.A. Francis, Contribution of monocyte-derived macrophages and smooth muscle cells to arterial foam cell formation. Cardiovascular Research, 2012. 95(2): p. 165-172.

22. Doran, A.C., N. Meller, and C.A. McNamara, Role of smooth muscle cells in the initiation and early progression of atherosclerosis. Arteriosclerosis Thrombosis and Vascular Biology, 2008. 28(5): p. 812-819.

23. Newby, A.C. and A.B. Zaltsman, Fibrous cap formation or destruction--the critical importance of vascular smooth muscle cell proliferation, migration and matrix formation. Cardiovasc Res, 1999. 41(2): p. 345-60.

24. Tousoulis, D., et al., Insight to the pathophysiology of stable angina pectoris. Curr Pharm Des, 2012.

25. Aronson, D. and E.R. Edelman, Revascularization for coronary artery disease in diabetes mellitus: Angioplasty, stents and coronary artery bypass grafting. Reviews in Endocrine \& Metabolic Disorders, 2010. 11(1): p. 75-86.

26. Newby, A.C. and A.B. Zaltsman, Molecular mechanisms in intimal hyperplasia. Journal of Pathology, 2000. 190(3): p. 300-309.

27. Billinger, M., et al., Long-term clinical and angiographic outcomes of diabetic patients after revascularization with early generation drug-eluting stents. American Heart Journal, 2012. 163(5): p. 876--.

28. Kawai-Kowase, K. and G.K. Owens, Multiple repressor pathways contribute to phenotypic switching of vascular smooth muscle cells. American Journal of Physiology-Cell Physiology, 2007. 292(1): p. C59-C69.

29. Van Belle, E., et al., Restenosis rates in diabetic patients: a comparison of coronary stenting and balloon angioplasty in native coronary vessels. Circulation, 1997. 96(5): p. 1454-60.

30. Elezi, S., et al., Diabetes mellitus and the clinical and angiographic outcome after coronary stent placement. J Am Coll Cardiol, 1998. 32(7): p. 1866-73.

31. Schofer, J., et al., Influence of treatment modality on angiographic outcome after coronary stenting in diabetic patients: a controlled study. J Am Coll Cardiol, 2000. 35(6): p. 1554-9.

32. Van Belle, E., et al., Restenosis, late vessel occlusion and left ventricular function six months after balloon angioplasty in diabetic patients. J Am Coll Cardiol, 1999. 34(2): p. 476-85.

33. Kip, K.E., et al., Coronary angioplasty in diabetic patients. The National Heart, Lung, and Blood Institute Percutaneous Transluminal Coronary Angioplasty Registry. Circulation, 1996. 94(8): p. 1818-25.

34. Stein, B., et al., Influence of diabetes mellitus on early and late outcome after percutaneous transluminal coronary angioplasty. Circulation, 1995. 91(4): p. 979-89. 
35. Mak, K.H. and D.P. Faxon, Clinical studies on coronary revascularization in patients with type 2 diabetes. Eur Heart J, 2003. 24(12): p. 1087-103.

36. Owens, G.K., M.S. Kumar, and B.R. Wamhoff, Molecular regulation of vascular smooth muscle cell differentiation in development and disease. Physiol Rev, 2004. 84(3): p. 767801.

37. Gabbiani, G., et al., Vascular smooth muscle cells differ from other smooth muscle cells: predominance of vimentin filaments and a specific alpha-type actin. Proc Natl Acad Sci U S A, 1981. 78(1): p. 298-302.

38. Hungerford, J.E., et al., Development of the aortic vessel wall as defined by vascular smooth muscle and extracellular matrix markers. Developmental Biology, 1996. 178(2): p. 375-392.

39. Mack, C.P. and G.K. Owens, Regulation of smooth muscle alpha-actin expression in vivo is dependent on CArG elements within the $5^{\prime}$ and first intron promoter regions. Circ Res, 1999. 84(7): p. 852-61.

40. Madsen, C.S., et al., Smooth muscle-specific expression of the smooth muscle myosin heavy chain gene in transgenic mice requires 5 '-flanking and first intronic DNA sequence. Circ Res, 1998. 82(8): p. 908-17.

41. Miano, J.M., et al., Smooth muscle myosin heavy chain exclusively marks the smooth muscle lineage during mouse embryogenesis. Circ Res, 1994. 75(5): p. 803-12.

42. Duband, J.L., et al., Calponin and SM 22 as differentiation markers of smooth muscle: spatiotemporal distribution during avian embryonic development. Differentiation, 1993. 55(1): p. 1-11.

43. Miano, J.M., et al., Serum response factor-dependent regulation of the smooth muscle calponin gene. J Biol Chem, 2000. 275(13): p. 9814-22.

44. Kim, S., et al., A serum response factor-dependent transcriptional regulatory program identifies distinct smooth muscle cell sublineages. Mol Cell Biol, 1997. 17(4): p. 2266-78.

45. van der Loop, F.T., et al., Smoothelin, a novel cytoskeletal protein specific for smooth muscle cells. J Cell Biol, 1996. 134(2): p. 401-11.

46. Li, L., et al., SM22 alpha, a marker of adult smooth muscle, is expressed in multiple myogenic lineages during embryogenesis. Circ Res, 1996. 78(2): p. 188-195.

47. Perry, R.L.S. and M.A. Rudnicki, Molecular mechanisms regulating myogenic determination and differentiation. Frontiers in Bioscience, 2000. 5: p. D750-D767.

48. Giachelli, C.M., et al., Osteopontin Is Elevated during Neointima Formation in Rat Arteries and Is a Novel Component of Human Atherosclerotic Plaques. Journal of Clinical Investigation, 1993. 92(4): p. 1686-1696.

49. Yoshida, T. and G.K. Owens, Molecular determinants of vascular smooth muscle cell diversity. Circ Res, 2005. 96(3): p. 280-91.

50. Chen, C.N., et al., Synergistic roles of platelet-derived growth factor-BB and interleukin1 beta in phenotypic modulation of human aortic smooth muscle cells. Proc Natl Acad Sci U S A, 2006. 103(8): p. 2665-70.

51. Ichii, T., et al., Fibrillar collagen specifically regulates human vascular smooth muscle cell genes involved in cellular responses and the pericellular matrix environment. Circ Res, 2001. 88(5): p. 460-7.

52. Hedin, U., et al., Diverse effects of fibronectin and laminin on phenotypic properties of cultured arterial smooth muscle cells. Journal of Cell Biology, 1988. 107(1): p. 307-19.

53. Pickering, J.G., Regulation of vascular cell behavior by collagen - Form is function. Circulation Research, 2001. 88(5): p. 458-459. 
54. Wang, L., et al., Cartilage Oligomeric Matrix Protein Maintains the Contractile Phenotype of Vascular Smooth Muscle Cells by Interacting With alpha(7)beta(1) Integrin. Circulation Research, 2010. 106(3): p. 514-525.

55. $\mathrm{Li}, \mathrm{X}$, et al., Suppression of smooth-muscle alpha-actin expression by platelet-derived growth factor in vascular smooth-muscle cells involves Ras and cytosolic phospholipase A2. Biochem J, 1997. 327 ( Pt 3): p. 709-16.

56. Holycross, B.J., et al., Platelet-derived growth factor-BB-induced suppression of smooth muscle cell differentiation. Circ Res, 1992. 71(6): p. 1525-32.

57. Fredriksson, L., H. Li, and U. Eriksson, The PDGF family: four gene products form five dimeric isoforms. Cytokine Growth Factor Rev, 2004. 15(4): p. 197-204.

58. Yoshida, T., et al., Platelet-derived growth factor-BB represses smooth muscle cell marker genes via changes in binding of MKL factors and histone deacetylases to their promoters. American Journal of Physiology-Cell Physiology, 2007. 292(2): p. C886-C895.

59. Dandre, F. and G.K. Owens, Platelet-derived growth factor-BB and Ets-1 transcription factor negatively regulate transcription of multiple smooth muscle cell differentiation marker genes. American Journal of Physiology-Heart and Circulatory Physiology, 2004. 286(6): p. H2042-H2051.

60. Kato, S., et al., Basic fibroblast growth factor regulates extracellular matrix and contractile protein expression independent of proliferation in vascular smooth muscle cells. In Vitro Cellular \& Developmental Biology-Animal, 1998. 34(4): p. 341-346.

61. Millette, E., et al., Platelet-derived growth factor-BB-induced human smooth muscle cell proliferation depends on basic FGF release and FGFR-1 activation. Circulation Research, 2005. 96(2): p. 172-9.

62. Perlegas, D., et al., ANG // type 2 receptor regulates smooth muscle growth and force generation in late fetal mouse development. American Journal of Physiology-Heart and Circulatory Physiology, 2005. 288(1): p. H96-H102.

63. Deaton, R.A., et al., Transforming growth factor-beta1-induced expression of smooth muscle marker genes involves activation of PKN and p38 MAPK. Journal of Biological Chemistry, 2005. 280(35): p. 31172-81.

64. Long, X. and J.M. Miano, Transforming growth factor-beta1 (TGF-beta1) utilizes distinct pathways for the transcriptional activation of microRNA 143/145 in human coronary artery smooth muscle cells. Journal of Biological Chemistry, 2011. 286(34): p. 30119-29.

65. Zhao, Y., et al., PDGF-induced vascular smooth muscle cell proliferation is associated with dysregulation of insulin receptor substrates. American Journal of Physiology-Cell Physiology, 2011. 300(6): p. C1375-C1385.

66. Pidkovka, N.A., et al., Oxidized phospholipids induce phenotypic switching of vascular smooth muscle cells in vivo and in vitro. Circulation Research, 2007. 101(8): p. 792-801.

67. Cherepanova, O.A., et al., Oxidized Phospholipids Induce Type VIII Collagen Expression and Vascular Smooth Muscle Cell Migration. Circulation Research, 2009. 104(5): p. 609U112.

68. Clempus, R.E., et al., Nox4 is required for maintenance of the differentiated vascular smooth muscle cell phenotype. Arteriosclerosis Thrombosis and Vascular Biology, 2007. 27(1): p. 42-48.

69. Davis, R., et al., TNF-alpha-mediated proliferation of vascular smooth muscle cells involves Raf-1-mediated inactivation of $R b$ and transcription of E2F1-regulated genes. Cell Cycle, 2012. 11(1): p. 109-18.

70. Wang, Y.S., et al., MicroRNA-195 regulates vascular smooth muscle cell phenotype and prevents neointimal formation. Cardiovasc Res, 2012. 95(4): p. 517-26. 
71. Cheng, Y., et al., MicroRNA-145, a novel smooth muscle cell phenotypic marker and modulator, controls vascular neointimal lesion formation. Circulation Research, 2009. 105(2): p. 158-66.

72. Cordes, K.R., et al., miR-145 and miR-143 regulate smooth muscle cell fate and plasticity. Nature, 2009. 460(7256): p. 705-10.

73. Kuhn, A.R., et al., MicroRNA expression in human airway smooth muscle cells: role of miR-25 in regulation of airway smooth muscle phenotype. Am J Respir Cell Mol Biol, 2010. 42(4): p. 506-13.

74. Chen, J., et al., Induction of microRNA-1 by myocardin in smooth muscle cells inhibits cell proliferation. Arterioscler Thromb Vasc Biol, 2011. 31(2): p. 368-75.

75. Liu, X., et al., A necessary role of miR-221 and miR-222 in vascular smooth muscle cell proliferation and neointimal hyperplasia. Circulation Research, 2009. 104(4): p. 476-87.

76. Ji, R., et al., MicroRNA expression signature and antisense-mediated depletion reveal an essential role of MicroRNA in vascular neointimal lesion formation. Circulation Research, 2007. 100(11): p. 1579-88.

77. Hedin, U., et al., A Substrate of the Cell-Attachment Sequence of Fibronectin (Arg-GlyAsp-Ser) is Sufficient to Promote Transition of Arterial Smooth-Muscle Cells from a Contractile to a Synthetic Phenotype. Developmental Biology, 1989. 133(2): p. 489-501.

78. Pauly, R.R., et al., Experimental-Models That Mimic the Differentiation and Dedifferentiation of Vascular Cells. Circulation, 1992. 86(6): p. 68-73.

79. Wang, L., et al., ADAMTS-7 mediates vascular smooth muscle cell migration and neointima formation in balloon-injured rat arteries. Circulation Research, 2009. 104(5): p. 688-98.

80. Wang, L., et al., Cartilage oligomeric matrix protein maintains the contractile phenotype of vascular smooth muscle cells by interacting with alpha(7)beta(1) integrin. Circulation Research, 2010. 106(3): p. 514-25.

81. Gao, H., M.C. Steffen, and K.S. Ramos, Osteopontin regulates alpha-smooth muscle actin and calponin in vascular smooth muscle cells. Cell Biol Int, 2012. 36(2): p. 155-61.

82. Martin, K.A., et al., The $m T O R / p 70$ S6K1 pathway regulates vascular smooth muscle cell differentiation. American Journal of Physiology-Cell Physiology, 2004. 286(3): p. C507C517.

83. Martin, K.A., et al., Rapamycin promotes vascular smooth muscle cell differentiation through insulin receptor substrate-1/phosphatidylinositol 3-kinase/Akt2 feedback signaling. Journal of Biological Chemistry, 2007. 282(49): p. 36112-36120.

84. Wagner, R.J., et al., Lovastatin induces VSMC differentiation through inhibition of Rheb and mTOR. American Journal of Physiology-Cell Physiology, 2010. 299(1): p. C119-C127.

85. Oefner, $\mathrm{C}$., et al., Crystal structure of human platelet-derived growth factor $B B$. EMBO J, 1992. 11(11): p. 3921-6.

86. Zhan, Y., et al., Role of $J N K, p 38$, and ERK in platelet-derived growth factor-induced vascular proliferation, migration, and gene expression. Arterioscler Thromb Vasc Biol, 2003. 23(5): p. 795-801.

87. Proctor, B.M., et al., Requirement for p38 mitogen-activated protein kinase activity in neointima formation after vascular injury. Circulation, 2008. 118(6): p. 658-66.

88. Miano, J.M., Serum response factor: toggling between disparate programs of gene expression. J Mol Cell Cardiol, 2003. 35(6): p. 577-93.

89. Sun, Q., et al., Defining the mammalian CArGome. Genome Res, 2006. 16(2): p. 197-207.

90. Wang, D.Z. and E.N. Olson, Control of smooth muscle development by the myocardin family of transcriptional coactivators. Curr Opin Genet Dev, 2004. 14(5): p. 558-66. 
91. Wang, Z., et al., Myocardin and ternary complex factors compete for SRF to control smooth muscle gene expression. Nature, 2004. 428(6979): p. 185-9.

92. Yoshida, T., Q. Gan, and G.K. Owens, Kruppel-like factor 4, Elk-1, and histone deacetylases cooperatively suppress smooth muscle cell differentiation markers in response to oxidized phospholipids. Am J Physiol Cell Physiol, 2008. 295(5): p. C1175-82.

93. Deaton, R.A., Q. Gan, and G.K. Owens, Sp1-dependent activation of KLF4 is required for PDGF-BB-induced phenotypic modulation of smooth muscle. Am J Physiol Heart Circ Physiol, 2009. 296(4): p. H1027-37.

94. Zheng, B., M. Han, and J.K. Wen, Role of Kruppel-like factor 4 in phenotypic switching and proliferation of vascular smooth muscle cells. IUBMB Life, 2010. 62(2): p. 132-9.

95. Yoshida, T., K.H. Kaestner, and G.K. Owens, Conditional deletion of Kruppel-like factor 4 delays downregulation of smooth muscle cell differentiation markers but accelerates neointimal formation following vascular injury. Circ Res, 2008. 102(12): p. 1548-57.

96. Liu, Y., et al., Kruppel-like factor 4 abrogates myocardin-induced activation of smooth muscle gene expression. J Biol Chem, 2005. 280(10): p. 9719-27.

97. McDonald, O.G. and G.K. Owens, Programming smooth muscle plasticity with chromatin dynamics. Circulation Research, 2007. 100(10): p. 1428-1441.

98. McDonald, O.G. and G.K. Owens, Programming smooth muscle plasticity with chromatin dynamics. Circ Res, 2007. 100(10): p. 1428-41.

99. McDonald, O.G., et al., Control of SRF binding to CArG box chromatin regulates smooth muscle gene expression in vivo. J Clin Invest, 2006. 116(1): p. 36-48.

100. Sluijter, J.P., D.P. de Kleijn, and G. Pasterkamp, Vascular remodeling and protease inhibition--bench to bedside. Cardiovasc Res, 2006. 69(3): p. 595-603.

101. Wang, L., et al., ADAMTS-7 mediates vascular smooth muscle cell migration and neointima formation in balloon-injured rat arteries. Circ Res, 2009. 104(5): p. 688-98.

102. Wang, L., et al., Cartilage oligomeric matrix protein maintains the contractile phenotype of vascular smooth muscle cells by interacting with alpha(7)beta(1) integrin. Circ Res, 2010. 106(3): p. 514-25.

103. Wang, X., et al., Osteopontin expression in platelet-derived growth factor-stimulated vascular smooth muscle cells and carotid artery after balloon angioplasty. Arterioscler Thromb Vasc Biol, 1996. 16(11): p. 1365-72.

104. O'Brien, E.R., et al., Osteopontin is synthesized by macrophage, smooth muscle, and endothelial cells in primary and restenotic human coronary atherosclerotic plaques. Arterioscler Thromb, 1994. 14(10): p. 1648-56.

105. Giachelli, C.M., et al., Osteopontin is elevated during neointima formation in rat arteries and is a novel component of human atherosclerotic plaques. J Clin Invest, 1993. 92(4): p. 1686-96.

106. Panda, D., et al., Potential roles of osteopontin and alphaVbeta3 integrin in the development of coronary artery restenosis after angioplasty. Proc Natl Acad Sci U S A, 1997. 94(17): p. 9308-13.

107. Matsui, Y., et al., Osteopontin deficiency attenuates atherosclerosis in female apolipoprotein E-deficient mice. Arterioscler Thromb Vasc Biol, 2003. 23(6): p. 1029-34.

108. Leali, D., et al., Osteopontin overexpression inhibits in vitro re-endothelialization via integrin engagement. J Biol Chem, 2007. 282(27): p. 19676-84.

109. Zheng, Y.H., et al., Osteopontin stimulates autophagy via integrin/CD44 and p38 MAPK signaling pathways in vascular smooth muscle cells. J Cell Physiol, 2012. 227(1): p. 12735. 
110. Zheng, Y.H., et al., Osteopontin Stimulates Autophagy Via Integrin/CD44 and p38 MAPK Signaling Pathways in Vascular Smooth Muscle Cells. Journal of Cellular Physiology, 2012. 227(1): p. 127-135.

111. Lehto, S., et al., Medial artery calcification. A neglected harbinger of cardiovascular complications in non-insulin-dependent diabetes mellitus. Arterioscler Thromb Vasc Biol, 1996. 16(8): p. 978-83.

112. Niskanen, L.K., et al., Aortic and lower limb artery calcification in type 2 (non-insulindependent) diabetic patients and non-diabetic control subjects. A five year follow-up study. Atherosclerosis, 1990. 84(1): p. 61-71.

113. Bidder, M., et al., Osteopontin transcription in aortic vascular smooth muscle cells is controlled by glucose-regulated upstream stimulatory factor and activator protein-1 activities. J Biol Chem, 2002. 277(46): p. 44485-96.

114. Nilsson-Berglund, L.M., et al., Nuclear factor of activated T cells regulates osteopontin expression in arterial smooth muscle in response to diabetes-induced hyperglycemia. Arterioscler Thromb Vasc Biol, 2010. 30(2): p. 218-24.

115. Aikawa, M., et al., Redifferentiation of smooth muscle cells after coronary angioplasty determined via myosin heavy chain expression. Circulation, 1997. 96(1): p. 82-90.

116. Aikawa, M., et al., Human Smooth-Muscle Myosin Heavy-Chain Isoforms as Molecular Markers for Vascular Development and Atherosclerosis. Circulation Research, 1993. 73(6): p. 1000-1012.

117. Aikawa, M., et al., Lipid lowering promotes accumulation of mature smooth muscle cells expressing smooth muscle myosin heavy chain isoforms in rabbit atheroma. Circulation Research, 1998. 83(10): p. 1015-1026.

118. Gordon, D., et al., Cell-Proliferation in Human Coronary-Arteries. Proceedings of the National Academy of Sciences of the United States of America, 1990. 87(12): p. 46004604.

119. Kocher, O., et al., Phenotypic Features of Smooth-Muscle Cells during the Evolution of Experimental Carotid-Artery Intimal Thickening - Biochemical and MorphologicalStudies. Laboratory Investigation, 1991. 65(4): p. 459-470.

120. Mosse, P.R., G.R. Campbell, and J.H. Campbell, Smooth muscle phenotypic expression in human carotid arteries. II. Atherosclerosis-free diffuse intimal thickenings compared with the media. Arteriosclerosis, 1986. 6(6): p. 664-9.

121. Yamasaki, Y., et al., Weekly dosing with the platelet-derived growth factor receptor tyrosine kinase inhibitor SU9518 significantly inhibits arterial stenosis. Circulation Research, 2001. 88(6): p. 630-636.

122. Kozaki, K., et al., Blockade of platelet-derived growth factor or its receptors transiently delays but does not prevent fibrous cap formation in ApoE null mice. American Journal of Pathology, 2002. 161(4): p. 1395-1407.

123. Wamhoff, B.R., et al., A G/C element mediates repression of the SM22 alpha promoter within phenotypically modulated smooth muscle cells in experimental atherosclerosis. Circulation Research, 2004. 95(10): p. 981-988.

124. Yoshida, T., K.H. Kaestner, and G.K. Owens, Conditional deletion of Kruppel-like factor 4 delays downregulation of smooth muscle cell differentiation markers but accelerates neointimal formation following vascular injury. Circulation Research, 2008. 102(12): $p$. 1548-1557.

125. Liu, Y., et al., Kruppel-like factor 4 abrogates myocardin-induced activation of smooth muscle gene expression. Journal of Biological Chemistry, 2005. 280(10): p. 9719-9727. 
126. Glickman, M.H. and A. Ciechanover, The ubiquitin-proteasome proteolytic pathway: destruction for the sake of construction. Physiol Rev, 2002. 82(2): p. 373-428.

127. Ciechanover, A., The ubiquitin-proteasome proteolytic pathway. Cell, 1994. 79(1): p. 1321.

128. Mehrpour, M., et al., Autophagy in health and disease. 1. Regulation and significance of autophagy: an overview. Am J Physiol Cell Physiol, 2010. 298(4): p. C776-85.

129. Barringhaus, K.G. and M.E. Matsumura, The proteasome inhibitor lactacystin attenuates growth and migration of vascular smooth muscle cells and limits the response to arterial injury. Exp Clin Cardiol, 2007. 12(3): p. 119-24.

130. Meiners, S., et al., Ubiquitin-proteasome pathway as a new target for the prevention of restenosis. Circulation, 2002. 105(4): p. 483-489.

131. Liao, X., et al., Macrophage autophagy plays a protective role in advanced atherosclerosis. Cell Metab, 2012. 15(4): p. 545-53.

132. Razani, B., et al., Autophagy links inflammasomes to atherosclerotic progression. Cell Metab, 2012. 15(4): p. 534-44.

133. Hill, B.G., et al., Unsaturated lipid peroxidation-derived aldehydes activate autophagy in vascular smooth-muscle cells. Biochem J, 2008. 410(3): p. 525-34.

134. Martinet, W., et al., Interactions between cell death induced by statins and 7ketocholesterol in rabbit aorta smooth muscle cells. British Journal of Pharmacology, 2008. 154(6): p. 1236-1246.

135. Czaja, M.J., Autophagy in health and disease. 2. Regulation of lipid metabolism and storage by autophagy: pathophysiological implications. Am J Physiol Cell Physiol, 2010. 298(5): p. C973-8.

136. Heiden, M.G.V., L.C. Cantley, and C.B. Thompson, Understanding the Warburg Effect: The Metabolic Requirements of Cell Proliferation. Science, 2009. 324(5930): p. 10291033.

137. Moncada, S., E.A. Higgs, and S.L. Colombo, Fulfilling the metabolic requirements for cell proliferation. Biochem J, 2012. 446(1): p. 1-7.

138. Vander Heiden, M.G., L.C. Cantley, and C.B. Thompson, Understanding the Warburg effect: the metabolic requirements of cell proliferation. Science, 2009. 324(5930): p. 1029-33.

139. Christofk, H.R., et al., Pyruvate kinase M2 is a phosphotyrosine-binding protein. Nature, 2008. 452(7184): p. 181-U27.

140. DeBerardinis, R.J., et al., The biology of cancer: Metabolic reprogramming fuels cell growth and proliferation. Cell Metabolism, 2008. 7(1): p. 11-20.

141. Tsakiridis, T., et al., Multiple Roles of Phosphatidylinositol 3-Kinase in Regulation of Glucose-Transport, Amino-Acid-Transport, and Glucose Transporters in L6 SkeletalMuscle Cells. Endocrinology, 1995. 136(10): p. 4315-4322.

142. Ranganna, K. and F.M. Yatsu, Inhibition of platelet-derived growth factor BB-induced expression of glyceraldehyde-3-phosphate dehydrogenase by sodium butyrate in rat vascular smooth muscle cells. Arterioscler Thromb Vasc Biol, 1997. 17(12): p. 3420-7.

143. Evans, J.L., et al., The effects of wortmannin, a potent inhibitor of phosphatidylinositol 3kinase, on insulin-stimulated glucose transport, GLUT4 translocation, antilipolysis, and DNA synthesis. Cell Signal, 1995. 7(4): p. 365-76.

144. Werle, M., et al., Metabolic control analysis of the Warburg-effect in proliferating vascular smooth muscle cells. J Biomed Sci, 2005. 12(5): p. 827-34.

145. Perez, J., et al., Role of cellular bioenergetics in smooth muscle cell proliferation induced by platelet-derived growth factor. Biochem J, 2010. 428(2): p. 255-67. 
146. Mack, C.P., Signaling mechanisms that regulate smooth muscle cell differentiation. Arterioscler Thromb Vasc Biol, 2011. 31(7): p. 1495-505.

147. Pyle, A.L. and P.P. Young, Atheromas feel the pressure: biomechanical stress and atherosclerosis. Am J Pathol, 2010. 177(1): p. 4-9.

148. Ferns, G.A., et al., Inhibition of neointimal smooth muscle accumulation after angioplasty by an antibody to PDGF. Science, 1991. 253(5024): p. 1129-32.

149. Jackson, C.L., et al., Role of endogenous platelet-derived growth factor in arterial smooth muscle cell migration after balloon catheter injury. Arterioscler Thromb, 1993. 13(8): p. 1218-26.

150. Lewis, C.D., et al., Modulation of smooth muscle proliferation in rat carotid artery by platelet-derived mediators and fibroblast growth factor-2. Platelets, 2001. 12(6): p. 3528.

151. Giese, N.A., et al., The role of alpha and beta platelet-derived growth factor receptor in the vascular response to injury in nonhuman primates. Arterioscler Thromb Vasc Biol, 1999. 19(4): p. 900-9.

152. Hart, C.E., et al., PDGFbeta receptor blockade inhibits intimal hyperplasia in the baboon. Circulation, 1999. 99(4): p. 564-9.

153. Noiseux, N., et al., Bolus endovascular PDGFR-beta antisense treatment suppressed intimal hyperplasia in a rat carotid injury model. Circulation, 2000. 102(11): p. 1330-6.

154. Sirois, M.G., M. Simons, and E.R. Edelman, Antisense oligonucleotide inhibition of PDGFR-beta receptor subunit expression directs suppression of intimal thickening. Circulation, 1997. 95(3): p. 669-76.

155. Leppanen, O., et al., Intimal hyperplasia recurs after removal of PDGF-AB and $-B B$ inhibition in the rat carotid artery injury model. Arterioscler Thromb Vasc Biol, 2000. 20(11): p. E89-95.

156. Buetow, B.S., et al., Chimera analysis supports a predominant role of PDGFRbeta in promoting smooth-muscle cell chemotaxis after arterial injury. Am J Pathol, 2003. 163(3): p. 979-84.

157. Myllarniemi, M., et al., Inhibition of platelet-derived growth factor receptor tyrosine kinase inhibits vascular smooth muscle cell migration and proliferation. FASEB J, 1997. 11(13): p. 1119-26.

158. Yamasaki, Y., et al., Weekly dosing with the platelet-derived growth factor receptor tyrosine kinase inhibitor SU9518 significantly inhibits arterial stenosis. Circ Res, 2001. 88(6): p. 630-6.

159. Yu, J.C., et al., Efficacy of the novel selective platelet-derived growth factor receptor antagonist CT52923 on cellular proliferation, migration, and suppression of neointima following vascular injury. J Pharmacol Exp Ther, 2001. 298(3): p. 1172-8.

160. Palmberg, L., M. Sjolund, and J. Thyberg, Phenotype modulation in primary cultures of arterial smooth-muscle cells: reorganization of the cytoskeleton and activation of synthetic activities. Differentiation, 1985. 29(3): p. 275-83.

161. Worth, N.F., et al., Vascular smooth muscle cell phenotypic modulation in culture is associated with reorganisation of contractile and cytoskeletal proteins. Cell Motil Cytoskeleton, 2001. 49(3): p. 130-45.

162. Thyberg, J., et al., Phenotype modulation in primary cultures of arterial smooth muscle cells. On the role of platelet-derived growth factor. Differentiation, 1983. 25(2): p. 15667.

163. Perez, J., et al., Role of cellular bioenergetics in smooth muscle cell proliferation induced by platelet-derived growth factor. Biochemical Journal, 2010. 428(2): p. 255-67. 
164. Thyberg, J. and K. Blomgren, Effects of proteasome and calpain inhibitors on the structural reorganization and proliferation of vascular smooth muscle cells in primary culture. Lab Invest, 1999. 79(9): p. 1077-88.

165. Sandbo, N., et al., Regulation of serum response factor-dependent gene expression by proteasome inhibitors. Mol Pharmacol, 2005. 67(3): p. 789-97.

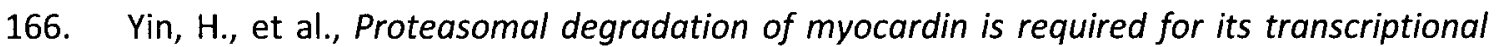
activity in vascular smooth muscle cells. J Cell Physiol, 2011. 226(7): p. 1897-906.

167. Codogno, P. and A.J. Meijer, Autophagy and signaling: their role in cell survival and cell death. Cell Death and Differentiation, 2005. 12: p. 1509-1518.

168. Klionsky, D.J., et al., Guidelines for the use and interpretation of assays for monitoring autophagy in higher eukaryotes. Autophagy, 2008. 4(2): p. 151-175.

169. Klionsky, D.J., et al., Does bafilomycin A1 block the fusion of autophagosomes with lysosomes? Autophagy, 2008. 4(7): p. 849-950.

170. Lindner, V., et al., Role of basic fibroblast growth factor in vascular lesion formation. Circ Res, 1991. 68(1): p. 106-13.

171. Seglen, P.O. and P.B. Gordon, 3-Methyladenine: specific inhibitor of autophagic/lysosomal protein degradation in isolated rat hepatocytes. Proc Natl Acad Sci U S A, 1982. 79(6): p. 1889-92.

172. Xue, L.Z., G.C. Fletcher, and A.M. Tolkovsky, Autophagy is activated by apoptotic signalling in sympathetic neurons: An alternative mechanism of death execution. Molecular and Cellular Neuroscience, 1999. 14(3): p. 180-198.

173. Blommaart, E.F.C., et al., The phosphatidylinositol 3-kinase inhibitors wortmannin and LY294002 inhibit autophagy in isolated rat hepatocytes. European Journal of Biochemistry, 1997. 243(1-2): p. 240-246.

174. Ito, S., et al., 3-methyladenine suppresses cell migration and invasion of HT1080 fibrosarcoma cells through inhibiting phosphoinositide 3-kinases independently of autophagy inhibition. International Journal of Oncology, 2007. 31(2): p. 261-268.

175. Liu, J.L., et al., Beclin1 Controls the Levels of $p 53$ by Regulating the Deubiquitination Activity of USP10 and USP13. Cell, 2011. 147(1): p. 223-234.

176. Meng, L., et al., Epoxomicin, a potent and selective proteasome inhibitor, exhibits in vivo antiinflammatory activity. Proc Natl Acad Sci U S A, 1999. 96(18): p. 10403-8.

177. Jurgens, G., et al., Immunostaining of human autopsy aortas with antibodies to modified apolipoprotein B and apoprotein(a). Arterioscler Thromb, 1993. 13(11): p. 1689-99.

178. Palinski, W., et al., ApoE-deficient mice are a model of lipoprotein oxidation in atherogenesis. Demonstration of oxidation-specific epitopes in lesions and high titers of autoantibodies to malondialdehyde-lysine in serum. Arterioscler Thromb, 1994. 14(4): p. 605-16.

179. Higdon, A.N., et al., Hemin causes mitochondrial dysfunction in endothelial cells through promoting lipid peroxidation: the protective role of autophagy. Am J Physiol Heart Circ Physiol, 2012. 302(7): p. H1394-409.

180. Garat, C., et al., Induction of smooth muscle alpha-actin in vascular smooth muscle cells by arginine vasopressin is mediated by c-Jun amino-terminal kinases and p38 mitogenactivated protein kinase. Journal of Biological Chemistry, 2000. 275(29): p. 22537-22543.

181. Hautmann, M.B., et al., Angiotensin II-induced stimulation of smooth muscle alpha-actin expression by serum response factor and the homeodomain transcription factor MHox. Circulation Research, 1997. 81(4): p. 600-610. 
182. Higashita, R., et al., Galpha16 mimics vasoconstrictor action to induce smooth muscle alpha-actin in vascular smooth muscle cells through a Jun-NH2-terminal kinasedependent pathway. Journal of Biological Chemistry, 1997. 272(41): p. 25845-50.

183. Thyberg, J. and A. Hultgardhnilsson, Fibronectin and the Basement-Membrane Components Laminin and Collagen Type-Iv Influence the Phenotypic Properties of Subcultured Rat Aortic Smooth-Muscle Cells Differently. Cell and Tissue Research, 1994. 276(2): p. 263-271.

184. Su, B., et al., Redox regulation of vascular smooth muscle cell differentiation. Circulation Research, 2001. 89(1): p. 39-46.

185. Wang, L., et al., ADAMTS-7 Mediates Vascular Smooth Muscle Cell Migration and Neointima Formation in Balloon-Injured Rat Arteries. Circulation Research, 2009. 104(5): p. 688-U247.

186. Yoshida, T. and G.K. Owens, Molecular determinants of vascular smooth muscle cell diversity. Circulation Research, 2005. 96(3): p. 280-91.

187. Davis, B.N., et al., Induction of MicroRNA-221 by Platelet-derived Growth Factor Signaling is Critical for Modulation of Vascular Smooth Muscle Phenotype. Journal of Biological Chemistry, 2009. 284(6): p. 3728-3738.

188. Liu, X.J., et al., A Necessary Role of miR-221 and miR-222 in Vascular Smooth Muscle Cell Proliferation and Neointimal Hyperplasia. Circulation Research, 2009. 104(4): p. 476U125.

189. Demasi, M. and F.R. Laurindo, Physiological and pathological role of the ubiquitinproteasome system in the vascular smooth muscle cell. Cardiovasc Res, 2012.

190. Jia, G., et al., Insulin-like growth factor-1 and TNF-alpha regulate autophagy through cjun N-terminal kinase and Akt pathways in human atherosclerotic vascular smooth cells. Immunol Cell Biol, 2006. 84(5): p. 448-54.

191. Capparelli, C., et al., CTGF drives autophagy, glycolysis and senescence in cancerassociated fibroblasts via HIF1 activation, metabolically promoting tumor growth. Cell Cycle, 2012. 11(12): p. 2272-84.

192. Kiyono, K., et al., Autophagy is activated by TGF-beta and potentiates TGF-betamediated growth inhibition in human hepatocellular carcinoma cells. Cancer Res, 2009. 69(23): p. 8844-52.

193. Ding, M., et al., Adiponectin Induces Vascular Smooth Muscle Cell Differentiation via Repression of Mammalian Target of Rapamycin Complex 1 and FoxO4. Arteriosclerosis Thrombosis and Vascular Biology, 2011. 31(6): p. 1403-U366.

194. Tannous, P., et al., Intracellular protein aggregation is a proximal trigger of cardiomyocyte autophagy. Circulation, 2008. 117(24): p. 3070-8.

195. Martinet, W. and G.R. De Meyer, Autophagy in atherosclerosis: a cell survival and death phenomenon with therapeutic potential. Circ Res, 2009. 104(3): p. 304-17.

196. Tammali, R., et al., Aldose Reductase Regulates Vascular Smooth Muscle Cell Proliferation by Modulating G1/S Phase Transition of Cell Cycle. Endocrinology, 2010. 151(5): p. 2140-2150.

197. Xing, D.Q., et al., Increased protein O-GlCNAC modification inhibits inflammatory and neointimal responses to acute endoluminal arterial injury. American Journal of Physiology-Heart and Circulatory Physiology, 2008. 295(1): p. H335-H342.

198. Perez, J., et al., Role of cellular bioenergetics in smooth muscle cell proliferation induced by platelet-derived growth factor. Biochemical Journal, 2010. 428: p. 255-267.

199. Gupte, R.S., et al., Glucose-6-Phosphate Dehydrogenase is a Regulator of Vascular Smooth Muscle Contraction. Antioxidants \& Redox Signaling, 2011. 14(4): p. 543-558. 
200. Roger, Heart Disease and Stroke Statistics-2011 Update: A Report From the American Heart Association (vol 123, pg e18, 2011). Circulation, 2011. 124(16): p. E426-E426.

201. Bidder, M., et al., Osteopontin transcription in aortic vascular smooth muscle cells is controlled by glucose-regulated upstream stimulatory factor and activator protein-1 activities. Journal of Biological Chemistry, 2002. 277(46): p. 44485-44496.

202. Marshall, S., V. Bacote, and R.R. Traxinger, Discovery of a metabolic pathway mediating glucose-induced desensitization of the glucose transport system. Role of hexosamine biosynthesis in the induction of insulin resistance. J Biol Chem, 1991. 266(8): p. 4706-12.

203. Xing, D., et al., Increased protein O-GICNAC modification inhibits inflammatory and neointimal responses to acute endoluminal arterial injury. Am J Physiol Heart Circ Physiol, 2008. 295(1): p. H335-42.

204. Laczy, B., et al., Protein O-GlcNAcylation: a new signaling paradigm for the cardiovascular system. Am J Physiol Heart Circ Physiol, 2009. 296(1): p. H13-28.

205. Lehle, L., S. Strahl, and W. Tanner, Protein glycosylation, conserved from yeast to man: $a$ model organism helps elucidate congenital human diseases. Angew Chem Int Ed Engl, 2006. 45(41): p. 6802-18.

206. Guinez, C., et al., O-GICNAC glycosylation: a signal for the nuclear transport of cytosolic proteins? Int J Biochem Cell Biol, 2005. 37(4): p. 765-74.

207. Comer, F.I. and G.W. Hart, O-GICNAC and the control of gene expression. Biochim Biophys Acta, 1999. 1473(1): p. 161-71.

208. Liu, K., et al., Accumulation of protein O-GlcNAC modification inhibits proteasomes in the brain and coincides with neuronal apoptosis in brain areas with high O-GICNAC metabolism. J Neurochem, 2004. 89(4): p. 1044-55.

209. Zachara, N.E. and G.W. Hart, O-GICNAC a sensor of cellular state: the role of nucleocytoplasmic glycosylation in modulating cellular function in response to nutrition and stress. Biochim Biophys Acta, 2004. 1673(1-2): p. 13-28.

210. Zachara, N.E. and G.W. Hart, Cell signaling, the essential role of O-GICNAC! Biochim Biophys Acta, 2006. 1761(5-6): p. 599-617.

211. Wells, L. and G.W. Hart, O-GlCNAC turns twenty: functional implications for posttranslational modification of nuclear and cytosolic proteins with a sugar. FEBS Lett, 2003. 546(1): p. 154-8.

212. Vosseller, K., et al., Diverse regulation of protein function by O-GICNAc: a nuclear and cytoplasmic carbohydrate post-translational modification. Curr Opin Chem Biol, 2002. 6(6): p. 851-7.

213. Guinez, C., et al., Protein ubiquitination is modulated by O-GlcNAc glycosylation. FASEB J, 2008. 22(8): p. 2901-11.

214. Shaw, P., et al., Regulation of specific DNA binding by $p 53$ : evidence for a role for $O$ glycosylation and charged residues at the carboxy-terminus. Oncogene, 1996. 12(4): p. 921-30.

215. Donadio, A.C., et al., Antisense glutaminase inhibition modifies the O-GlcNAc pattern and flux through the hexosamine pathway in breast cancer cells. J Cell Biochem, 2008. 103(3): p. 800-11.

216. Dias, W.B. and G.W. Hart, O-GICNAC modification in diabetes and Alzheimer's disease. Mol Biosyst, 2007. 3(11): p. 766-72.

217. Hanover, J.A., Glycan-dependent signaling: O-linked N-acetylglucosamine. FASEB J, 2001. 15(11): p. 1865-76.

218. Haltiwanger, R.S., G.D. Holt, and G.W. Hart, Enzymatic addition of O-GICNAC to nuclear and cytoplasmic proteins. Identification of a uridine diphospho- $N$ - 
acetylglucosamine:peptide beta-N-acetylglucosaminyltransferase. J Biol Chem, 1990. 265(5): p. 2563-8.

219. Gao, Y., et al., Dynamic O-glycosylation of nuclear and cytosolic proteins: cloning and characterization of a neutral, cytosolic beta- $\mathrm{N}$-acetylglucosaminidase from human brain. J Biol Chem, 2001. 276(13): p. 9838-45.

220. Kreppel, L.K. and G.W. Hart, Regulation of a cytosolic and nuclear O-GlcNAc transferase. Role of the tetratricopeptide repeats. J Biol Chem, 1999. 274(45): p. 32015-22.

221. Nerlich, A.G., et al., Expression of glutamine:fructose-6-phosphate amidotransferase in human tissues: evidence for high variability and distinct regulation in diabetes. Diabetes, 1998. 47(2): p. 170-8.

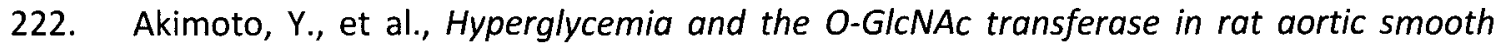
muscle cells: elevated expression and altered patterns of O-GlcNAcylation. Arch Biochem Biophys, 2001. 389(2): p. 166-75.

223. Slawson, $\mathrm{C}$., et al., Perturbations in O-linked beta-N-acetylglucosamine protein modification cause severe defects in mitotic progression and cytokinesis. J Biol Chem, 2005. 280(38): p. 32944-56.

224. Endo, A., et al., Specific inhibition of glyceraldehyde-3-phosphate dehydrogenase by koningic acid (heptelidic acid). J Antibiot (Tokyo), 1985. 38(7): p. 920-5.

225. Yuzwa, S.A., et al., A potent mechanism-inspired O-GlcNAcase inhibitor that blocks phosphorylation of tau in vivo. Nature Chemical Biology, 2008. 4(8): p. 483-490.

226. Ngoh, G.A., et al., O-GIcNAC signaling in the cardiovascular system. Circ Res, 2010. 107(2): p. 171-85.

227. Jalvy, S., et al., Autocrine expression of osteopontin contributes to PDGF-mediated arterial smooth muscle cell migration. Cardiovasc Res, 2007. 75(4): p. 738-47.

228. Renault, M.A., et al., AP-1 is involved in UTP-induced osteopontin expression in arterial smooth muscle cells. Circulation Research, 2003. 93(7): p. 674-681.

229. Denhardt, D.T., et al., Transcriptional regulation of osteopontin and the metastatic phenotype: evidence for a Ras-activated enhancer in the human OPN promoter. Clin Exp Metastasis, 2003. 20(1): p. 77-84.

230. Srivastava, S., et al., Contribution of aldose reductase to diabetic hyperproliferation of vascular smooth muscle cells. Diabetes, 2006. 55(4): p. 901-10.

231. Bhatnagar, A., et al., Regulation of vascular smooth muscle cell growth by aldose reductase. Chem Biol Interact, 2001. 130-132(1-3): p. 627-36.

232. Park, M.K., et al., Perinuclear, perigranular and sub-plasmalemmal mitochondria have distinct functions in the regulation of cellular calcium transport. Embo Journal, 2001. 20(8): p. 1863-1874.

233. Youle, R.J. and M. Karbowski, Mitochondrial fission in apoptosis. Nat Rev Mol Cell Biol, 2005. 6(8): p. 657-63.

234. Mitra, K., et al., A hyperfused mitochondrial state achieved at $G(1)-S$ regulates cyclin $E$ buildup and entry into $S$ phase. Proceedings of the National Academy of Sciences of the United States of America, 2009. 106(29): p. 11960-11965.

235. Smirnova, E., et al., A human dynamin-related protein controls the distribution of mitochondria. Molecular Biology of the Cell, 1998. 9: p. 197a-197a.

236. Yoon, Y., et al., The mitochondrial protein hFis1 regulates mitochondrial fission in mammalian cells through an interaction with the dynamin-like protein DLP1. Molecular and Cellular Biology, 2003. 23(15): p. 5409-5420. 
237. Martinou, I., et al., The release of cytochrome $c$ from mitochondria during apoptosis of NGF-deprived sympathetic neurons is a reversible event. Journal of Cell Biology, 1999. 144(5): p. 883-889.

238. Frank, S., et al., The role of dynamin-related protein 1, a mediator of mitochondrial fission, in apoptosis. Developmental Cell, 2001. 1(4): p. 515-525.

239. Rambold, A.S., et al., Tubular network formation protects mitochondria from autophagosomal degradation during nutrient starvation. Proc Natl Acad Sci U S A, 2011. 108(25): p. 10190-5.

240. Brady, N.R., A. Hamacher-Brady, and R.A. Gottlieb, Proapoptotic BCL-2 family members and mitochondrial dysfunction during ischemia/reperfusion injury, a study employing cardiac HL-1 cells and GFP biosensors. Biochimica Et Biophysica Acta-Bioenergetics, 2006. 1757(5-6): p. 667-678.

241. Makino, A., B.T. Scott, and W.H. Dillmann, Mitochondrial fragmentation and superoxide anion production in coronary endothelial cells from a mouse model of type 1 diabetes. Diabetologia, 2010. 53(8): p. 1783-1794.

242. Hom, J., et al., Regulation of mitochondrial fission by intracellular $\mathrm{Ca} 2+$ in rat ventricular myocytes. Biochim Biophys Acta, 2010. 1797(6-7): p. 913-21.

243. Chen, L., et al., Mitochondrial OPA1, apoptosis, and heart failure. Cardiovasc Res, 2009. 84(1): p. 91-9.

244. Chen, K.H., et al., Dysregulation of HSG triggers vascular proliferative disorders. Nat Cell Biol, 2004. 6(9): p. 872-83.

245. Marsboom, G., et al., Dynamin-related protein 1-mediated mitochondrial mitotic fission permits hyperproliferation of vascular smooth muscle cells and offers a novel therapeutic target in pulmonary hypertension. Circulation Research, 2012. 110(11): p. 1484-97.

246. Schreurs, M., F. Kuipers, and F.R. van der Leij, Regulatory enzymes of mitochondrial beta-oxidation as targets for treatment of the metabolic syndrome. Obesity Reviews, 2010. 11(5): p. 380-388.

247. Marsboom, G., et al., Dynamin-Related Protein 1-Mediated Mitochondrial Mitotic Fission Permits Hyperproliferation of Vascular Smooth Muscle Cells and Offers a Novel Therapeutic Target in Pulmonary Hypertension. Circulation Research, 2012. 110(11): p. 1484-+.

248. Rambold, A.S., et al., Tubular network formation protects mitochondria from autophagosomal degradation during nutrient starvation. Proceedings of the National Academy of Sciences of the United States of America, 2011. 108(25): p. 10190-10195.

249. Cassidy-Stone, A., et al., Chemical inhibition of the mitochondrial division dynamin reveals its role in Bax/Bak-dependent mitochondrial outer membrane permeabilization. Dev Cell, 2008. 14(2): p. 193-204.

250. Houten, S.M. and R.J. Wanders, A general introduction to the biochemistry of mitochondrial fatty acid beta-oxidation. J Inherit Metab Dis, 2010. 33(5): p. 469-77.

251. Bartlett, K. and S. Eaton, Mitochondrial beta-oxidation. European Journal of Biochemistry, 2004. 271(3): p. 462-469.

252. Lopez-Vinas, E., et al., Definition by functional and structural analysis of two malonylCoA sites in carnitine palmitoy/transferase 1A. J Biol Chem, 2007. 282(25): p. 18212-24.

253. Kerner, J. and C. Hoppel, Fatty acid import into mitochondria. Biochimica Et Biophysica Acta-Molecular and Cell Biology of Lipids, 2000. 1486(1): p. 1-17.

254. Bach, D., et al., Mitofusin-2 determines mitochondrial network architecture and mitochondrial metabolism. A novel regulatory mechanism altered in obesity. Journal of Biological Chemistry, 2003. 278(19): p. 17190-7. 
255. Debnath, J., E.H. Baehrecke, and G. Kroemer, Does autophagy contribute to cell death? Autophagy, 2005. 1(2): p. 66-74.

256. Guo, Y.H., et al., Overexpression of Mitofusin 2 inhibited oxidized low-density lipoprotein induced vascular smooth muscle cell proliferation and reduced atherosclerotic lesion formation in rabbit. Biochem Biophys Res Commun, 2007. 363(2): p. 411-7. 


\title{
APPENDIX
}

\section{NON-STANDARD ABBREVIATIONS AND ACRONYMS}

\author{
VSMCs Vascular smooth muscle cells \\ SP-1 Spautin 1 \\ 3-MA 3-methyl adenine \\ USP Ubiquitin specific peptidase \\ PDGF Platelet-derived growth factor \\ HBP Hexosamine biosynthetic pathway \\ GFAT Glutamine: Fructose amidotransferase \\ UDP-GICNAc Uridine diphospho- $\beta$-N-acetylglucosamine \\ O-GIcNAC $\quad \beta$-O-linked $\mathrm{N}$-acetylglucosamine \\ Ad-GFP Adenovirus-delivered green fluorescent protein \\ Ad-GFP-LC3 Adenovirus-delivered green fluorescent protein linked LC3 \\ PUGNAC O-(2-acetamido-2-deoxy-d-glycopyranosylidene) amino-N- \\ phenylcarbamate \\ OGT O-GIcNAc transferase \\ LDH Lactate dehydrogenase \\ MTT (3-(4,5-Dimethylthiazol-2-yl)-2,5-diphenyltetrazolium bromide) \\ PCNA Proliferating cell nuclear antigen
}


Mfn2 Mitofusin 2

Mdivi-1 Mitochondria division inhibitor 1

ECM Extracellular matrix

OPN Osteopontin

FGF-1 Fibroblast growth factor 1

LC3 Microtubule-associated protein light chain 3

FAO Fatty acid oxidation

ECAR Extracellular acidification rate

OCR Oxygen consumption rate

HNE 4-hydroxy-trans-2-nonenal

CPT1 Carnitine palmitoyl transferase 1

LDL Low density lipoprotein

TCA Tricarboxylic acid cycle

KA Koningic acid

MTC01 Mitochondrially encoded cytochrome $\mathrm{c}$ oxidase I

SDHB Succinate dehydrogenase [ubiquinone] iron-sulfur subunit

ATP5A $\quad$ ATP synthase subunit alpha

NDFUB8 NADH dehydrogenase (ubiquinone) 1 beta subcomplex subunit 8 


\title{
CURRICULLUM VITAE
}

\author{
Joshua K Salabei, MS \\ Graduate student, Department of Biochemistry and Molecular Biology and the \\ Diabetes and Obesity Center, \\ University of Louisville School of Medicine, \\ 580 S Preston Street, Room 407, Louisville, KY40202 \\ Office: 502-852-1029, Cell: 502-235-6407 \\ Email:kisala02@louisville.edu
}

\section{EDUCATION}

\section{Graduation and Post-Graduation}

Year of Degree

2004
Degree

BS, Biochemistry and

Medical Lab Technology

Institution 
University of Nottingham, Technology

Anticipated degree date 2012

Ph.D. Biochemistry and Molecular Biology

University of Louisville, Kentucky

\section{Positions:}

$05 / 25 / 07-08 / 31 / 07$

Student Research Assistant, Dan Plamer, Ph.D, Q-Chip Ltd, Cardiff medicentre, Heath Park, Cardiff, UK

30/09/07-01/13/08 Student Research Assistant, Chuan Hu, Ph.D, Assistant Professor, Department of Biochemistry and Molecular Biology, University of Louisville, Louisville, KY

01/19/08-06/19/08

Student Research Assistant, Oleg Barski, Ph.D, Assistant Professor of medicine, Department of Medicine-Division of Cardiology, Department of Biochemistry and Molecular Biology, University of Louisville, Louisville, KY

06/19/08-Current

Graduate Student, Advisors: 1). Aruni Bhatnagar, Ph.D, Professor of Medicine, Department of Medicine, Department of Biochemistry and Molecular Biology, University of Louisville, Louisville, KY. 2). Bradford Hill, Ph.D, Assistant Professor of Medicine, Department of Medicine, Department of Biochemistry and Molecular Biology, University of Louisville, Louisville, KY. 


\section{Major research interest:}

Vascular biology and diseases affecting the vasculature

\section{Member society:}

American Society of Biochemistry and Molecular Biology (ASBMB) and American heart association (AHA)

\section{Awards and Honors:}

2011

2010

2009

2007

2006

2004

Seahorse $\$ 1000$ travel award for presentation in EB 2011 scientific conference, Washington DC, USA

$\$ 700$ award at the $15^{\text {th }}$ International Meeting on Enzymology and Molecular Biology of Carbonyl Metabolism. July 6-11, 2010. Lexington, Kentucky, USA

University of Louisville, Department of Biochemistry retreat faculty selected poster presentation award.

2007 Integrated Program for Biomedical Science Research Fellowship (IPIBS), University of Louisville, Kentucky

Developing Solution Taught Masters Fellowship .Grant Amount; $£ 6,250$, University of Nottingham, UK

- Best Biochemistry Student, graduating class of 2004 by Les Brasseries du Cameroon.

- Second overall best student, graduating class of 2004, University of Buea, First class degree, GPA 3.61/4

Financial Aid for outstanding performance by Ministry for Higher Education, Cameroon.

2002 Financial Aid for outstanding performance by Ministry for Higher Education, Cameroon. 
Scientific conferences attended:

2012

Poster presentation, AHA, Los Angeles convention center, LA, CA

2011

Poster presentation, Experimental Biology, Washington DC convention center, Washington DC.

2010

Poster presentation, Research Louisville, Louisville, KY.

2010

2010 Poster presentation, Experimental Biology, Anaheim convention

Poster presentation, $15^{\text {th }}$ International Meeting on Enzymology and Molecular Biology of Carbonyl Metabolism, Lexington, KY. center, Anaheim, California.

2009

Poster presentation, Research Louisville, Louisville, KY.

2007

Applied Biosystem training conference on quantitative real time PCR, Cardiff, United Kingdom.

\section{Publications and Abstracts:}

\section{A. Publication and manuscripts:}

1. Salabei JK, A. Balakumaran, J.C. Frey, P.J. Boor, M. Treinen-Moslen and D.J. Conklin. Verapamil stereoisomers induce antiproliferative effects in vascular smooth muscle cells via autophagy. Toxicol Appl Pharmacol. 2012 Aug 1;262(3):265-72.

2. Salabei JK, Li XP, Petrash JM, Bhatnagar A, Barski OA. Functional expression of novel human and murine AKR1B genes. Chem Biol Interact. 2011 May 30; 191(1-3):177-84.

3. Sansbury BE, Riggs DW, Brainard RE, Salabei JK, Jones SP, Hill BG. Responses of hypertrophied myocytes to reactive species: implications for glycolysis and electrophile metabolism. Biochem J. 2011 Apr 15; 435(2):519-28. 
4. Timothy D. Cummins, Ashlee N. Higdon, Philip A. Kramer Balu Chacko, Daniel W. Riggs, Salabei JK, Victor M. Darley-Usmar, and Hill BG. Utilization of fluorescent probes to identify subcellular proteomes and processes regulated by lipid peroxidation products. Free Radic Biol Med. 2012 Aug 23.

5. Salabei JK, Cummins TD, Bhatnagar A, and Hill BG. PDGF-mediated autophagy regulates vascular smooth muscle cell phenotype transition and resistance to oxidative stress. Manuscript under review in Biochemical Journal

6. Salabei JK, Ivanova M, Lei Z, McCoy T, Li XP, Petrash JM, Bhatnagar A, Barski OA. AKR1B15 is expressed in human tissues. Manuscript in preparation

7. Salabei JK and Conklin DJ. Drug-induced autophagy: Potential applications in cardiovascular diseases. Invited review for Cardiovascular Toxicology (due Sept. 2012).

8. Salabei JK and Hill BG. Implications of autophagy for the smooth muscle cell. Invited review for FRBM (due Dec. 2012)

9. Salabei JK, Cummins TD, Jones SP, Bhatnagar A and Hill BG. Antiproliferative Effects of O-GICNAC on PDGF-Induced Vascular Smooth Muscle Cell Proliferation. Manuscript in preparation for submission to JPET.

10. Salabei JK, Cummins TD, Bhatnagar A and Hill BG. Mitochondrial fragmentation and fatty acid oxidation regulate VSMC proliferation. Manuscript in preparation

\section{B. Abstracts:}

1. Salabei JK, Cummins TD, Bhatnagar A and Bradford G. Hill. PDGFmediated autophagy regulates vascular smooth muscle cell phenotype transition and resistance to oxidative stress. AHA 2012 and Research Louisville 2012

2. Salabei JK, Bhatnagar A, Jones SP and Hill BG. Novel insights into the role of glucose metabolism in PDGF-induced vascular smooth muscle cell phenotypic switching- Research Louisville 2010 and Experimental Biology. 2011.

3. Hill Bradford G.; Haberzettl Petra; Bhatnagar Aruni and Salabei JK. Mitogen-Mediated Autophagy Regulates Vascular Smooth Muscle Cell Phenotype. Free Radic Biol Med Volume: 51 Supplement: 1 page: S41S42. Nov 12011

4. Salabei JK, Magarita Ivanova, Xiao Ping Li, Aruni Bhatnagar and Oleg A. Barski; Cloning, Expression and Characterization of Novel Aldo-Keto 
Reductase 1B (AKR1B) Proteins - Human AKR1B15 and Murine Akr1b10Resarch Louisville 2009 and Experimental Biology 2010.

Oleg A Barski; Xioaping Li; Alan C Brooks; Salabei JK; Aruni Bhatnagar; Srinivas M Tipparaju Redox Regulation of Kv Channels: Obligatory Role of Kv C-terminus in Sensing Pyridine Nucleotide Binding to Kvbeta Subunit American Heart Association, 2009

\section{Technical expertise:}

Western blot, cell culture, qRT-PCR, Immunohistochemistry, RNAi, Adenoviral overexpression, electron microscopy, protein expression and purification, confocal microscopy

\section{Aided in manuscript review for:}

1. Atherosclerosis, Thrombosis and Vascular Biology (ATVB)

2. Chemico-Biological Interactions (CBI)

3. Frontiers in Physiology

\section{References:}

Aruni Bhatnagar, PhD (advisor), Endowed Chair Professor of Medicine, Division of Cardiovascular Medicine, Director of the Diabetes and Obesity Center, University of Louisville School of Medicine, Louisville, KY aruni.bhatnagar@louisville.edu 502-852-5966

Bradford G. Hill, PhD (advisor) Assistant Professor of Medicine, Division of Cardiovascular Medicine, Diabetes and Obesity Center, University of Louisville School of Medicine, Louisville, KY, bradford.hill@louisville.edu 502-852-1015

William L. Dean, PhD

Professor of Biochemistry and Molecular Biology, Vice Chair and Graduate Program Director, University of Louisville School of Medicine, Louisville, KY bill.dean@louisville.edu 502-852-5227 
Russell A. Prough, PhD

Professor of Biochemistry, Associate Vice President for Research and Health Affairs,

Preston Pope Joyes Endowed Chair in Biochemical Research, University of Louisville School of Medicine, Louisville, KY russ.prough@louisville.edu

502-852-7249

Steven P. Jones, PhD

Associate Professor of Medicine, Division of Cardiovascular Medicine, University of Louisville School of Medicine, Louisville, KY, Steven.P.Jones@Louisville.edu

502- 852-246 